\title{
Industrial Waste Management Information for 1990 and Record-to-Date
}

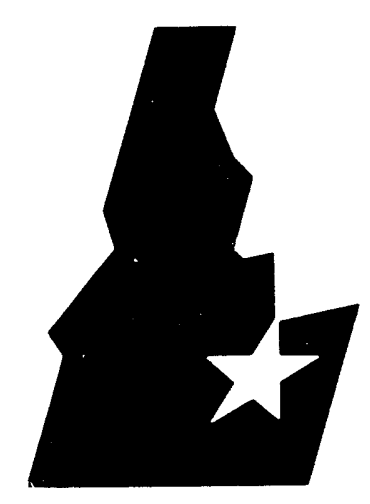

NOY $2=1991$

Idaho National Engineering Laboratory

U.S. Department of Energy Field Office, Idaho

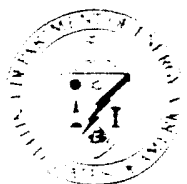

DISTRIBUTION OF THIS DOCUMENT IS UNLIMITEE 


\title{
INDUSTRIAL WASTE MANAGEMENT INFORMATION FOR 1990 AND RECORD-TO-DATE
}

\author{
D. L. Litteer \\ C. N. Peterson \\ A. M. Sims
}

Published August 1991

Idaho National Engineering Laboratory

EG\&G Idaho, Inc.

Idaho Falls, ID 83415

Prepared for the

U.S. Department of Energy

Idaho Operations Office

Under DOE Contract No. DE-AC07-76ID01570

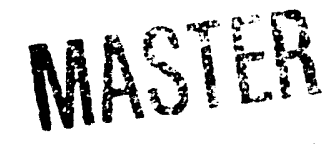

DISTRIBUTION OF THIS DOCURAENT IS UIVLIVITES 


\section{ABSTRACT}

This document provides detailed data and graphics on airborne and liquid effluent releases, fuel oil consumption, and water usage for the calendar year 1990. It summarizes industrial waste data records compiled since 1971 for the Idaho National Engineering Laboratory (INEL). The data presented are from the INEL Industrial Waste Management Information System (IWMIS).

Reports are included on the following:

- INEL Record-to-Date Summary

- INEL 1990 Summaries

- Area Detailed Data Reports for 1990. 


\section{EXECUTIVE SUMMARY}

The Idaho National Engineering Laboratory has used the conputerized database Industrial Waste Management Information System (IWMIS) since 1971. The system serves as the official record for all types of industrial waste stored or disposed of at the INEL. It is also the source for reports on industrial waste storage and disposal, fuel oil usage, and water usage and disposal at the INEL. Quantities of industrial waste are reported for the following categories: airborne and liquid effluent disposal, fuel oil consumption, and water usage.

A total of $80,307 \mathrm{~m}^{3}$ of solid industrial waste were disposed of in sanitary landfills at the INEL during 1990. This represents an increase of $177 \%$ from the $28,956 \mathrm{~m}^{3}$ (revised) discarded in 1989.

During 1990, there were no waste oils and solvents reported.

A total of $2,686,795 \mathrm{~kg}$ of liquid and airborne industrial waste were discharged in 1990 . The industrial waste included $1,713,071 \mathrm{~kg}$ of chemicals, $537,348 \mathrm{~kg}$ of total dissolved solids, and $368,595 \mathrm{~kg}$ of total hardness which were discharged as liquid wastes to the lithosphere at the INEL. This waste represents an $8 \%$ decrease over the total quantity of $2,923,228 \mathrm{~kg}$ in 1989 and a decrease from the total quantity of $3,213,502 \mathrm{~kg}$ in 1988.
The INEL used a total of $8,465,936$ liters of fuel oil during 1990 which produced 105,309 $\mathrm{kg}$ of sulfur dioxide $\left(\mathrm{SO}_{2}\right)$ and $4,754 \mathrm{~kg}$ of particulates. During 1989, 14,658,618 liters of fuel oil were used, producing $217,557 \mathrm{~kg}$ of $\mathrm{SO}_{2}$ and $9,792 \mathrm{~kg}$ of particulates.

A total of 7,539,155,000 liters of water were used by the INEL during 1990 compared to $6,268,060,000$ liters in 1989 and 7,424,356,000 liters in 1988 . However, the majority of this water returned to the lithosphere through seepage ponds. Remaining water was lost to the atmosphere through cooling towers and other evaporatory processes.

NOTE: Argonne National Laboratory - West (ANL) did not operate its cooling towers in April as a result of routine reactor maintenance. In addition, the increase of reported waste at the Sanitary Landfill is a result of a change in reporting methods. Prior to 1990 the waste was reported as compacted waste at the Landfill. During 1990 the waste was reported as uncompacted waste at the Landfill.

Tables and figures found within the tab sections "INEL Record-to-Date Summaries" and "INEL 1990 Summaries" prc ide a detailed overview of INEL industrial waste management data. 


\section{CONTENTS}

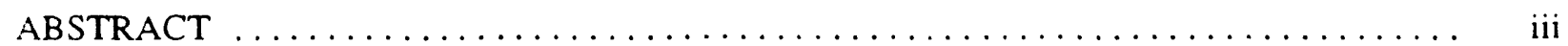

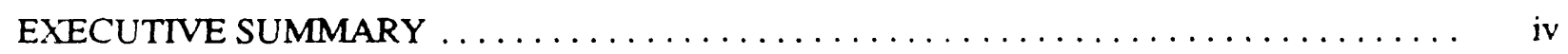

ACRONYMS AND ABBREVIATIONS $\ldots \ldots \ldots \ldots \ldots \ldots \ldots \ldots \ldots \ldots \ldots \ldots \ldots \ldots \ldots \ldots$

CONVERSION FACTORS $\ldots \ldots \ldots \ldots \ldots \ldots \ldots \ldots \ldots \ldots \ldots \ldots \ldots \ldots \ldots \ldots \ldots \ldots \ldots \ldots$

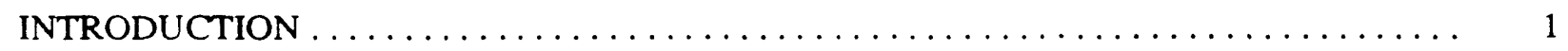

TAB SECTIONS

INEL Record-to-Date Summaries

INEL 1990 Summaries

Argonne National Laboratory - West, 1990 Detail Data

Central Facilities Area, 1990 Detail Data

Chemical Processing Plant, 1990 Detail Data

Containment Test Facility, 1990 Detail Data

Magnetohydrodynamics Component Development, 1990 Detail Data

Naval Reactors Facility, 1990 Detail Data

Power Burst Facility, 1990 Detail Data

Radioactive Waste Management Complex, 1990 Detail Data

Test Reactor Area, 1990 Detail Data

Technical Support Facility - Test Area North, 1990 Detail Data

Water Reactor Research Test Facility - Test Area North, 1990 Detail Data 


\section{ACRONYMS AND ABBREVIATIONSa}

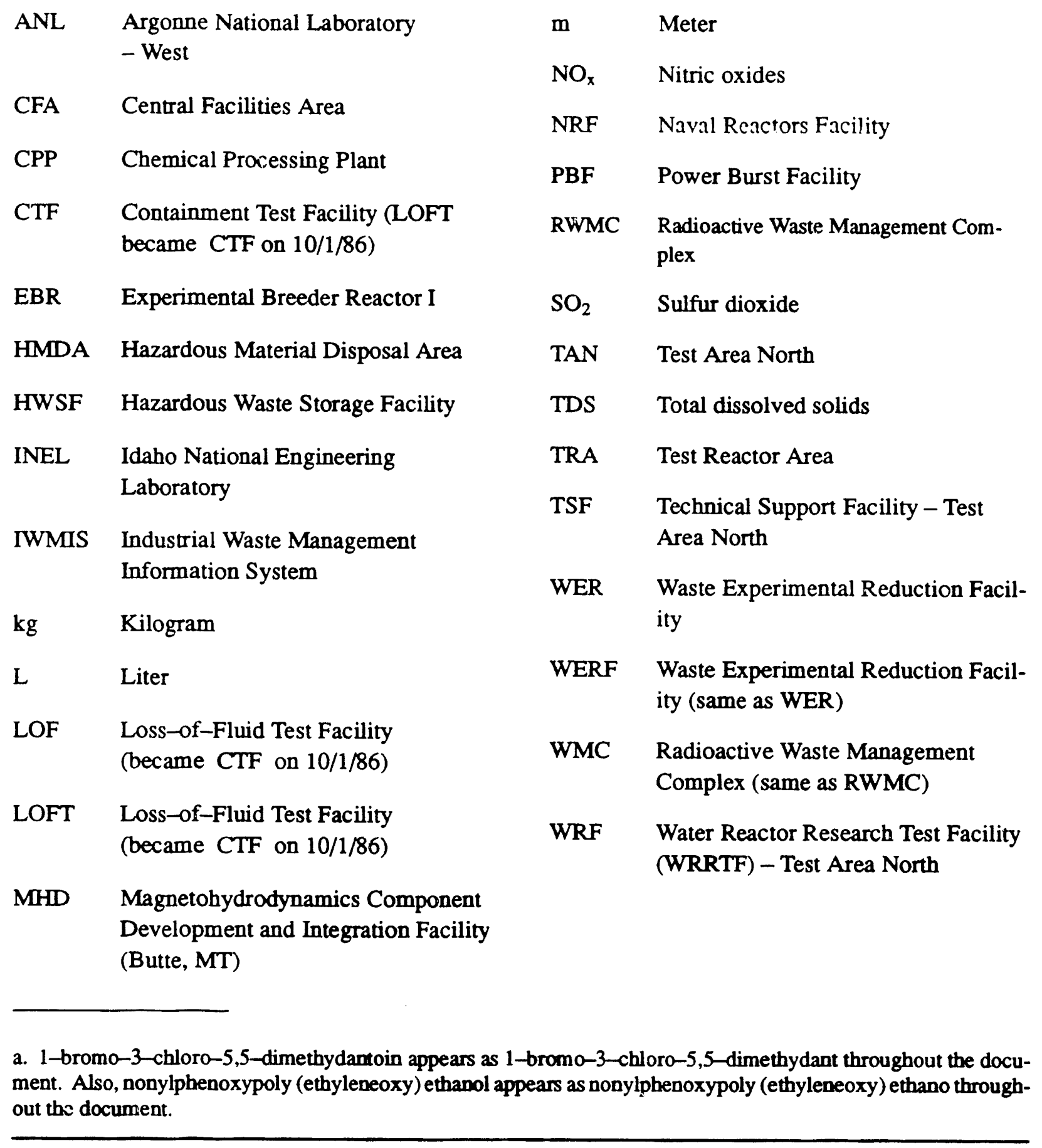




\section{CONVERSION FACTORS}

To Convert From

Cubic meters

Liters

Kilograms
To

Multiply By

Cubic feet

Gallons (U.S. liquid)

Pounds
35.314

0.2642

2.2046 


\section{INDUSTRIAL WASTE MANAGEMENT INFORMATION FOR 1990 AND RECORD-TO-DATE}

\section{INTRODUCTION}

This report summarizes industrial waste data records for the Idaho National Engineering Laboratory (INEL) for 1990 and record-to-date. It includes summaries of the quantities of liquid industrial waste, the volumes of solid waste, the fuel oil and water used, and the quantities of $\mathrm{SO}_{2}$ and particulates released. The waste data are from the INEL Industrial Waste Management Information System (IWMIS), a computerized database that has been used at the INEL since 1971. An INEL Waste Information Task Force determined solid hazardous waste reporting in the IWMIS should discontinue. Figures have been revised to reflect this request.

This report provides graphics to aid in the presentation of the INEL summary data. The graphics display volumes of water and fuel oil used, volumes of solid waste discarded at the landfill, and weights of chemicals released as liquid and airborne effluents for the record-to-date and the current year.

Some summaries in this report address wastes by type. The types of industrial waste handled at the INEL are:

\begin{tabular}{cll} 
Waste Type & & \multicolumn{1}{c}{ Description } \\
\cline { 1 - 1 } 1 & & Trash \\
2 & & Cafeteria Garbage \\
3 & & Wood and Scrap Lumber \\
4 & & Masonry and Concrete \\
5 & & Scrap Metal \\
6 & & Oil \\
7 & & Solvent \\
8 & & Chemicals \\
9 & & Other (must be specified)
\end{tabular}

The INEL Industrial Waste Summary, a chart listing the waste data available from the period 1971 through 1990, is located within the tab section entitled "INEL Record-to-Date Summaries." Also found in this section are tables that summarize INEL record-to date information, and graphics that provide 1980-1990 summaries, and detailed data for solid and liquid industrial waste, sanitary landfill waste, airborne and liquid industrial waste, chemical usage, fuel oil usage, and water usage.

Charts that summarize INEL industrial waste information for the period January through December 1990 are located in the tab section "INEL 1990 Summaries." This section begins with tables that summarize both the solid waste and the airborne and liquid waste generated at each INEL facility. Additional detailed reports and summaries follow that include Reports 2, 3, and 6.

\section{Report 2 - INEL Water Usage Summary - Year-to-Date}

This report provides the quantity of water pumped and discharged and the percent accountability at each facility for the year-to-date.

\section{Report 3 - INEL Industrial Waste Summary}

This report summarizes the December and year-to-date quantity and type of industrial waste for each INEL facility. The waste is shown in Types 1 through 9 which correspond with the classification for sanitary waste. 


\section{INEL RECORD-TO-DATE SUMMARIES}

INEL Industrial Waste Summary, Record-to-Date $\ldots \ldots \ldots \ldots \ldots \ldots \ldots$ INEL-3

INEL Solid and Liquid Industrial Waste Summary, Record-to-Date . . . . . . . INEL-4

CY 1980-1990 INEL Sanitary Landfill Waste, Graph . . . . . . . . . . . INEL-5

Airborne and Liquid Industrial Waste Substances Summary, Record-to-Date . . . INEL-6

CY 1980-1990 INEL Chemical Usage (Released as Airborne Effiuents),

Graph .........................................

CY 1980-1990 INEL Chemical Usage (Released as Liquid Effluents),

Graph .......................................

INEL Fuel Oil Summary, Record-to-Date $\ldots \ldots \ldots \ldots \ldots \ldots \ldots \ldots \ldots$ INEL-12

CY $1980-1990$ INEL Fuel Oil Usage, Graph . . . . . . . . . . . . . . . . INEL-13

INEL Water Usage Summary, Record-to-Date $\ldots \ldots \ldots \ldots \ldots \ldots \ldots \ldots$ INEL-14

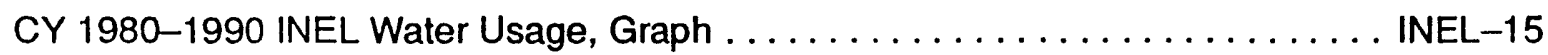

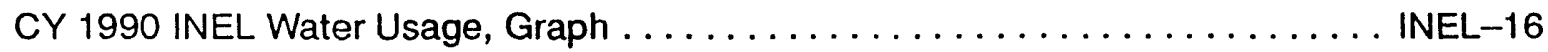




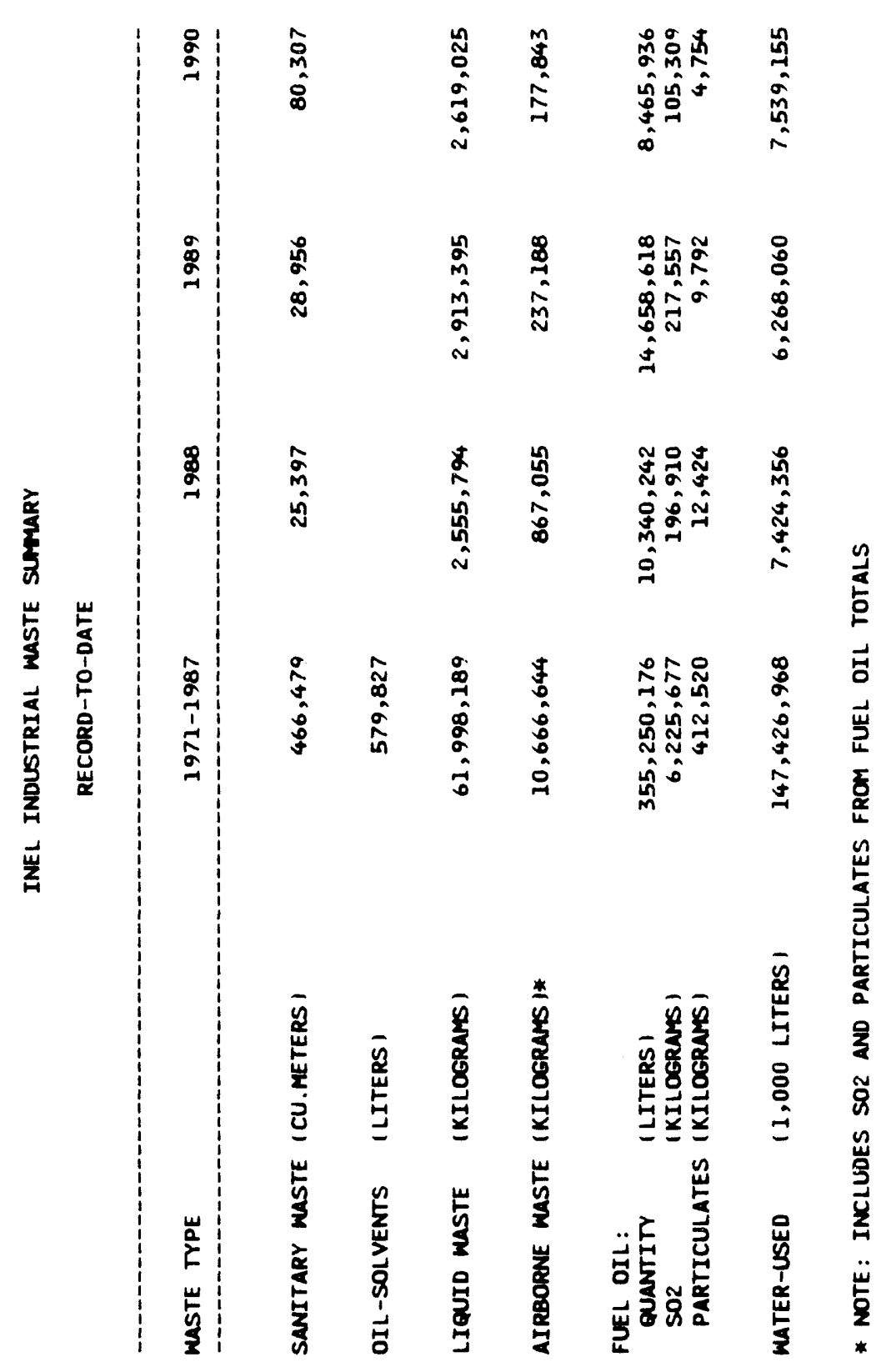

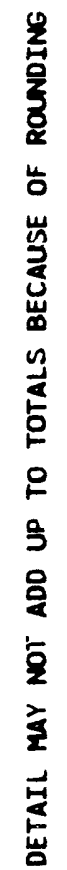

INEL-3 


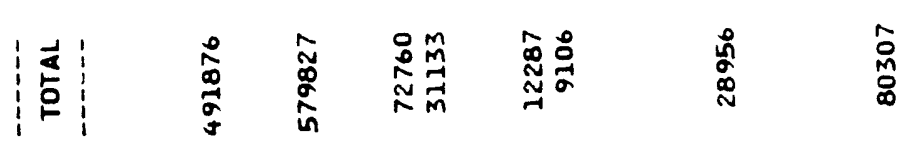

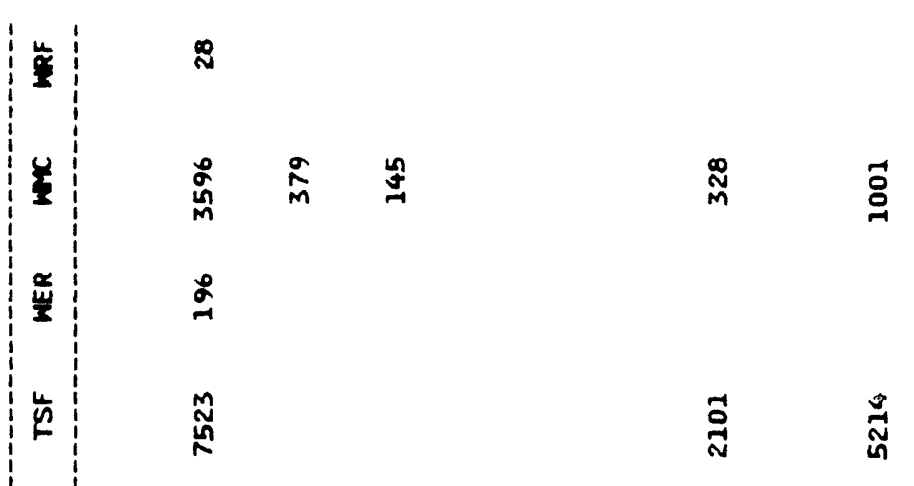

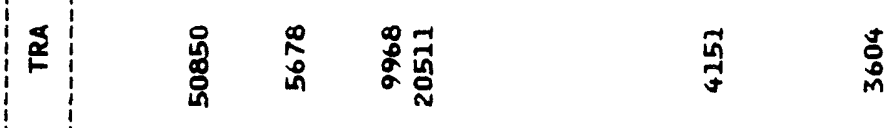

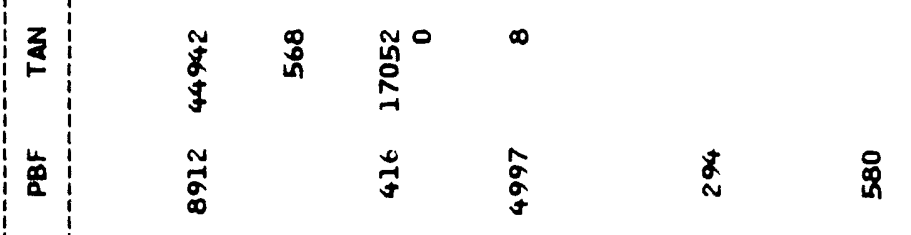

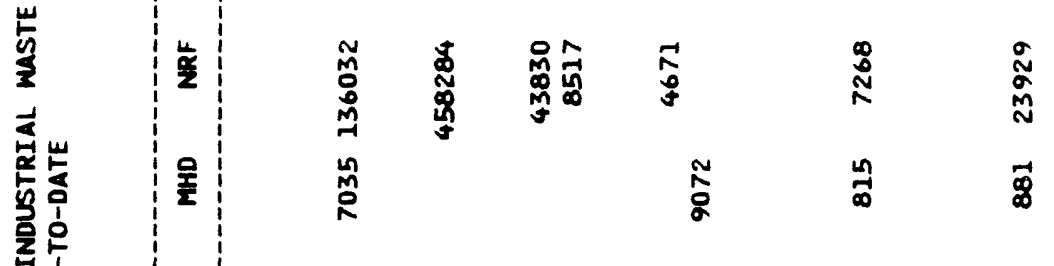

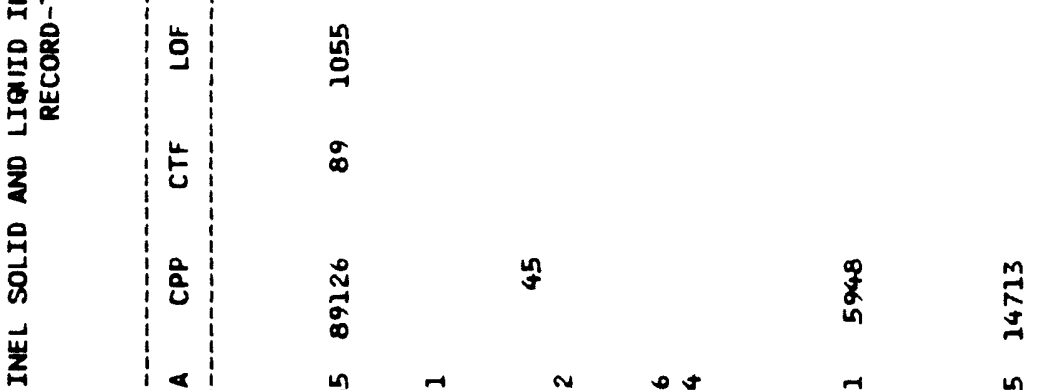

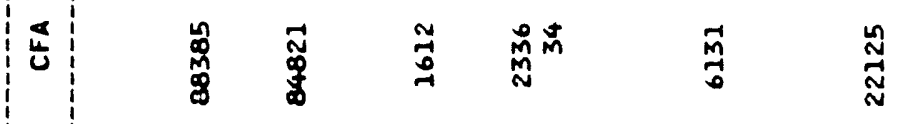

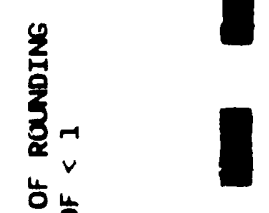

\$

专

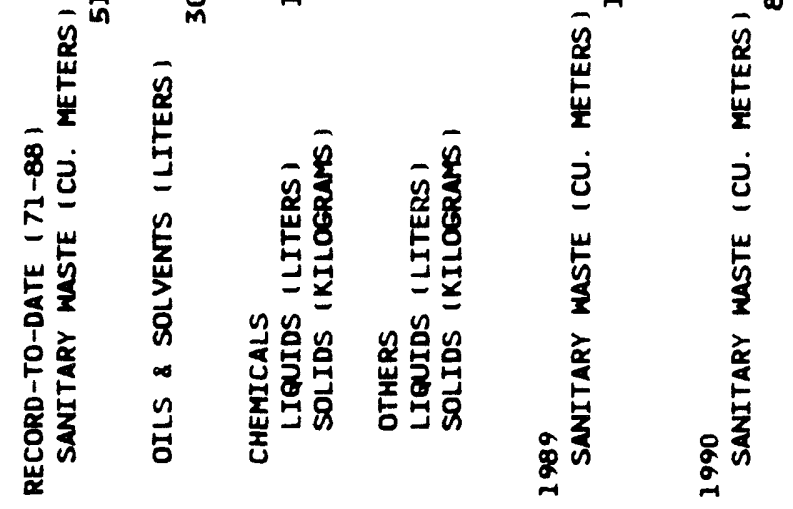

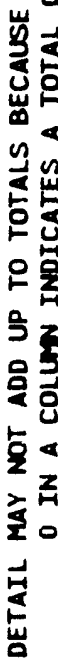

INEL -4 


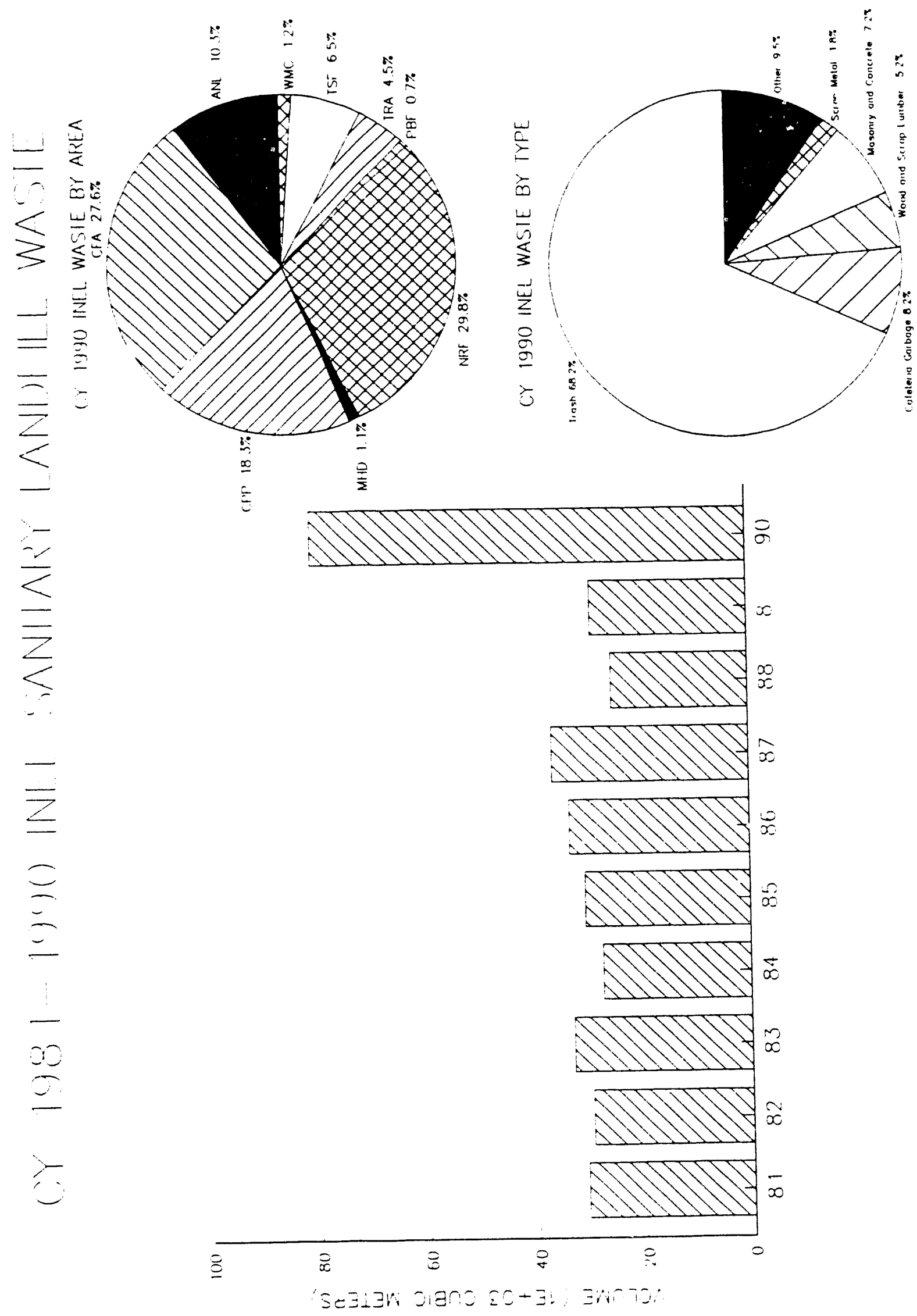

INEL-5 


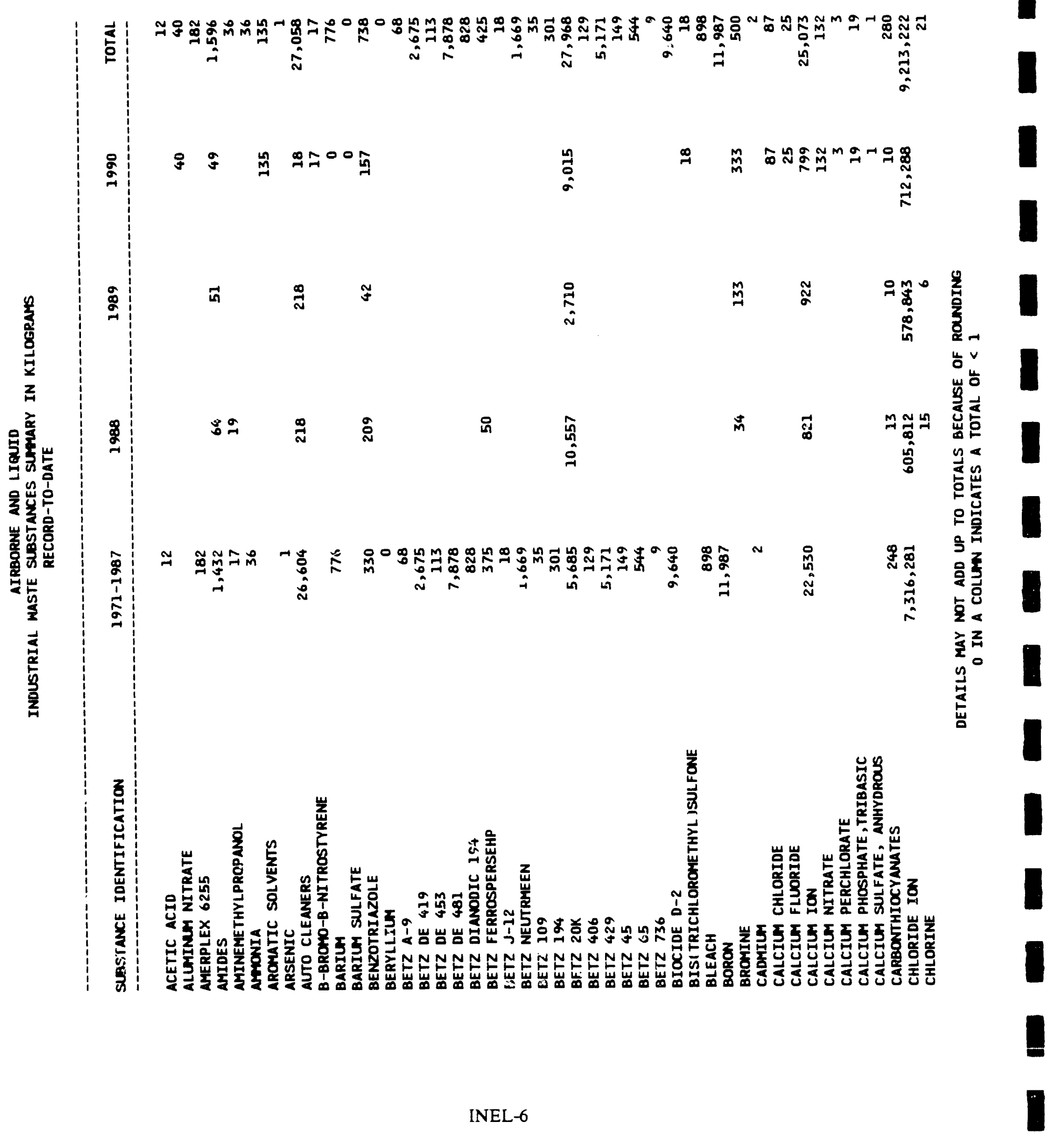




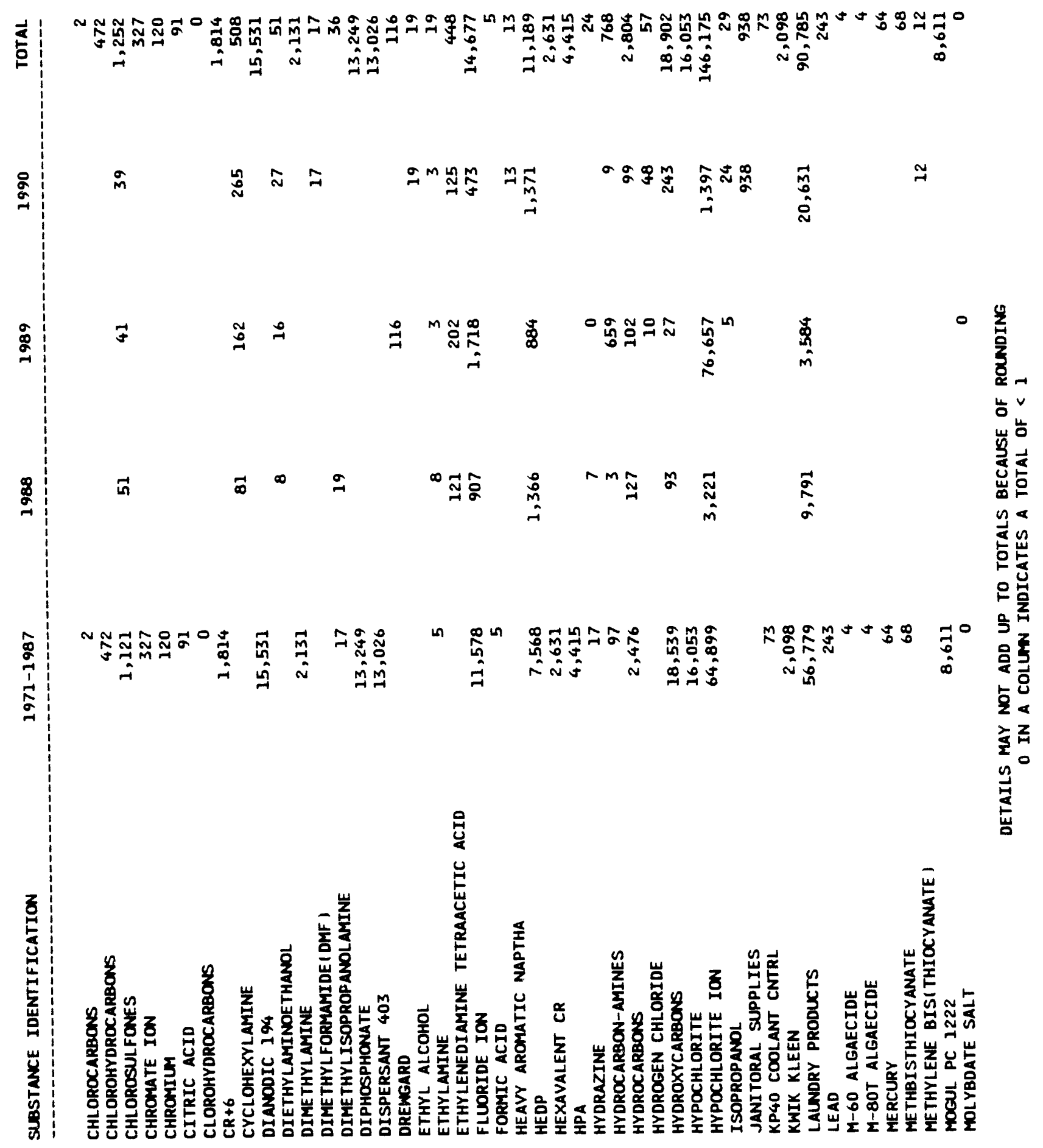




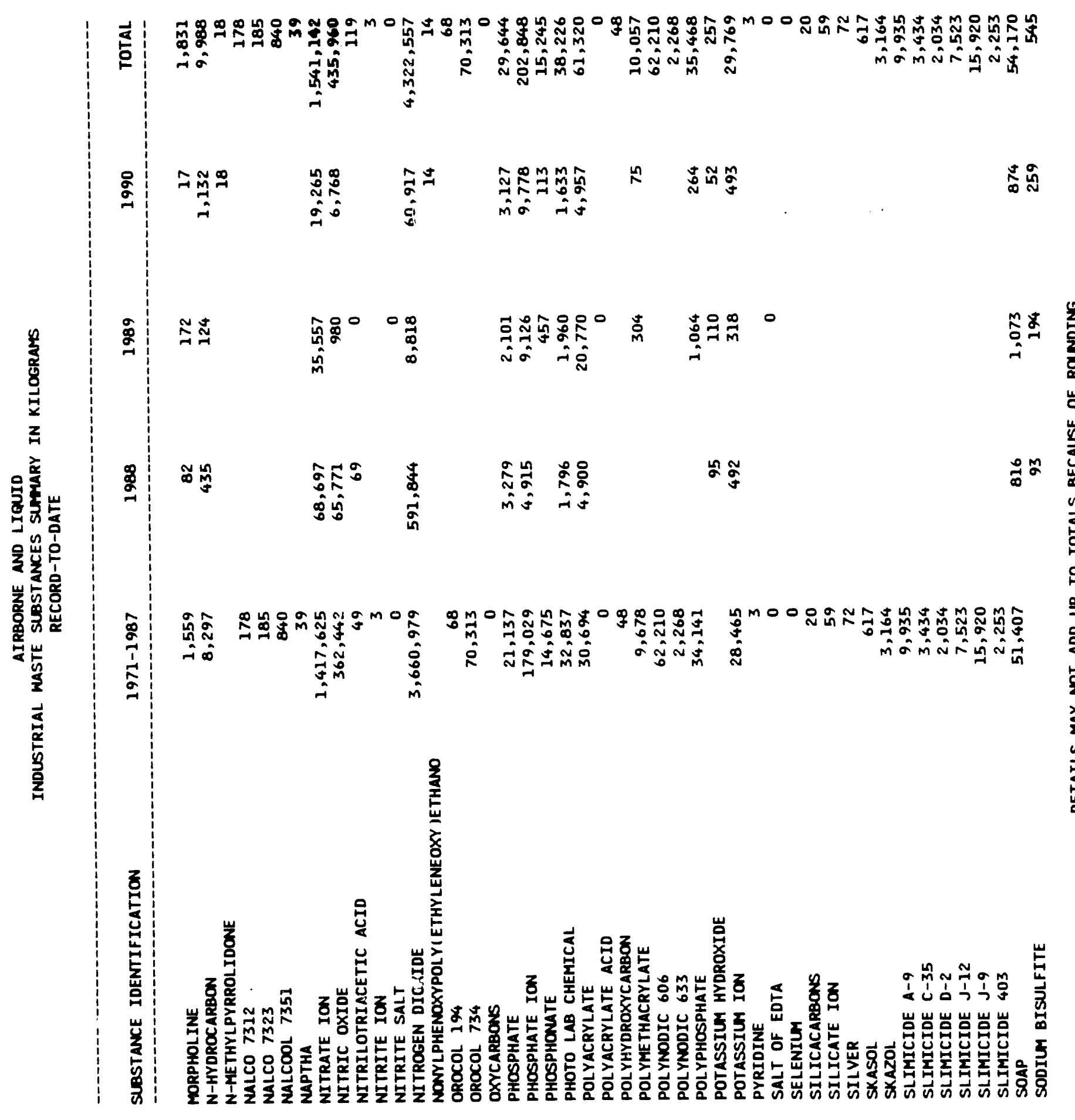




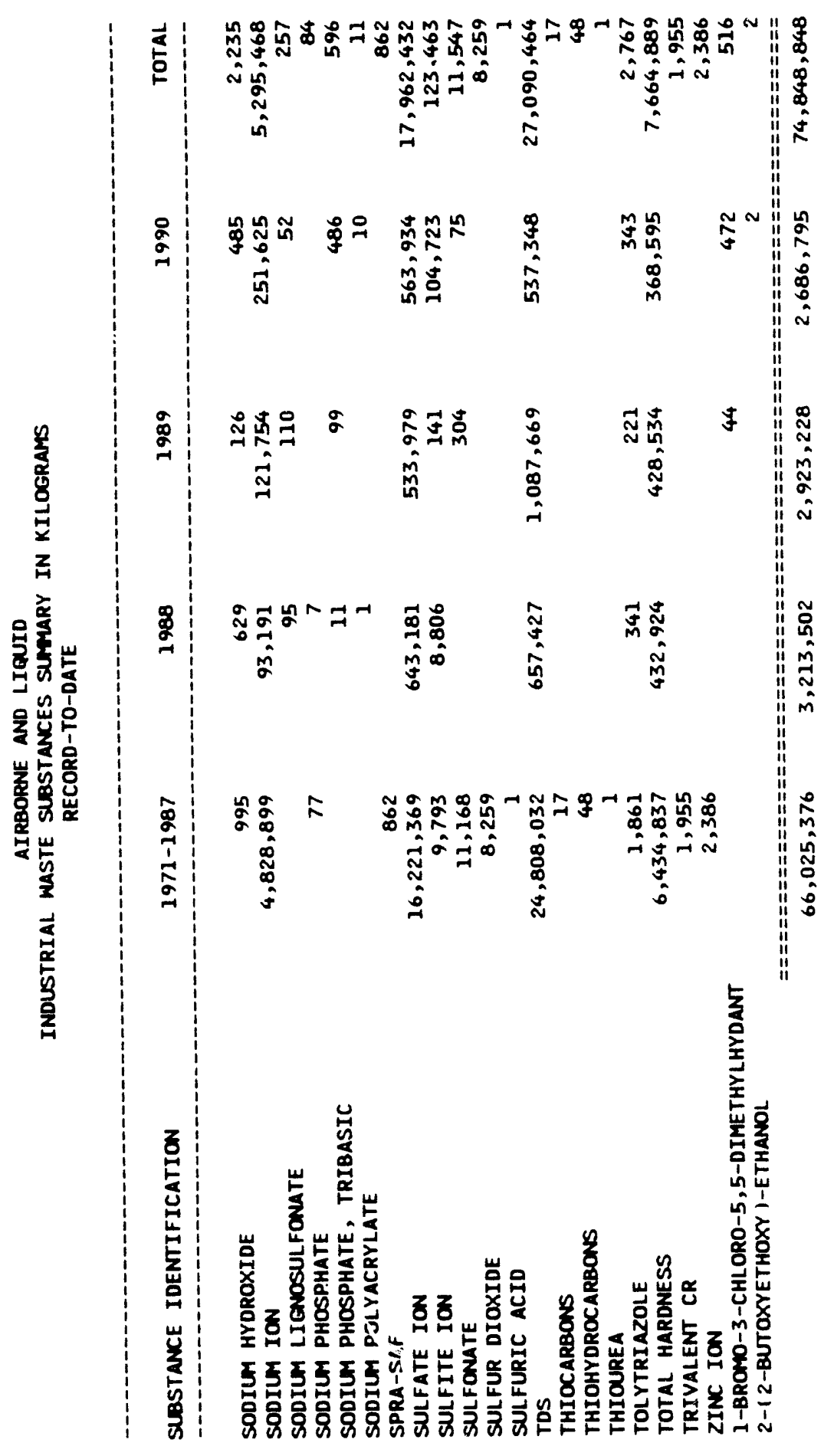




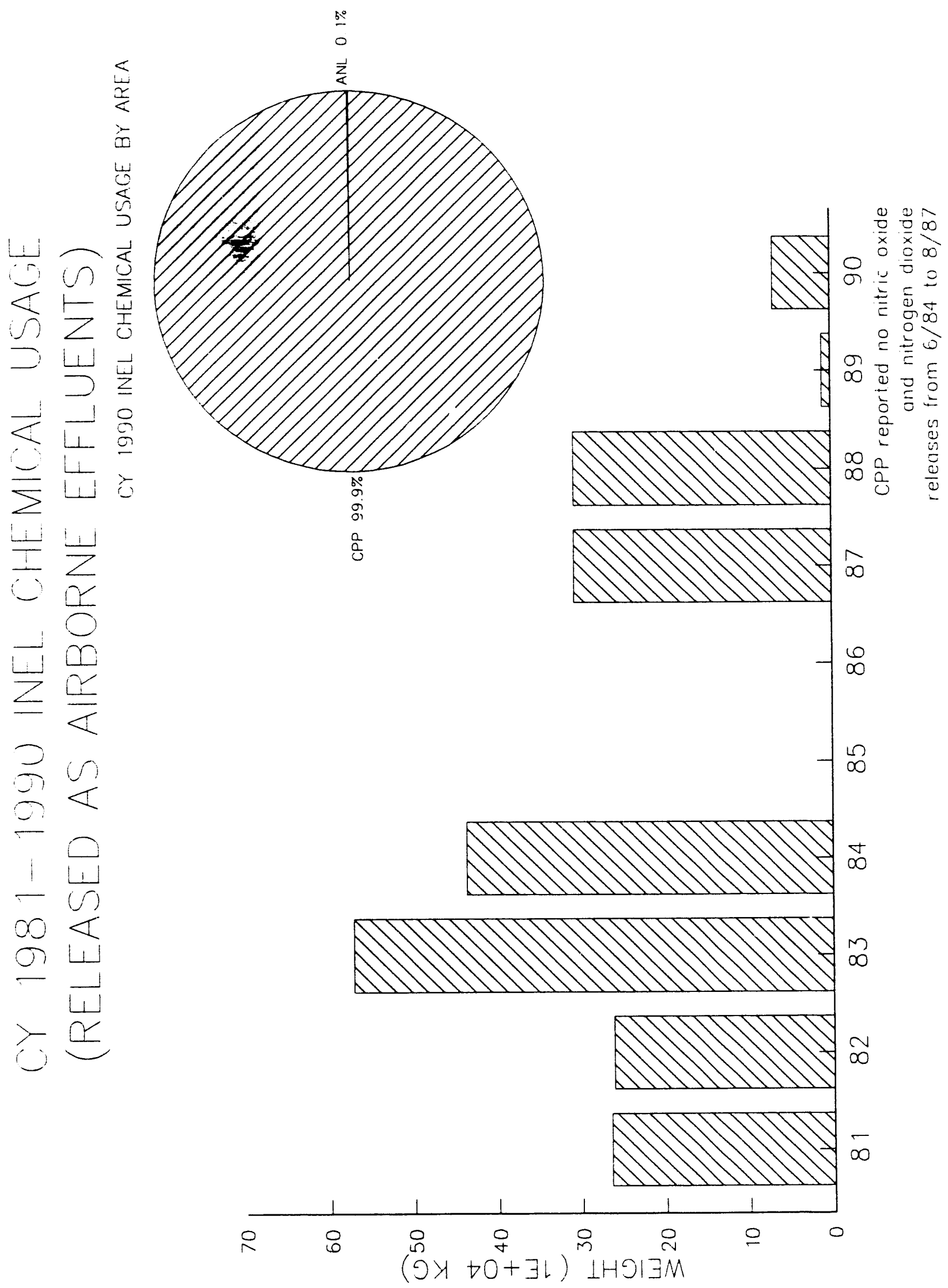




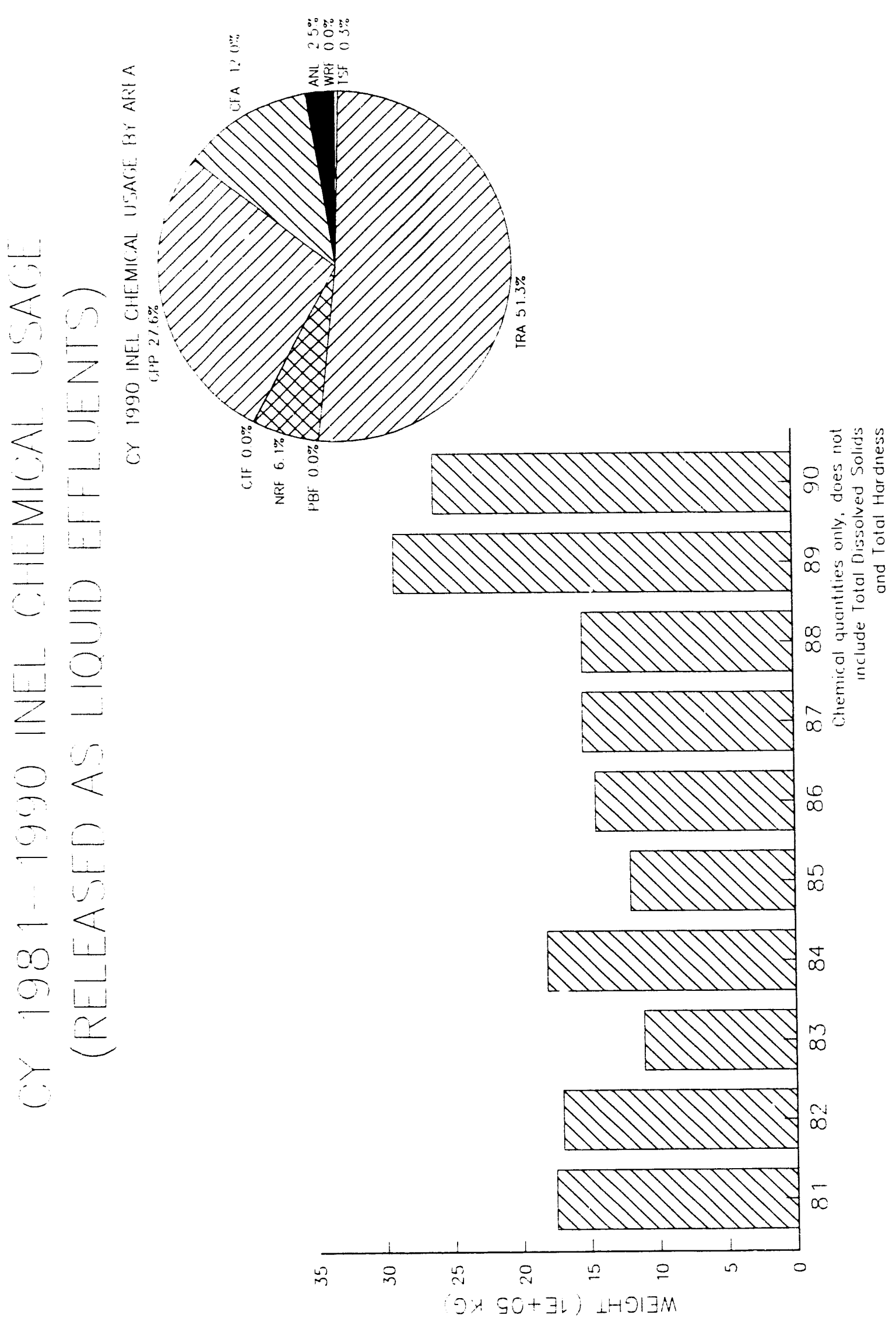



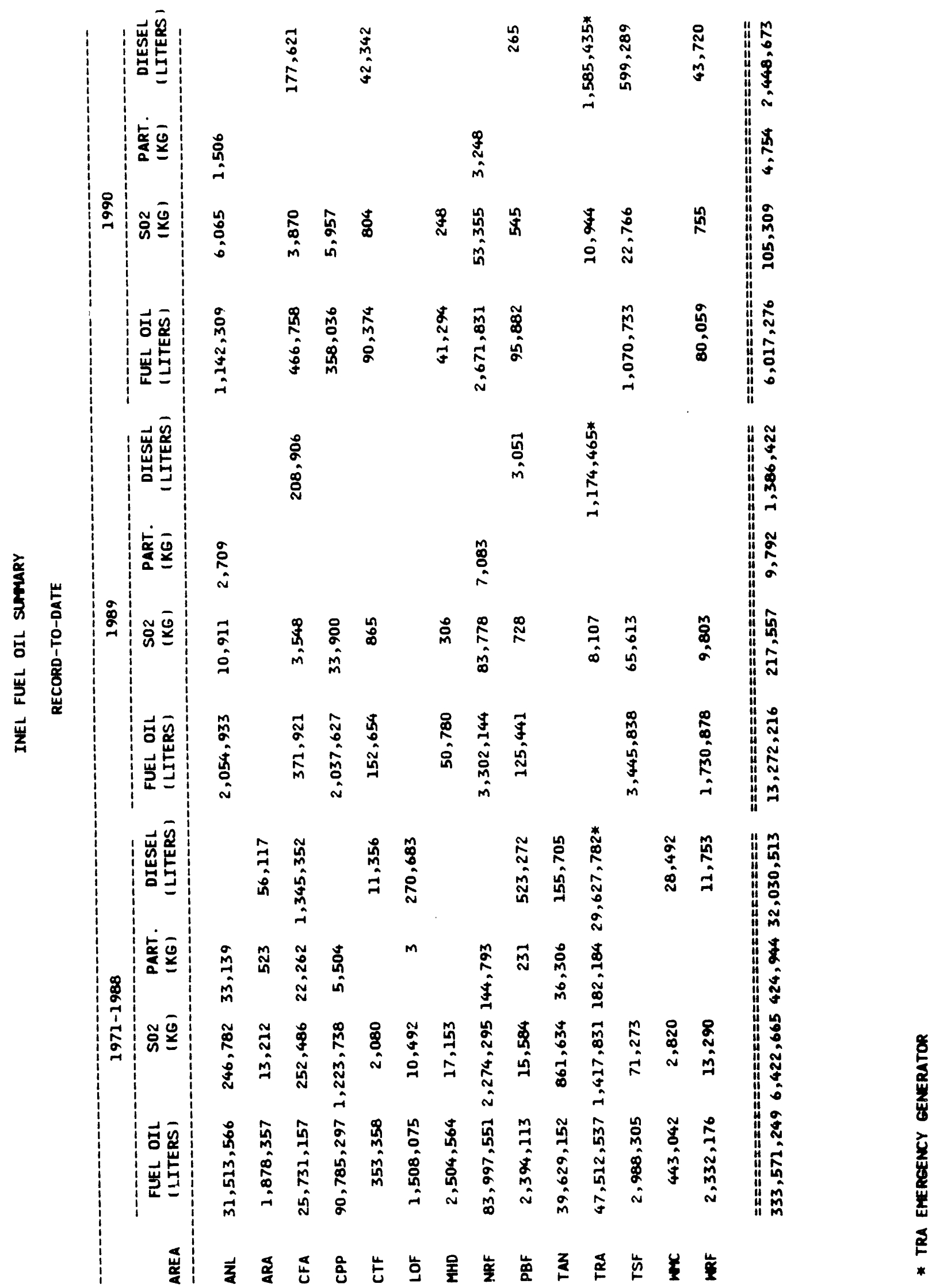

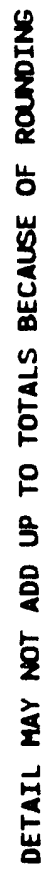




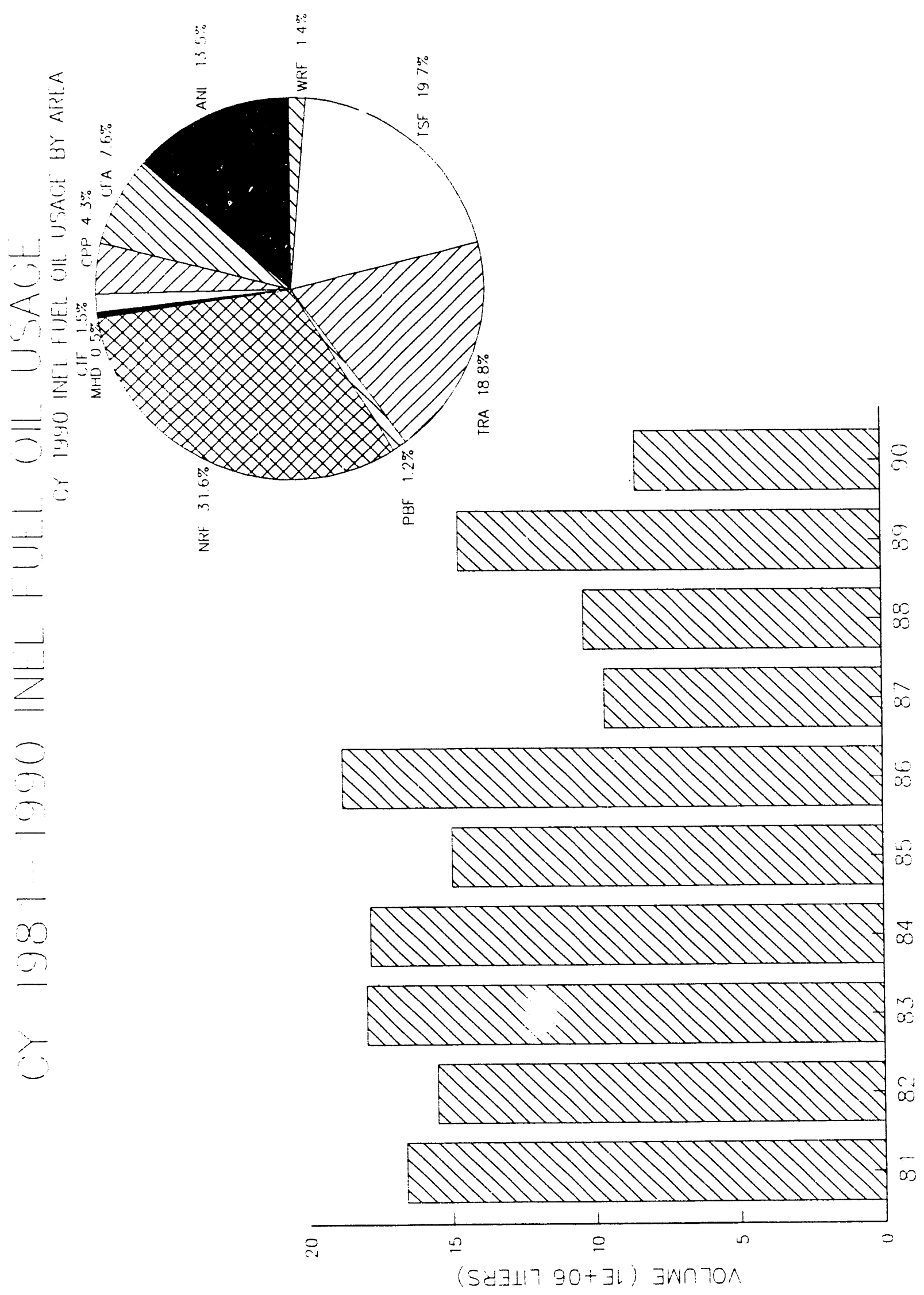

INEL-13 

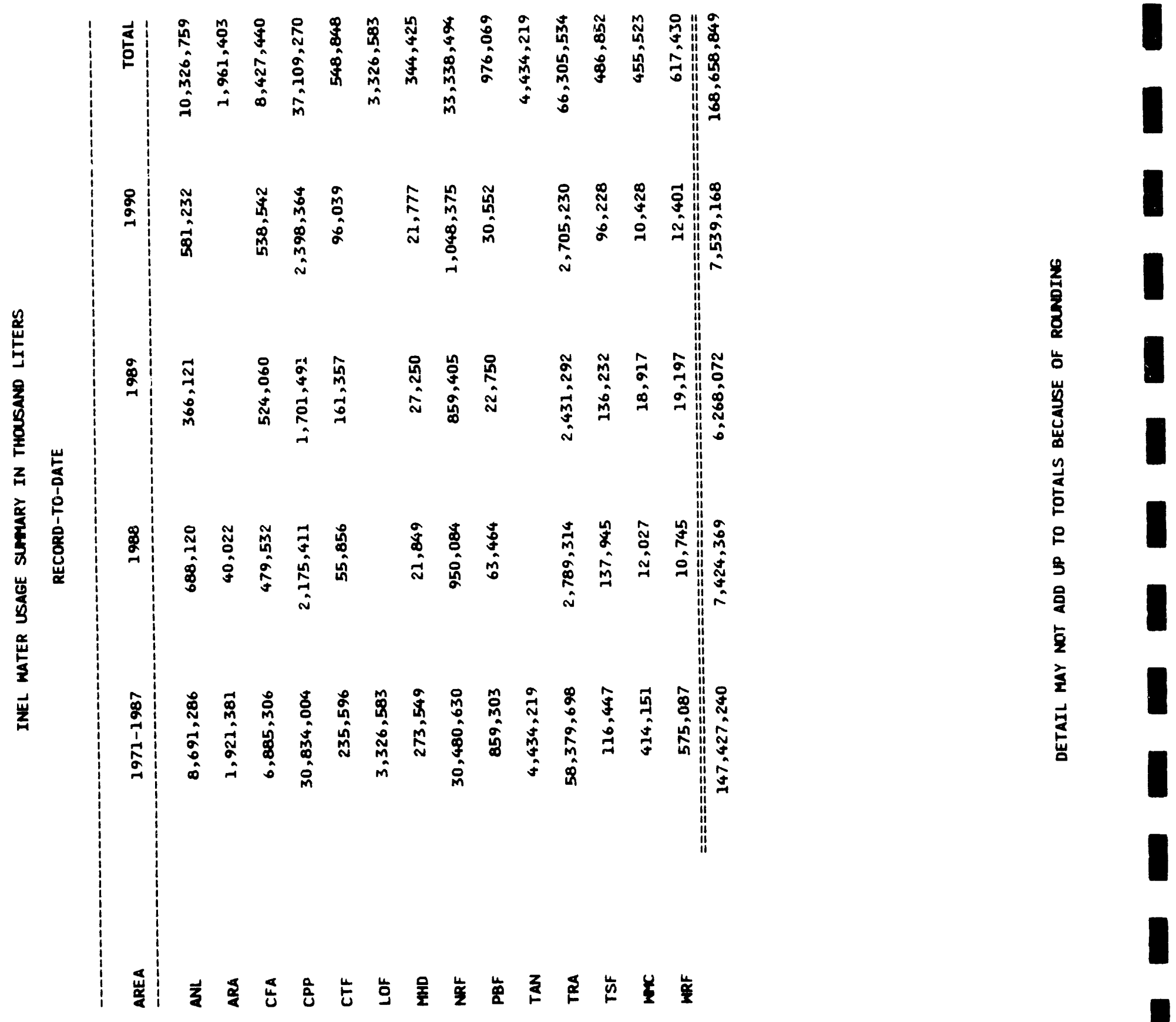


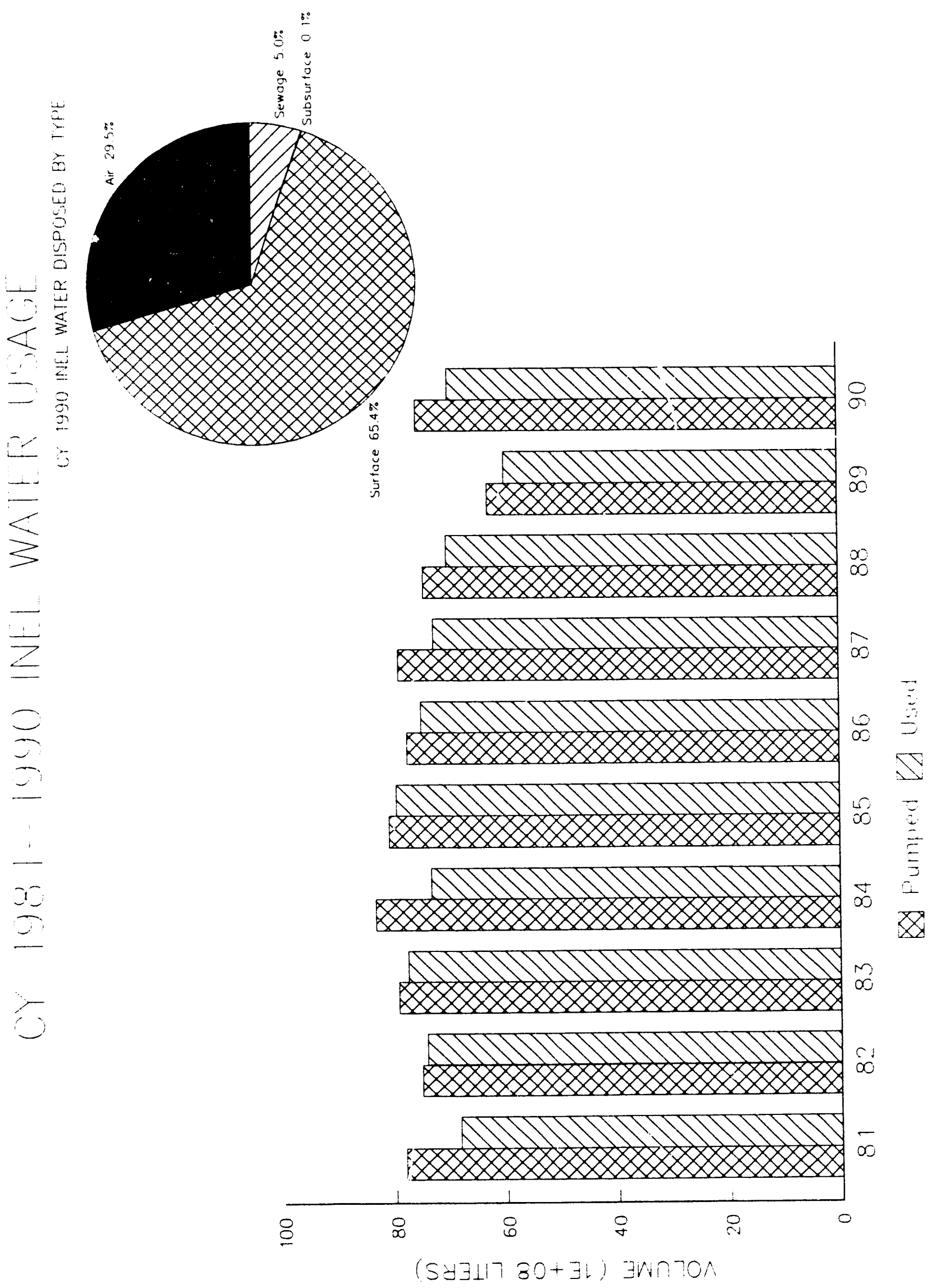

INEL -15 

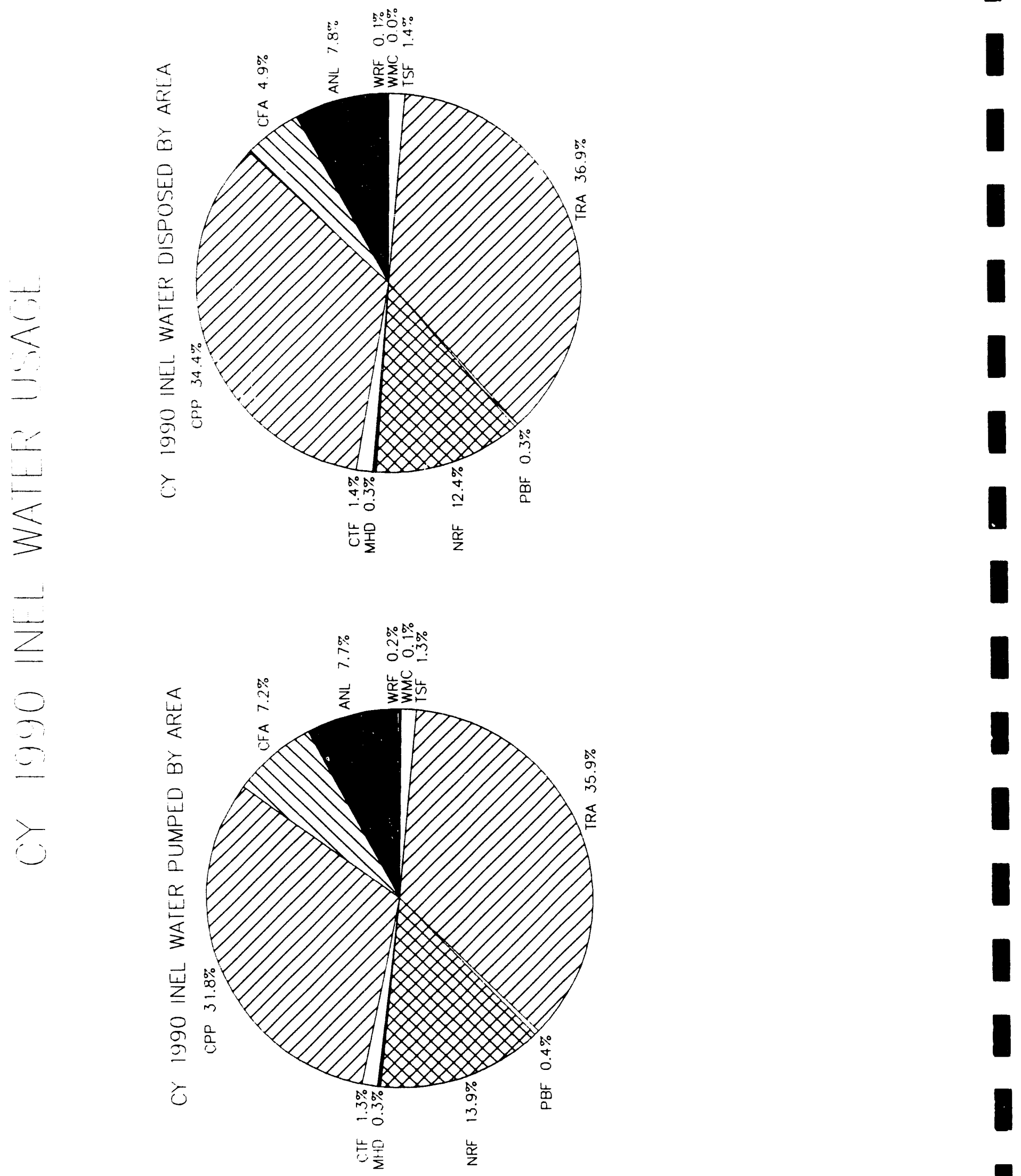


\section{INEL 1990 SUMMARIES}

INEL Industrial Waste Summary, 1990, by Type $\ldots \ldots \ldots \ldots \ldots \ldots \ldots \ldots$ INEL-19

Report 3 - INEL Industrial Waste Summary, December and Year-to-Date by

Facility and Type .................................

Airborne and Liquid Industrial Waste Substances Summary, $1990 \ldots \ldots \ldots \ldots$ INEL-23

Report 2 - INEL Year-to-Date Water Usage Summary . . . . . . . . . . . INEL-25

Report 6 - INEL Year-to-Date Fuel Oil Usage Summary ............. INEL-26 


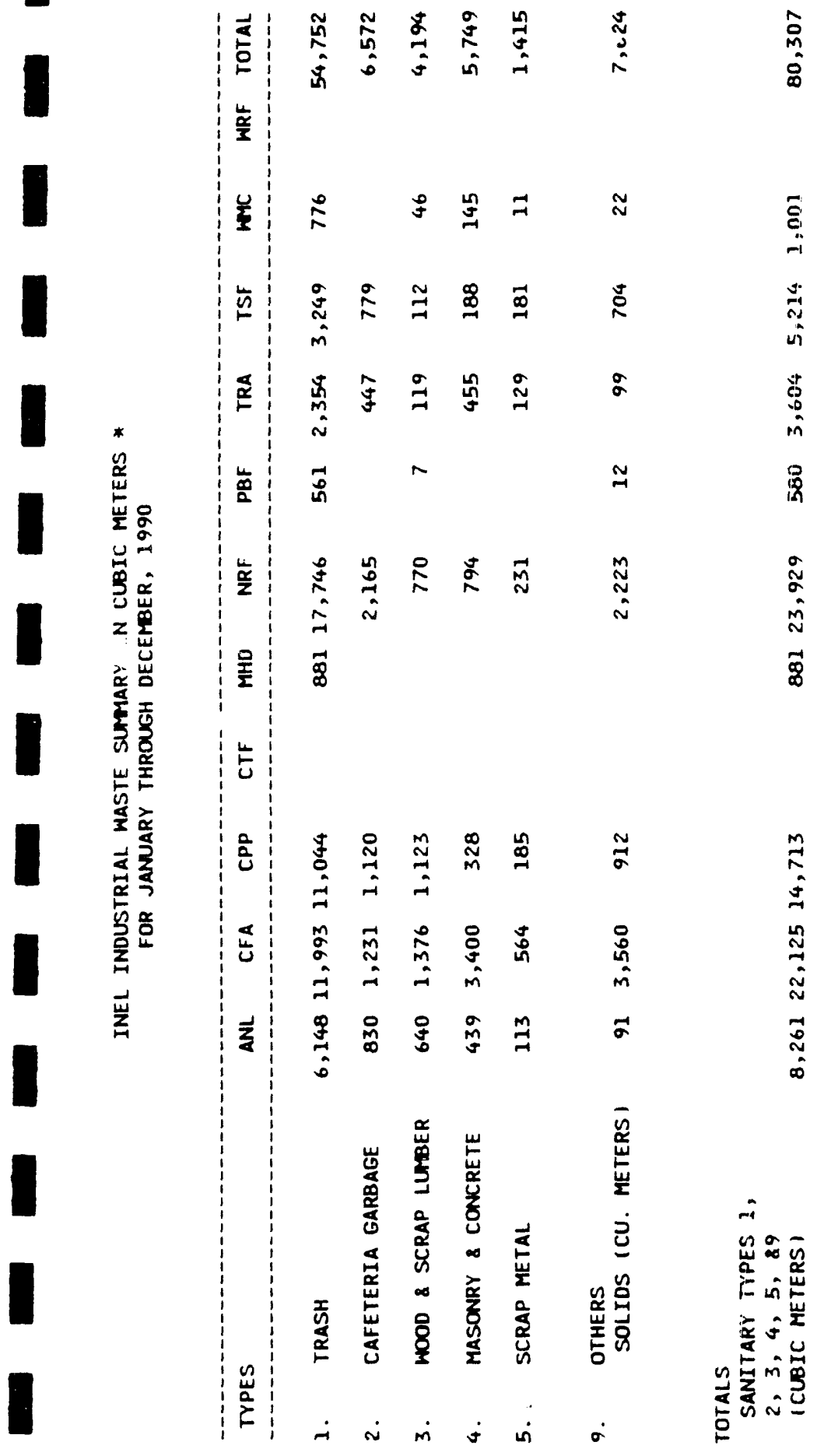




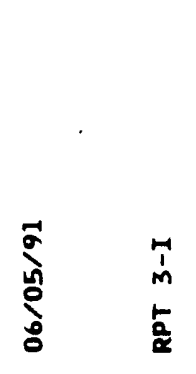

\begin{tabular}{|c|c|c|c|c|}
\hline 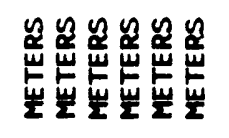 & 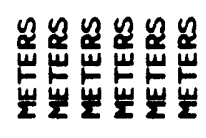 & 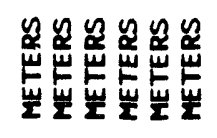 & 旡 & 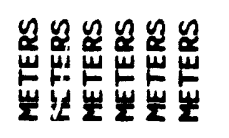 \\
\hline 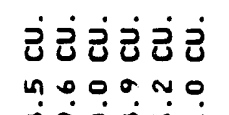 & 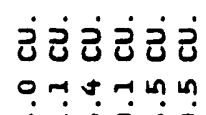 & 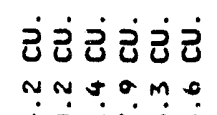 & $\dot{z}$ & 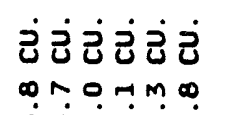 \\
\hline 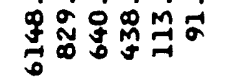 & 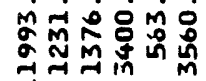 & 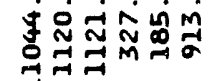 & 富 & 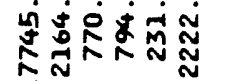 \\
\hline
\end{tabular}
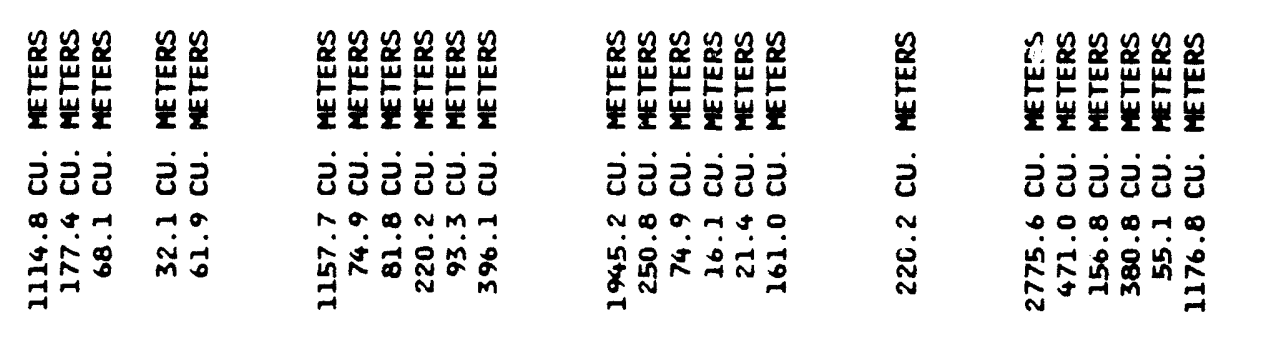

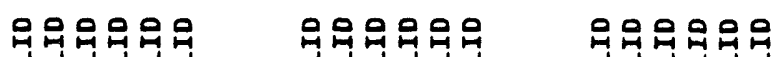

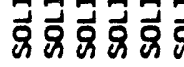
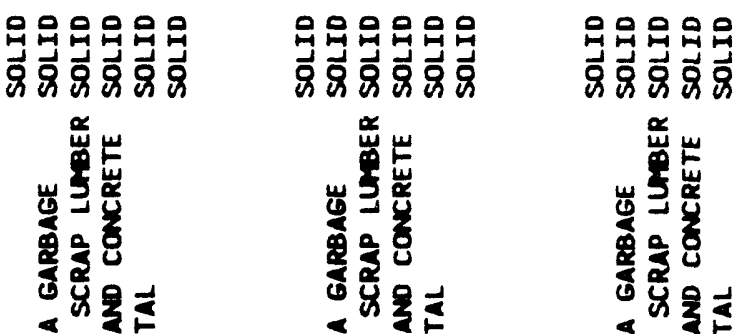

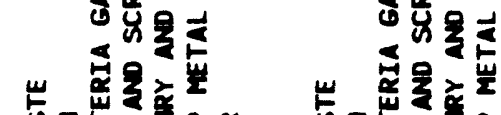

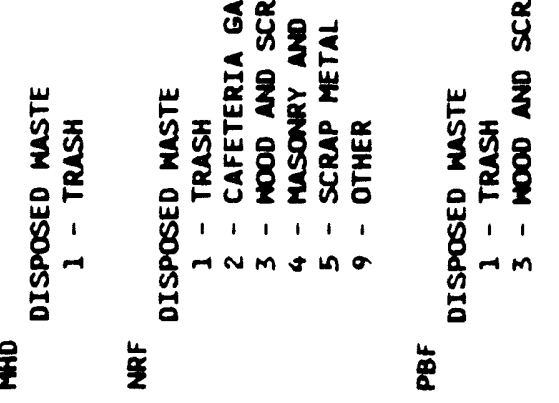



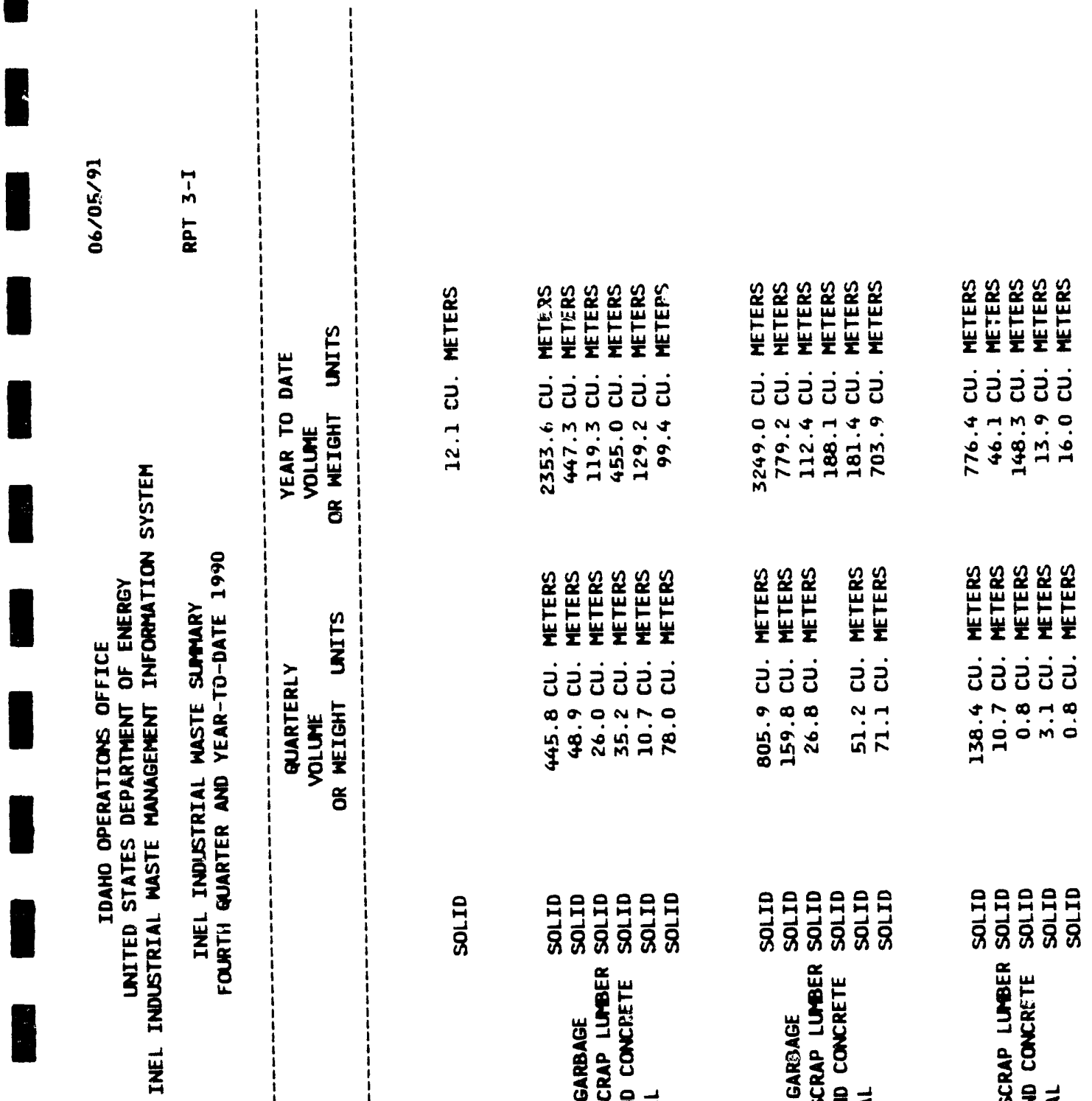

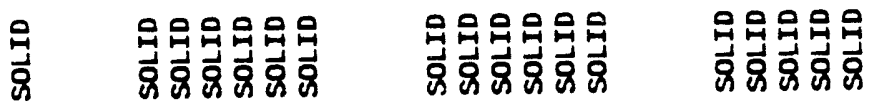
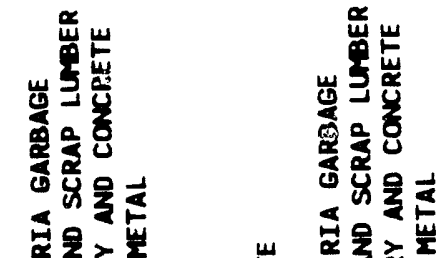

豈崖

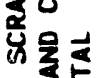

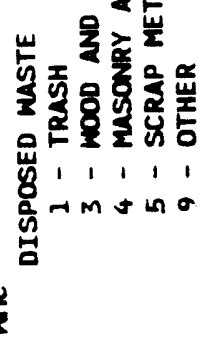

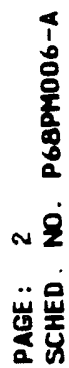

岁1 1111,

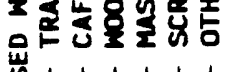

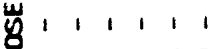

煦

落

革 


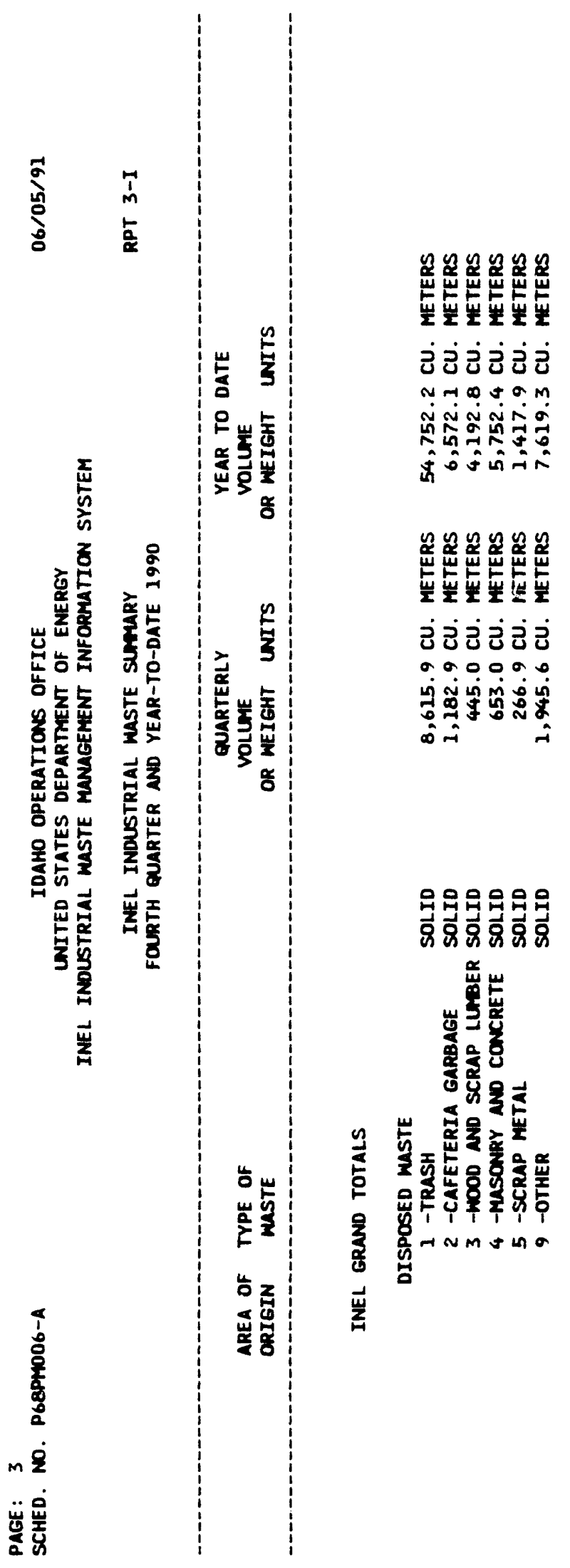




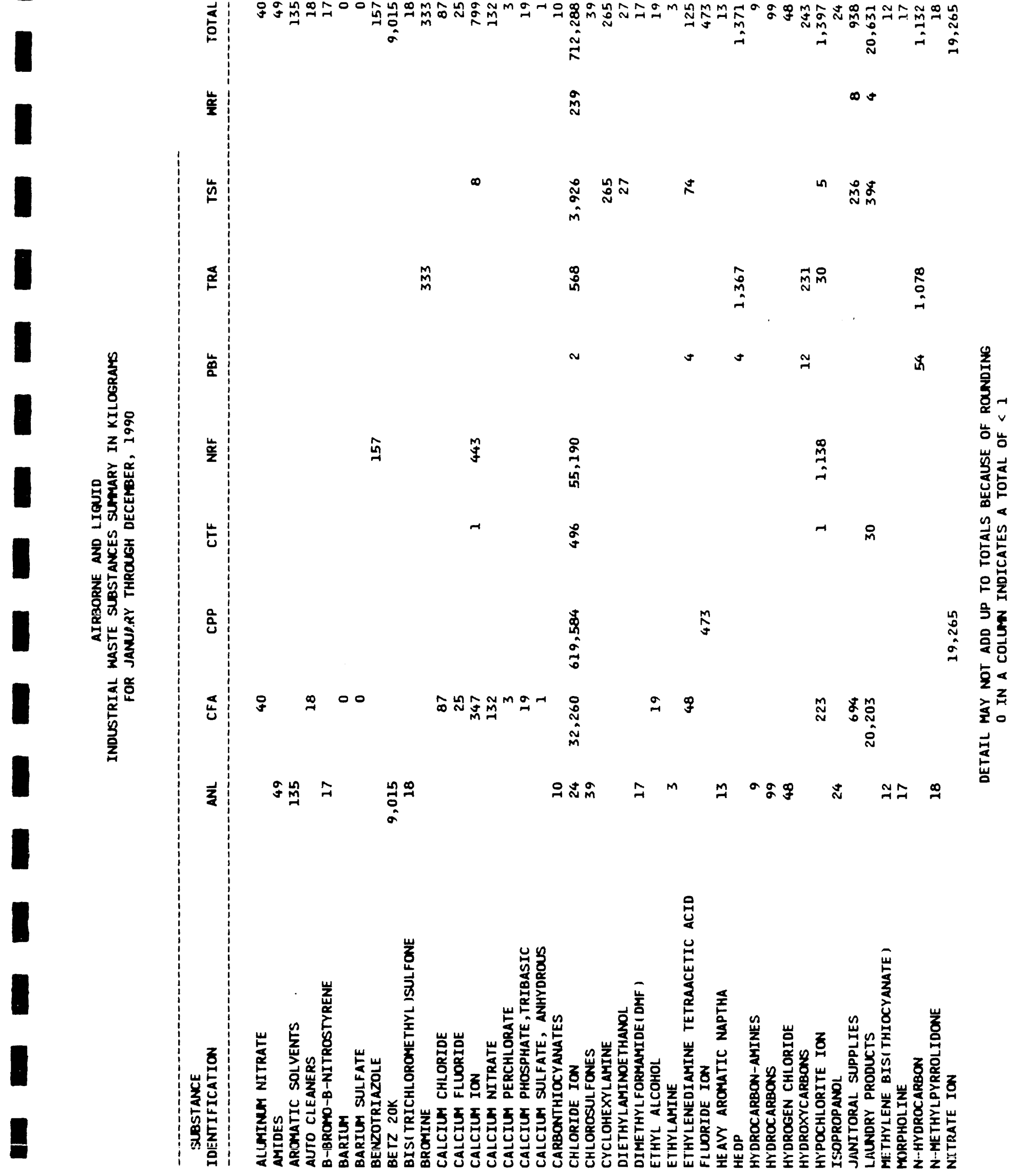

INEL-23 

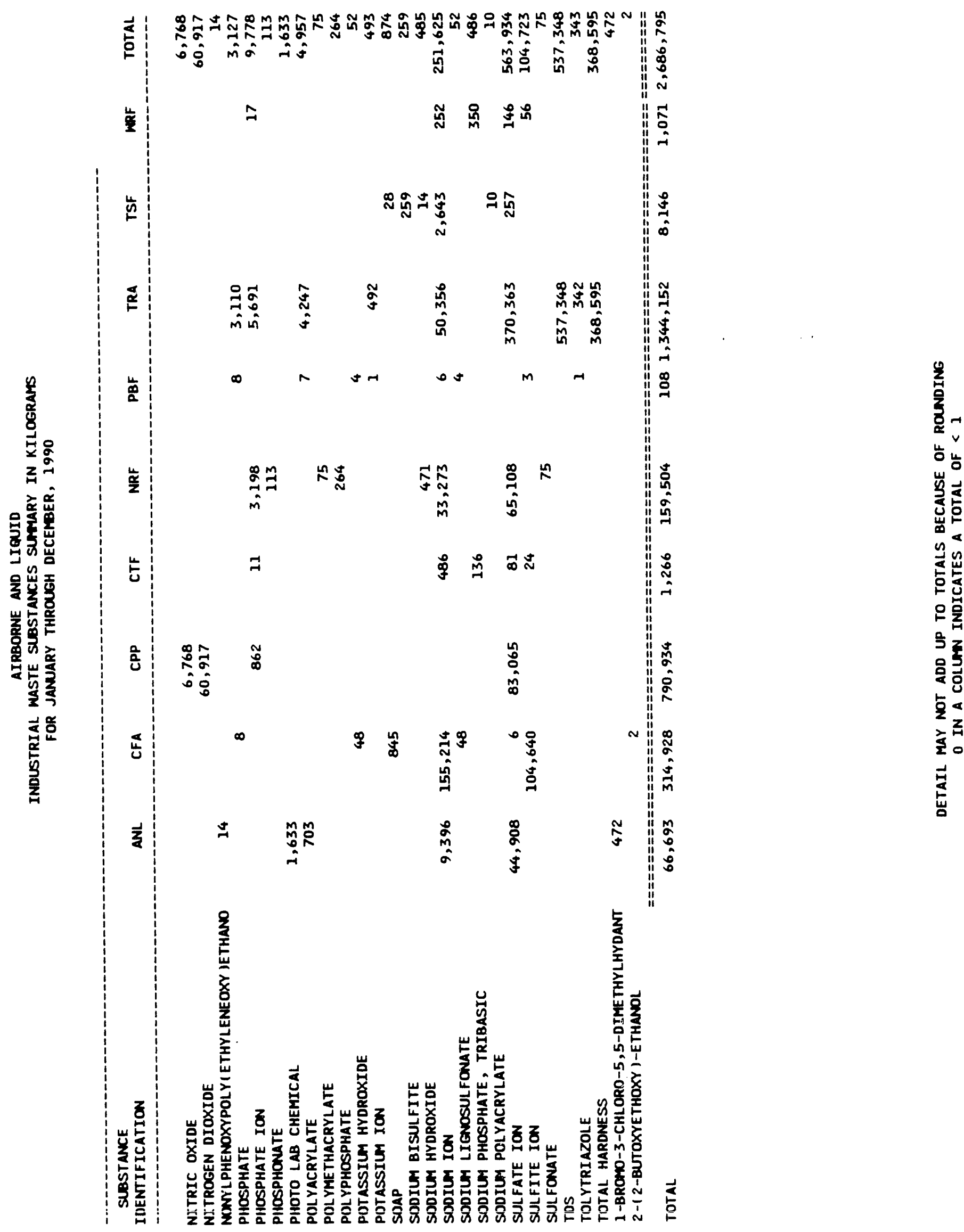

INEL-24 


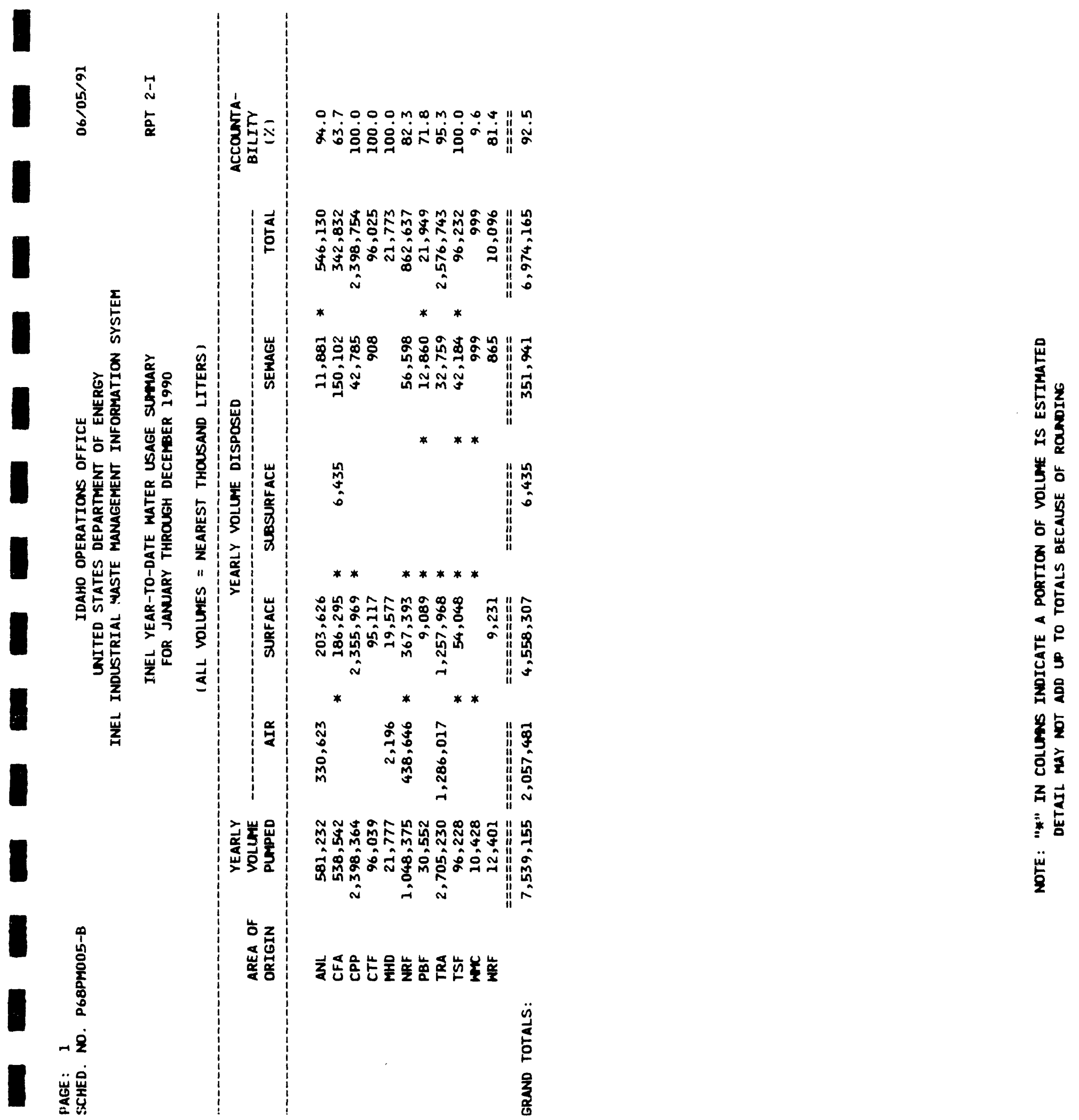




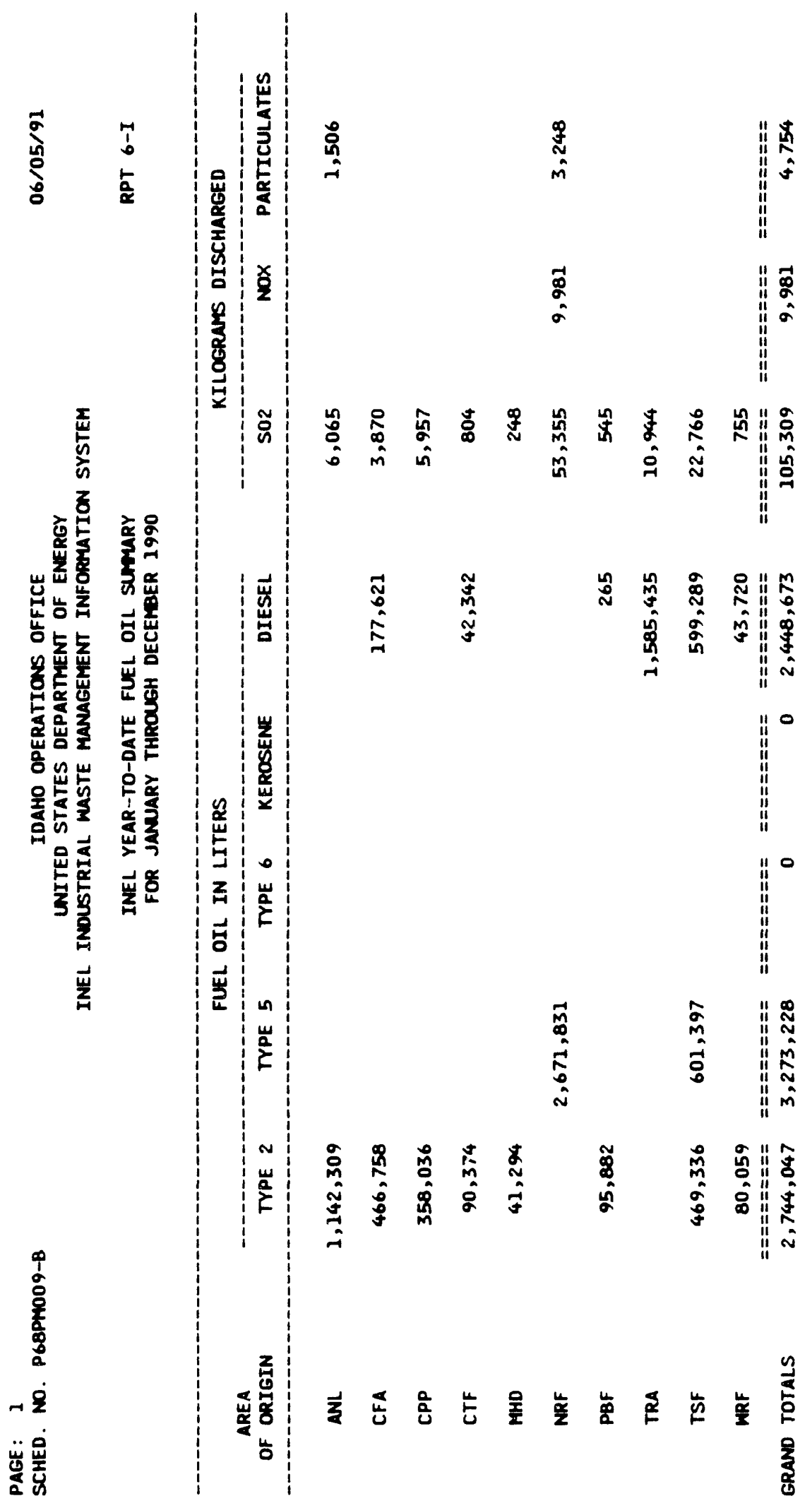

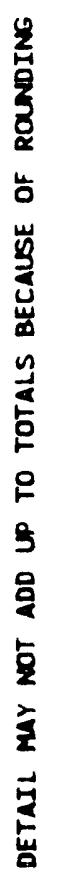

INEL-26 


\section{ARGONNE NATIONAL LABORATORY - WEST, 1990 DETAIL DATA}

Report 114 - ANL Airborne Disposed Substances Summary $\ldots \ldots \ldots \ldots \ldots$ ANL-3

Report 115 - ANL Liquid Disposed Substances Summary $\ldots \ldots \ldots \ldots \ldots$ ANL-5

Report 116 - ANL Industrial Waste Summary $\ldots \ldots \ldots \ldots \ldots \ldots \ldots \ldots \ldots \ldots \ldots$

Report 117 - ANL Water Usage and Disposal Summary .............. ANL-11

Report 118 - ANL Fuel Oil Usage and Stack Effluents Summary .......... ANL-13 
I

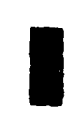

I

I

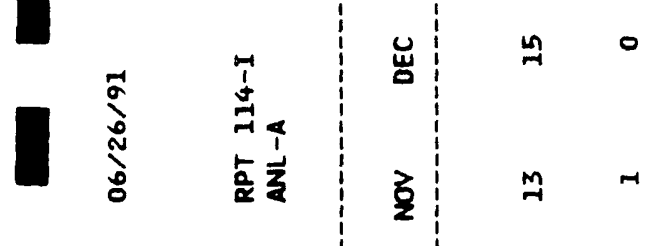

I

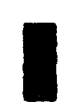

I

$\frac{5}{\frac{5}{5}}$

1

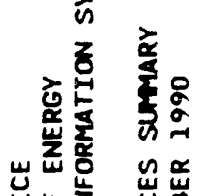

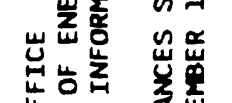

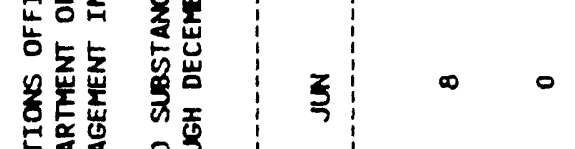

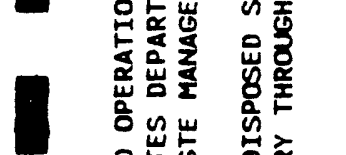

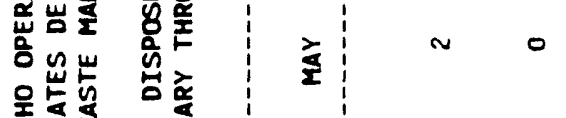

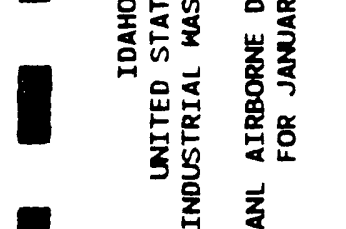

I

专焉

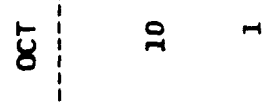

岁

30

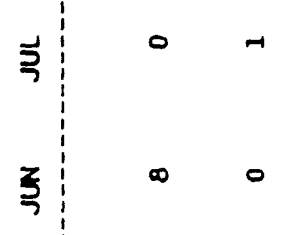

高

\begin{tabular}{l|ll}
$\frac{\alpha}{2}$ & $\infty$ & -1
\end{tabular}

\begin{tabular}{l|ll}
$\mathbb{8}$ & $\infty$ & $\infty$
\end{tabular}

3)

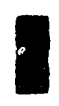

I

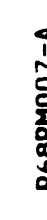

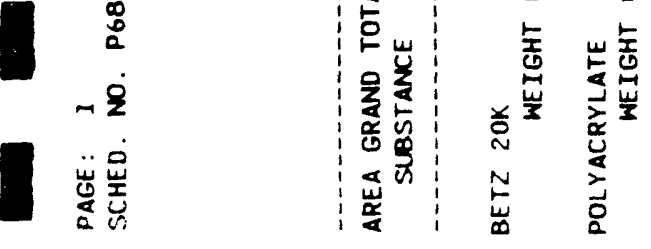




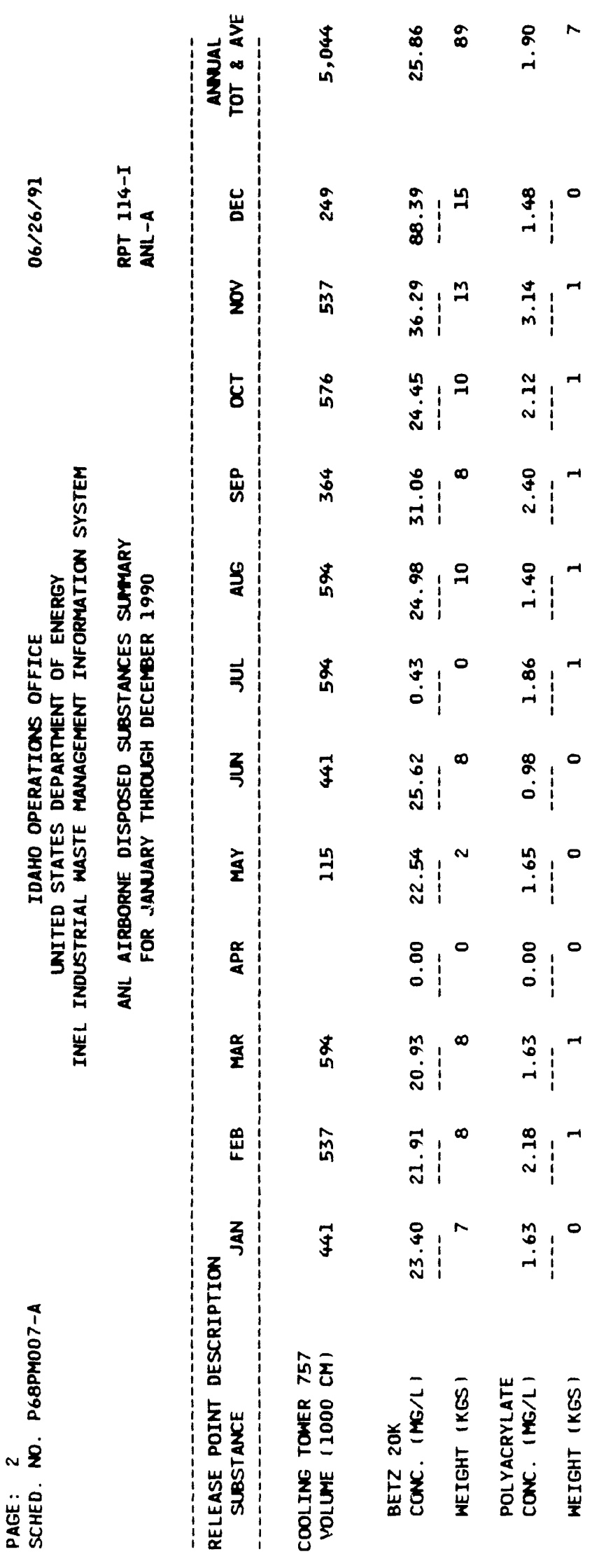




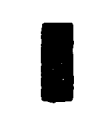

I

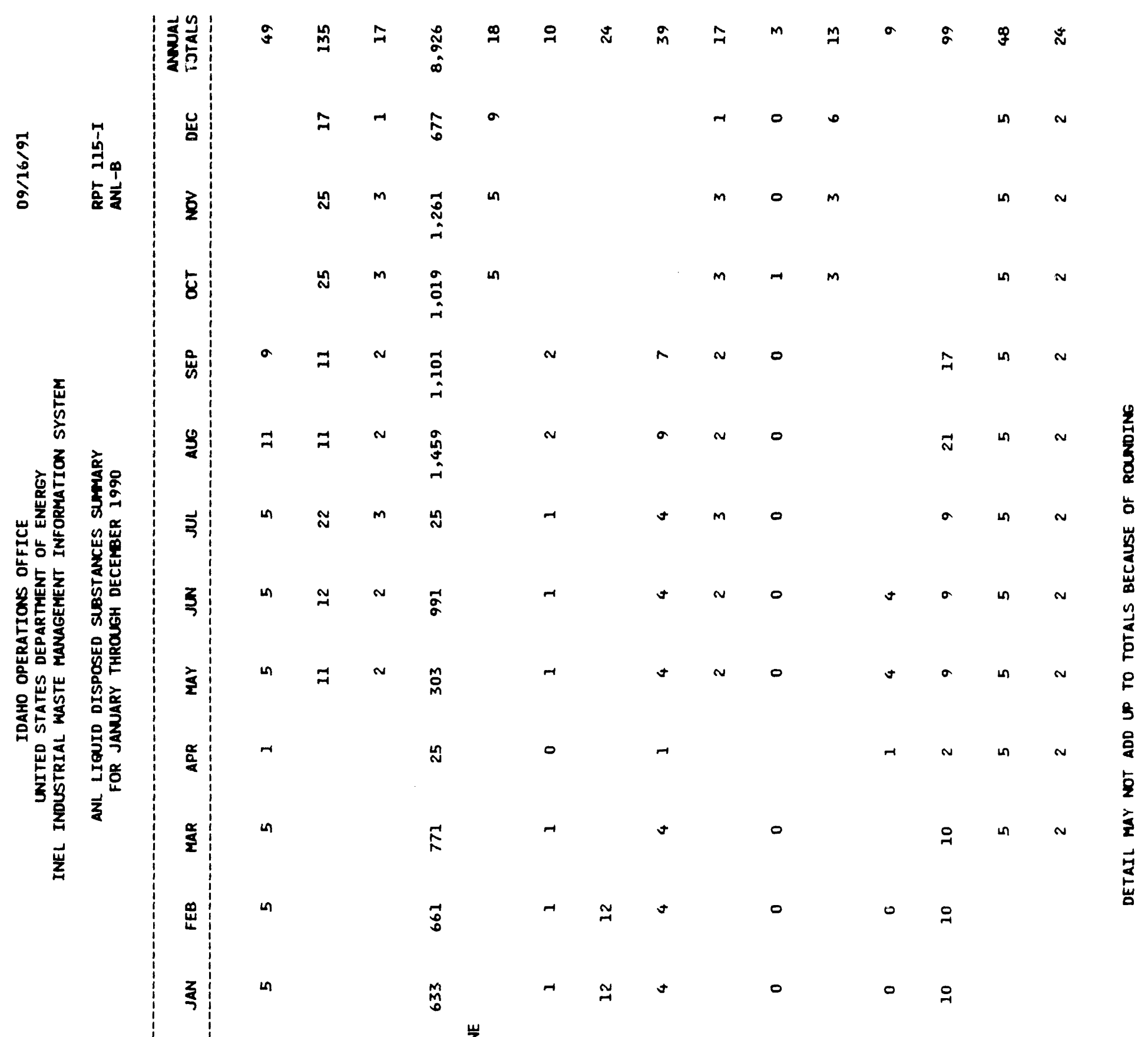

】竞

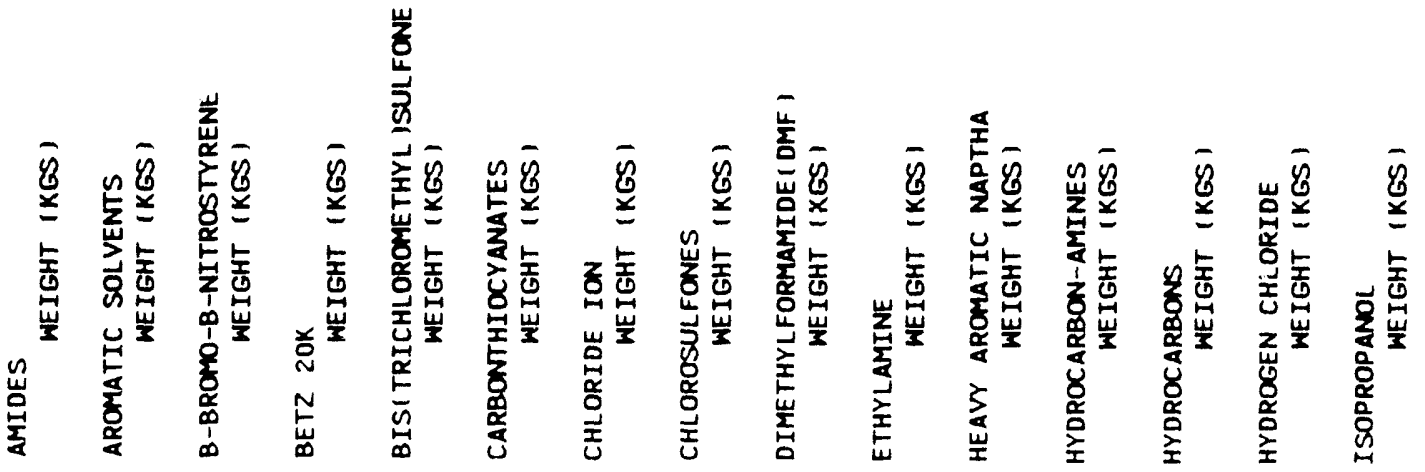




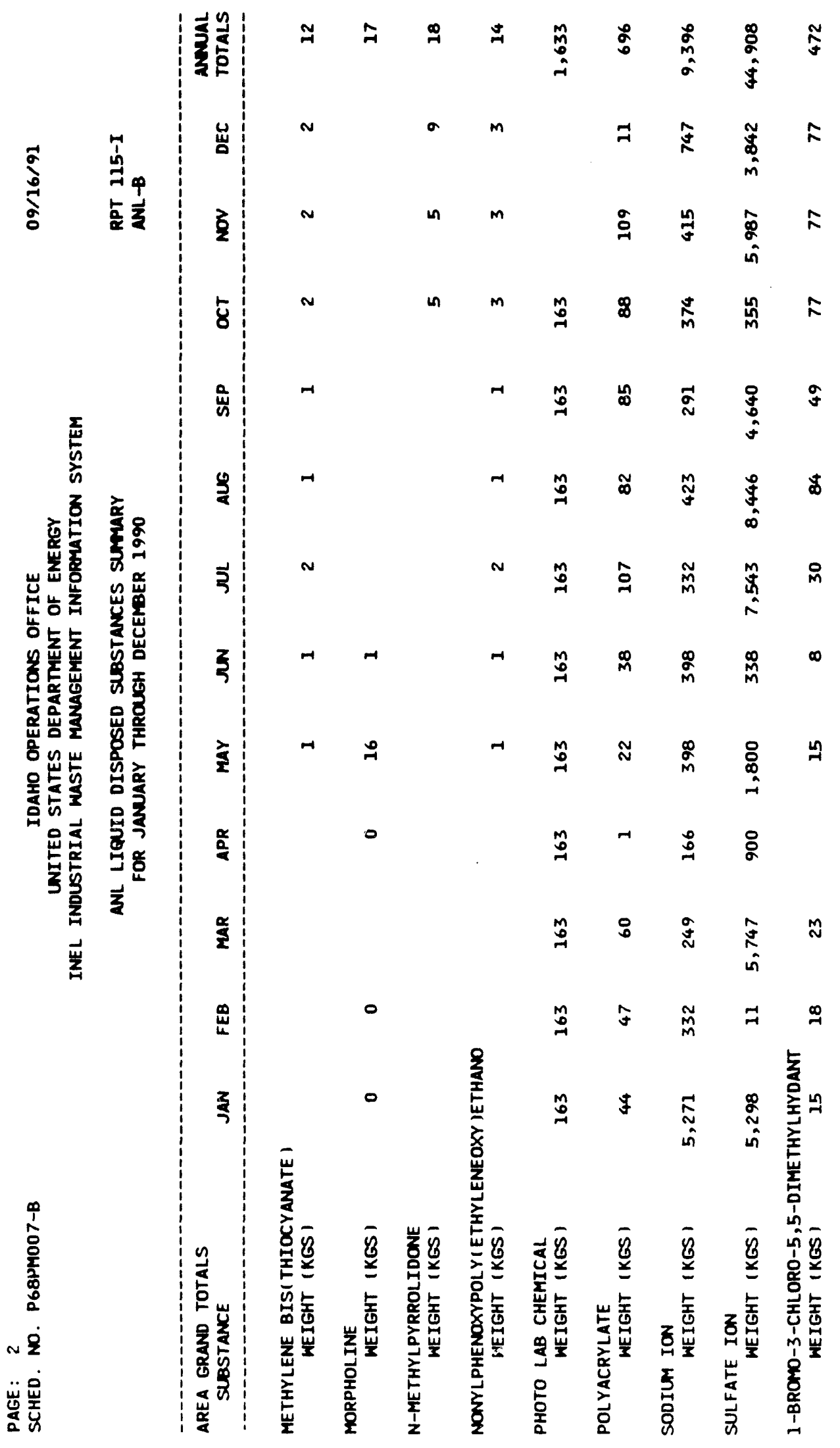

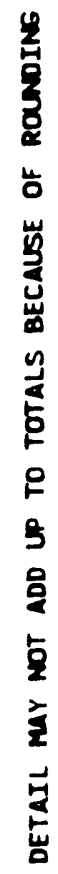




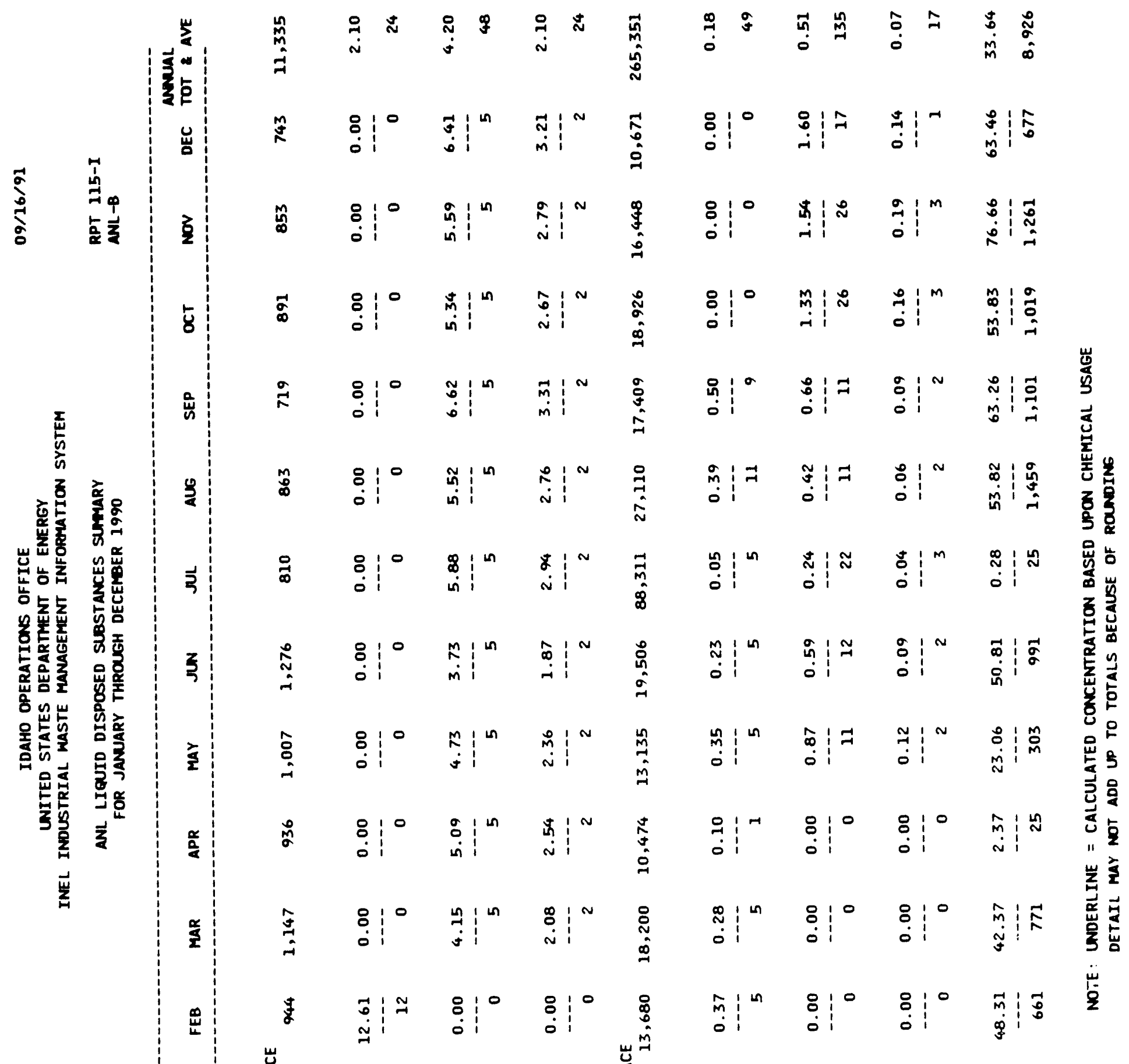

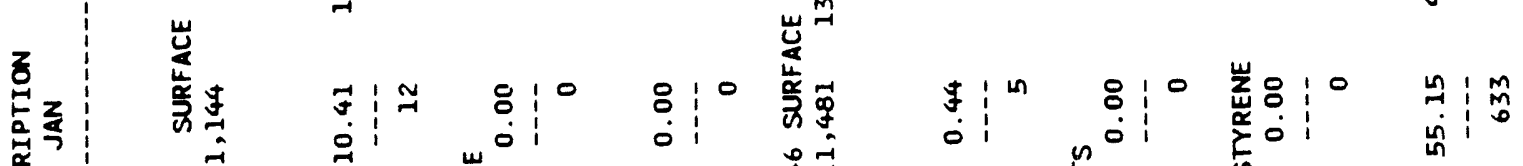

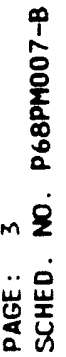




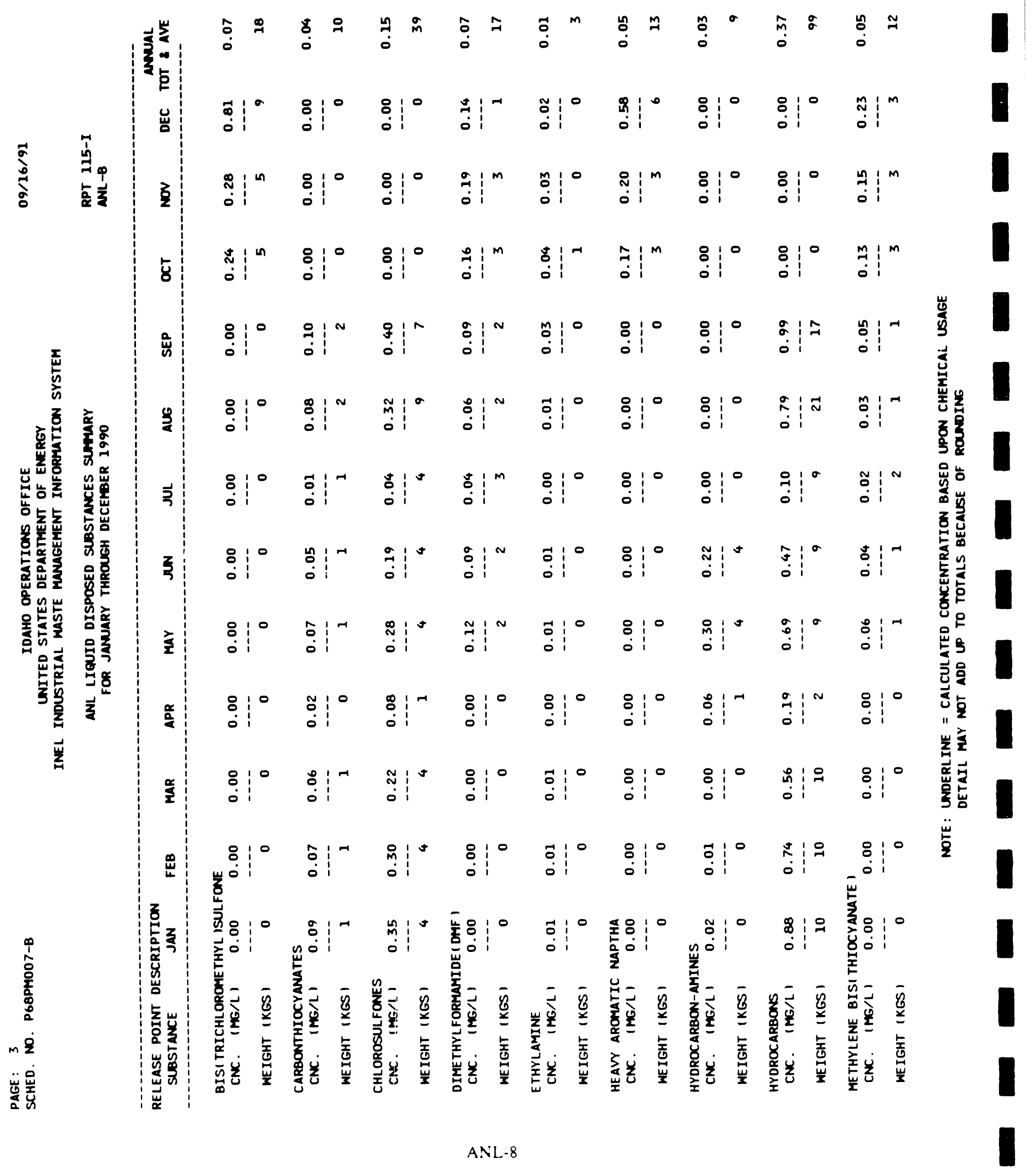


I

I

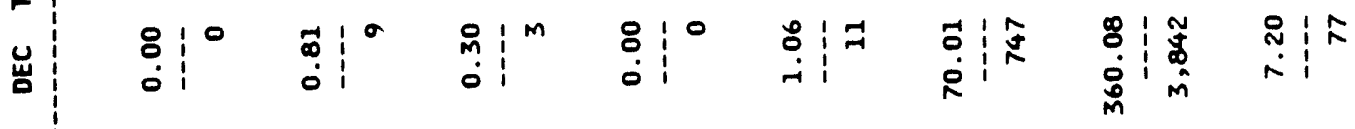

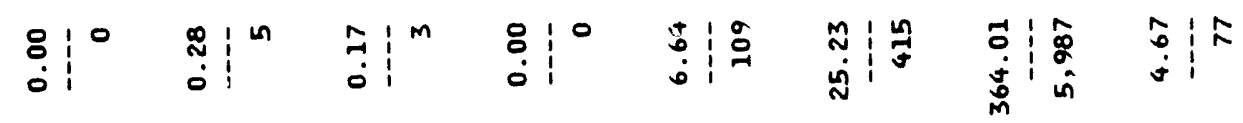

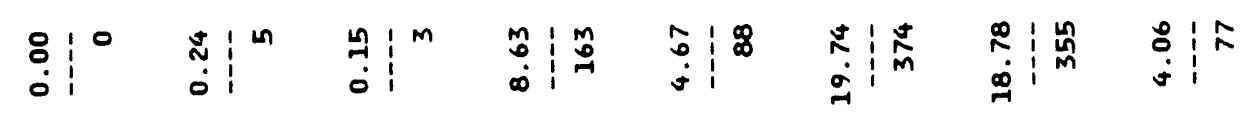

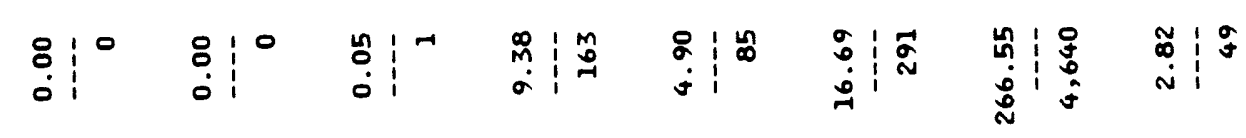

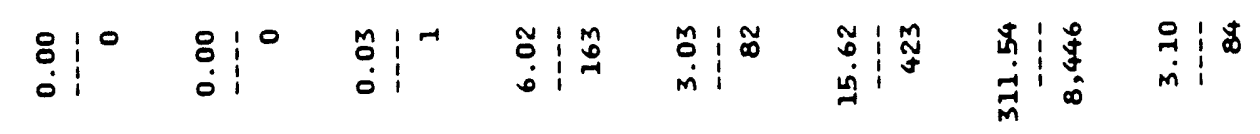

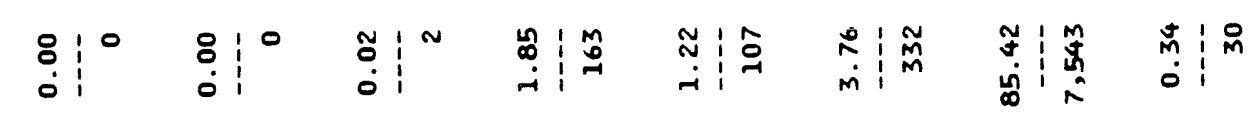

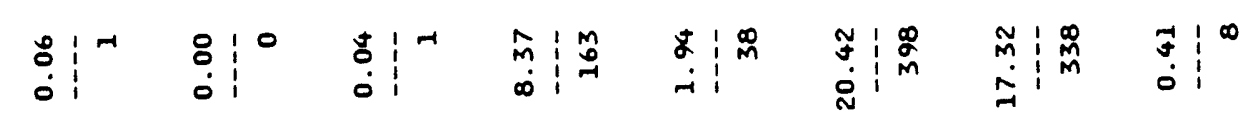

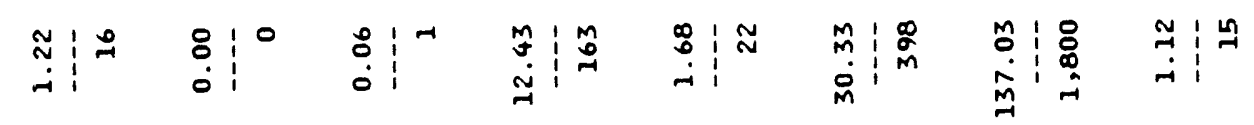

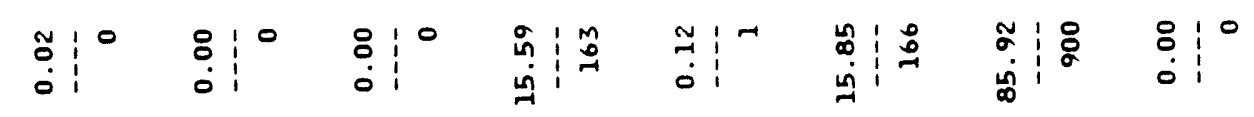

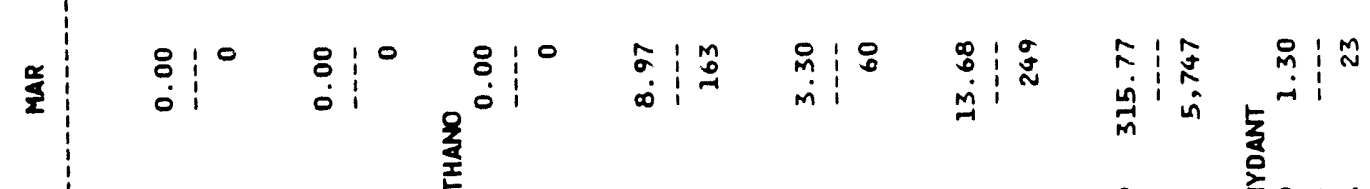

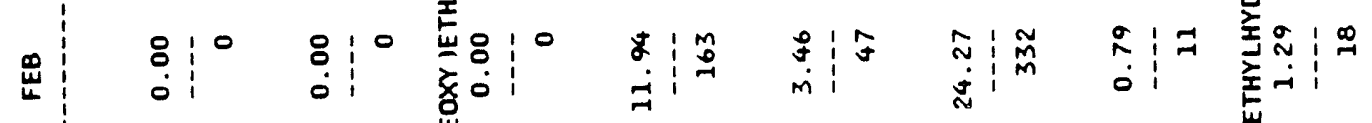

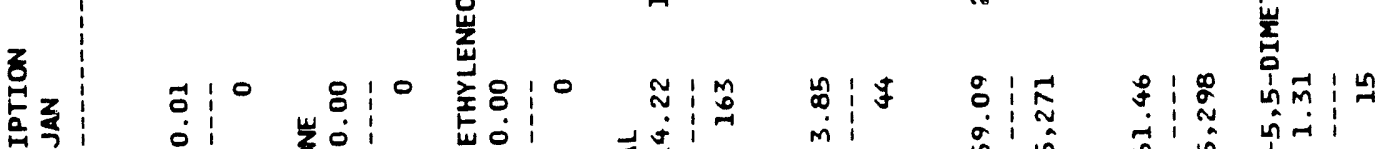




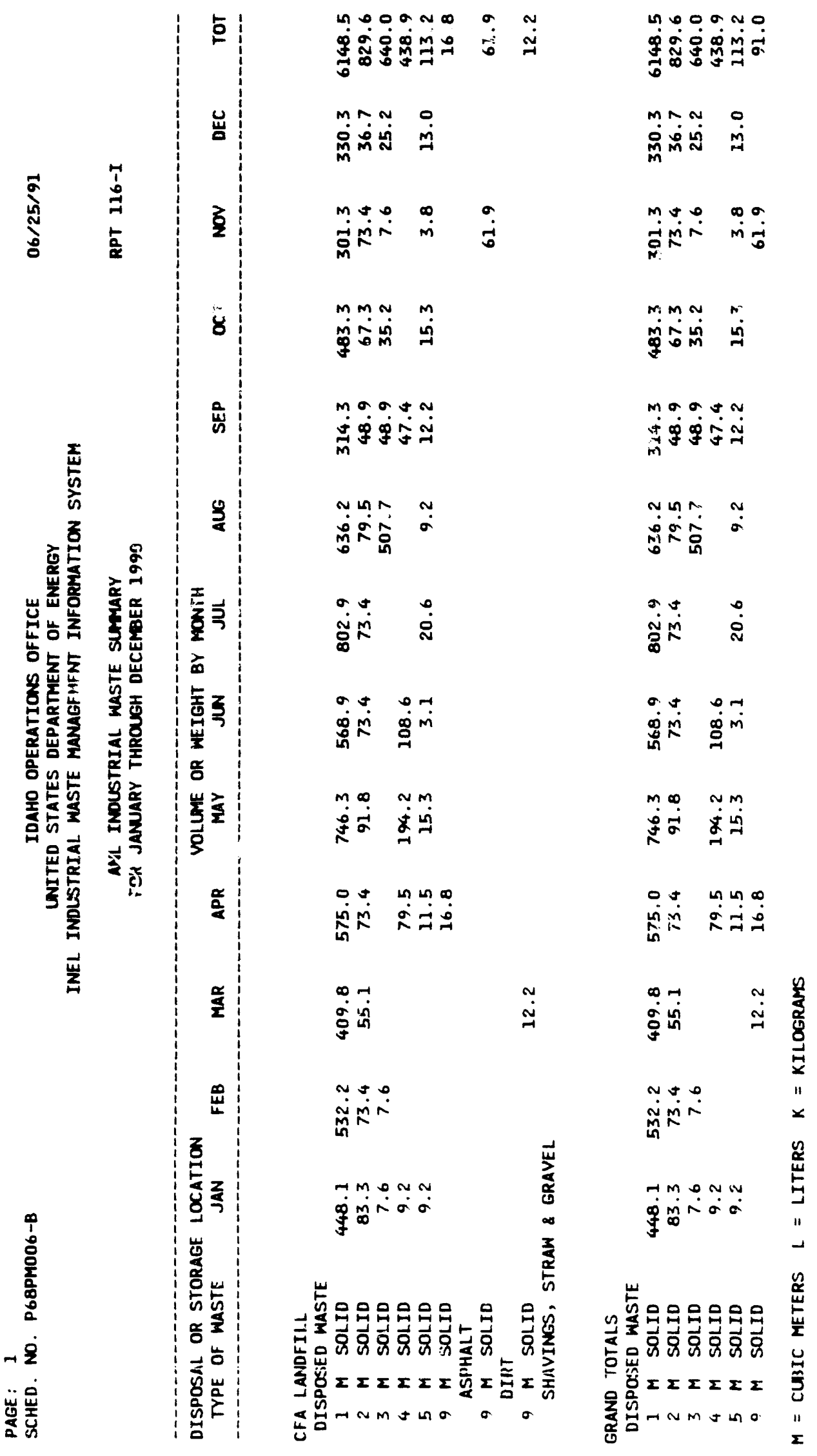




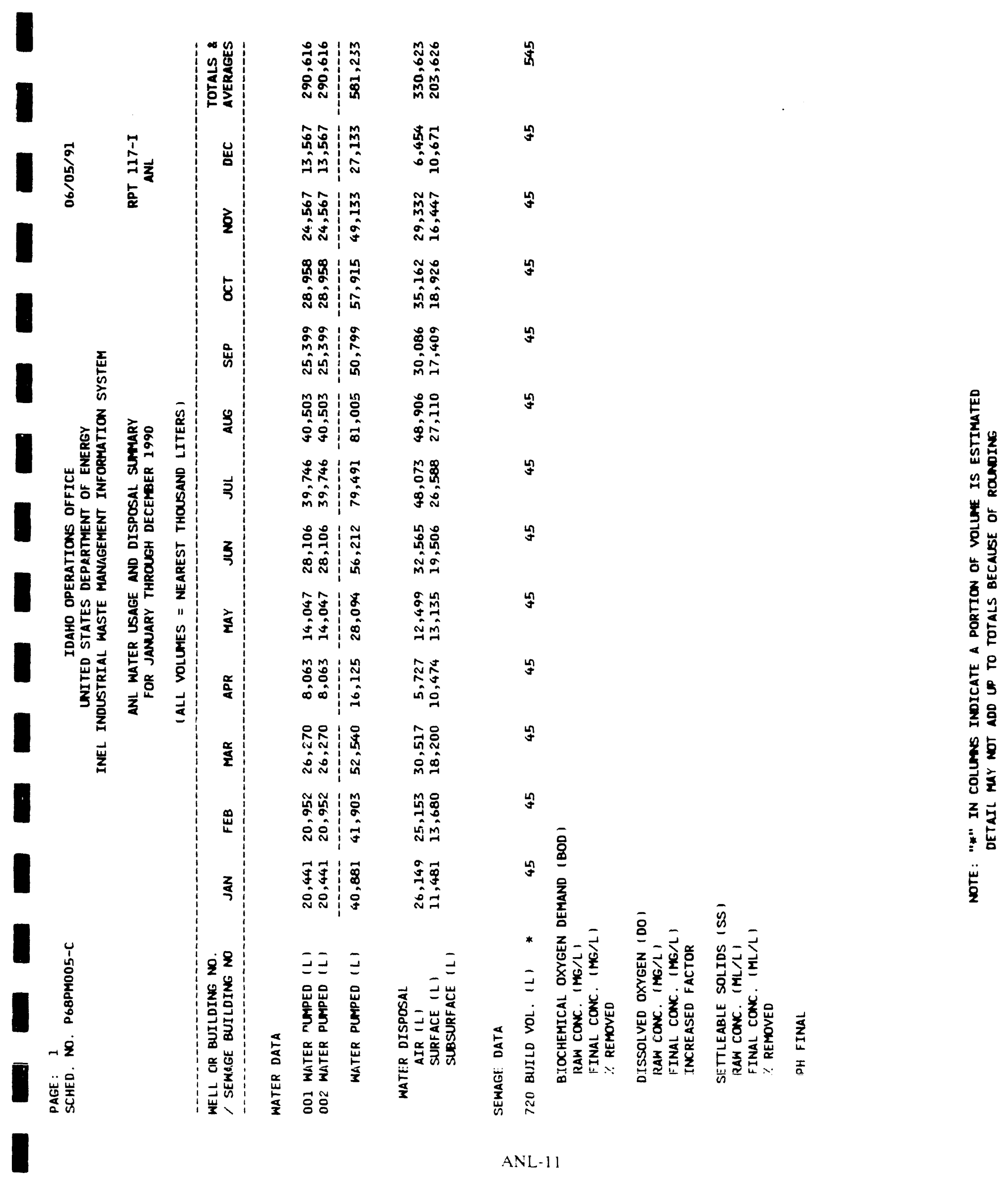



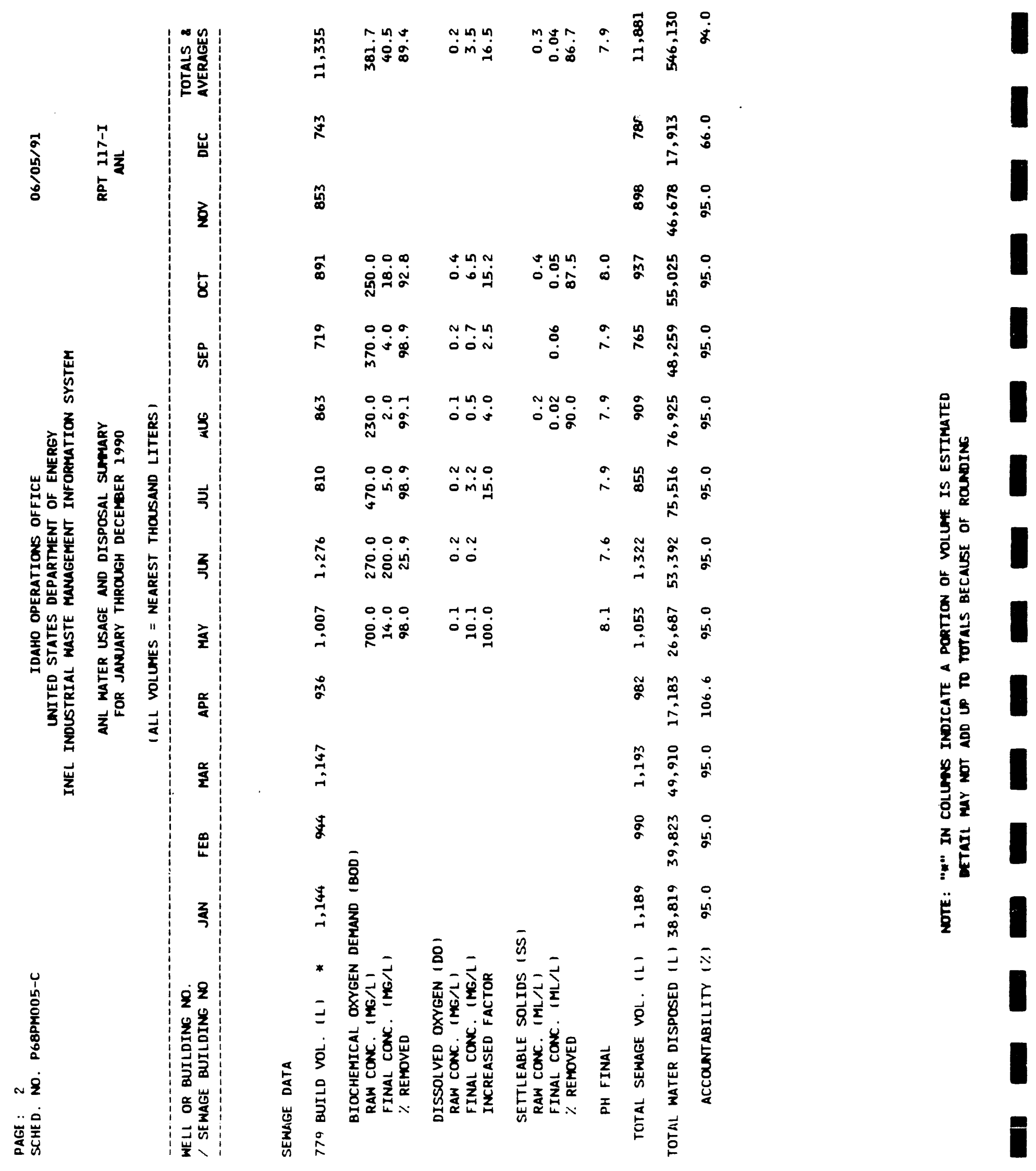


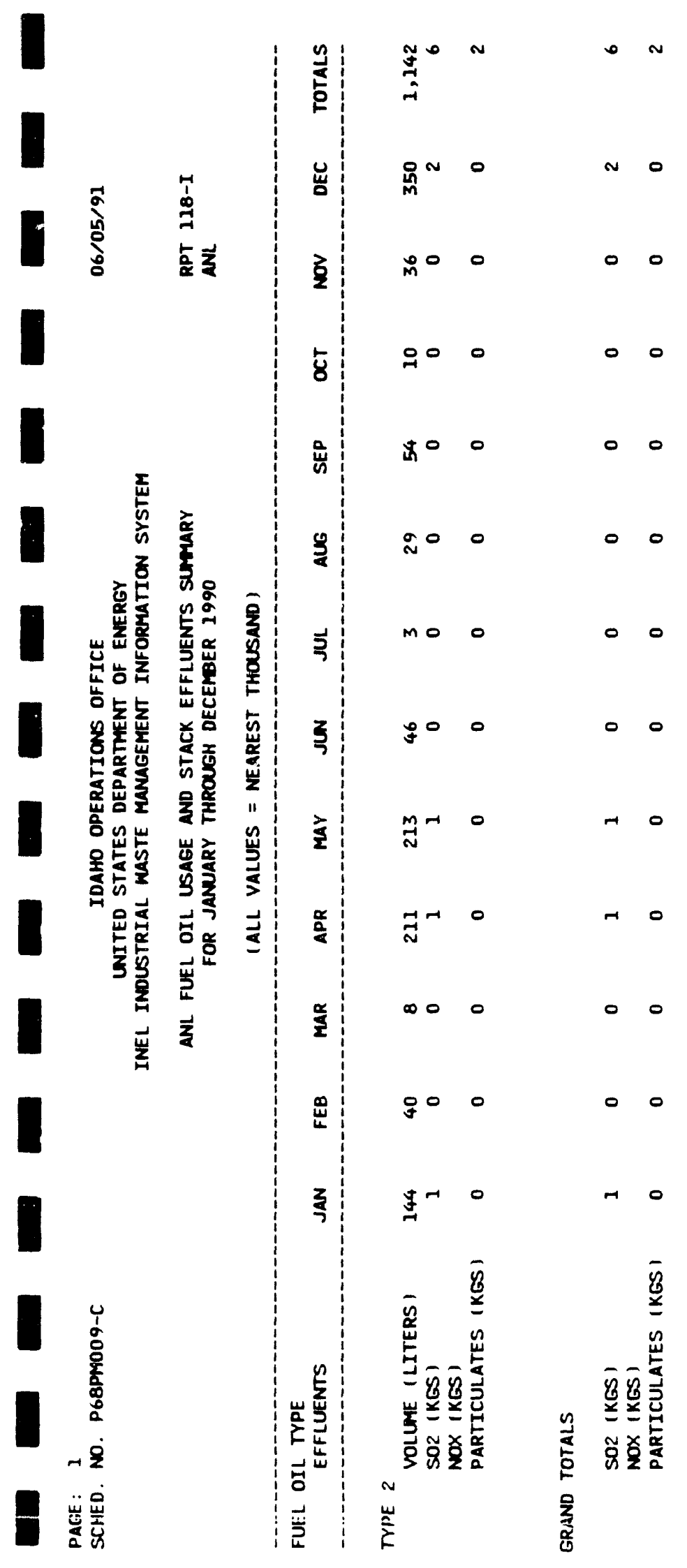


CENTRAL FACILITIES AREA, 1990 DETAIL DATA

Report 135 - CFA Liquid Disposed Substances Summary $\ldots \ldots \ldots \ldots \ldots$ CFA-3

Report 136 - CFA Industrial Waste Summary $\ldots \ldots \ldots \ldots \ldots \ldots \ldots \ldots \ldots \ldots \ldots$ CFA -8

Report 137 - CFA Water Usage and Disposal Summary ............... CFA-10

Report 138 - CFA Fuel Oil Usage and Stack Effluents Summary . . . . . . . . . CFA-11 
I

I

售:

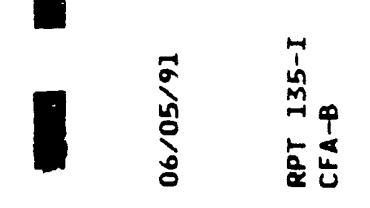

I

1

1

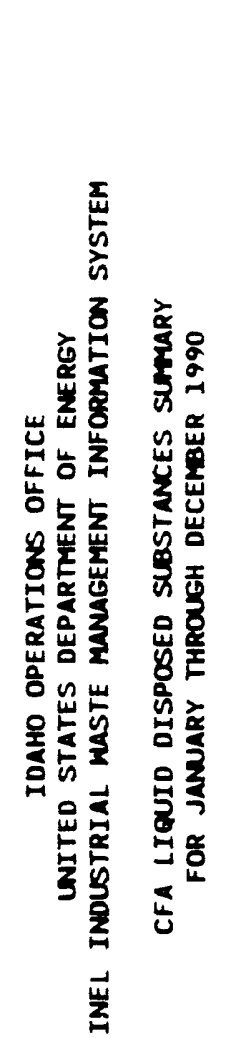

岁

n

I

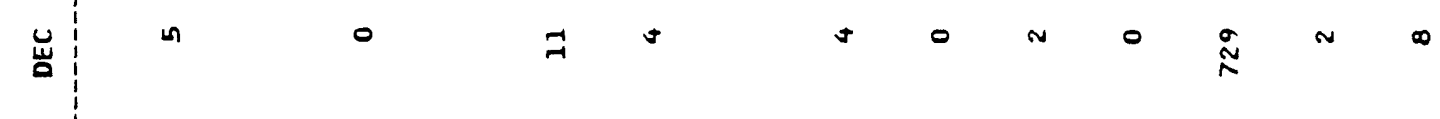

$2:$ n

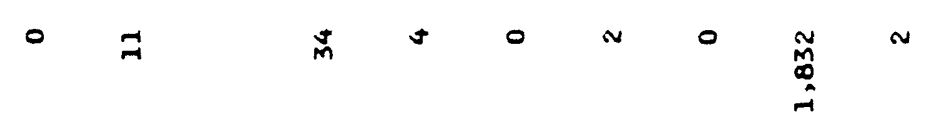

$\approx$

\begin{tabular}{l:ll} 
& in \\
\hline & &
\end{tabular}

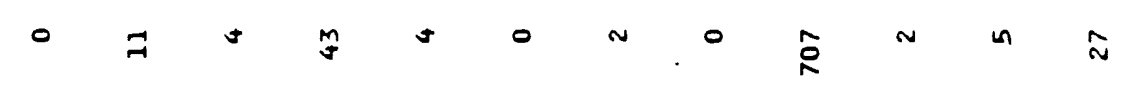

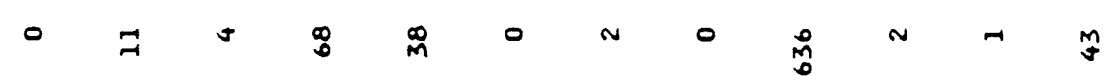

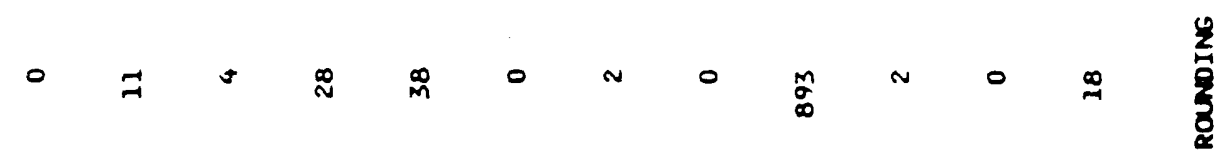

- a

事

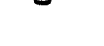

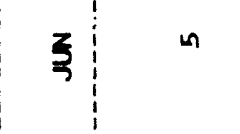

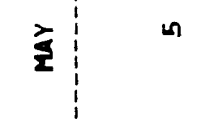

$\circ$
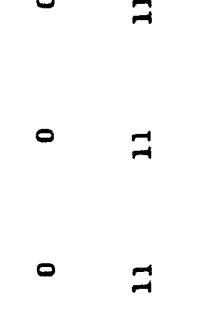

$\frac{\alpha}{2}$

$\frac{2}{2}$

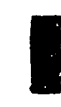

思

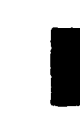

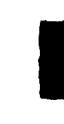

$\frac{1}{4}$

$-\dot{2}$

】

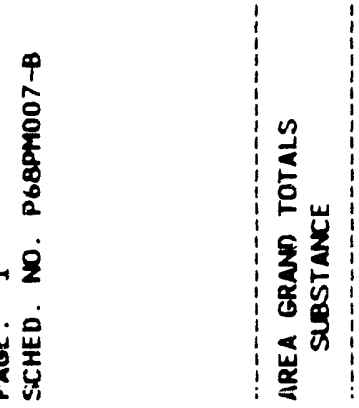

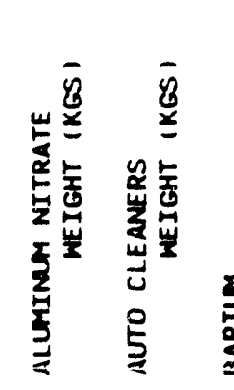

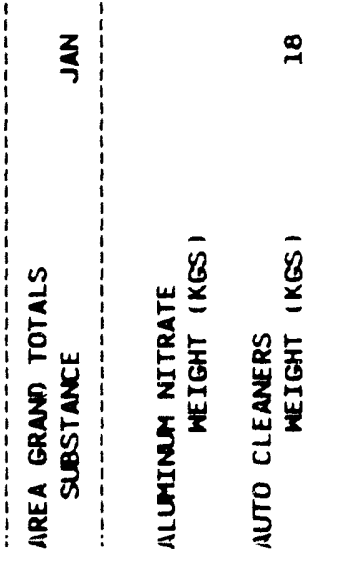

$\Phi$ 


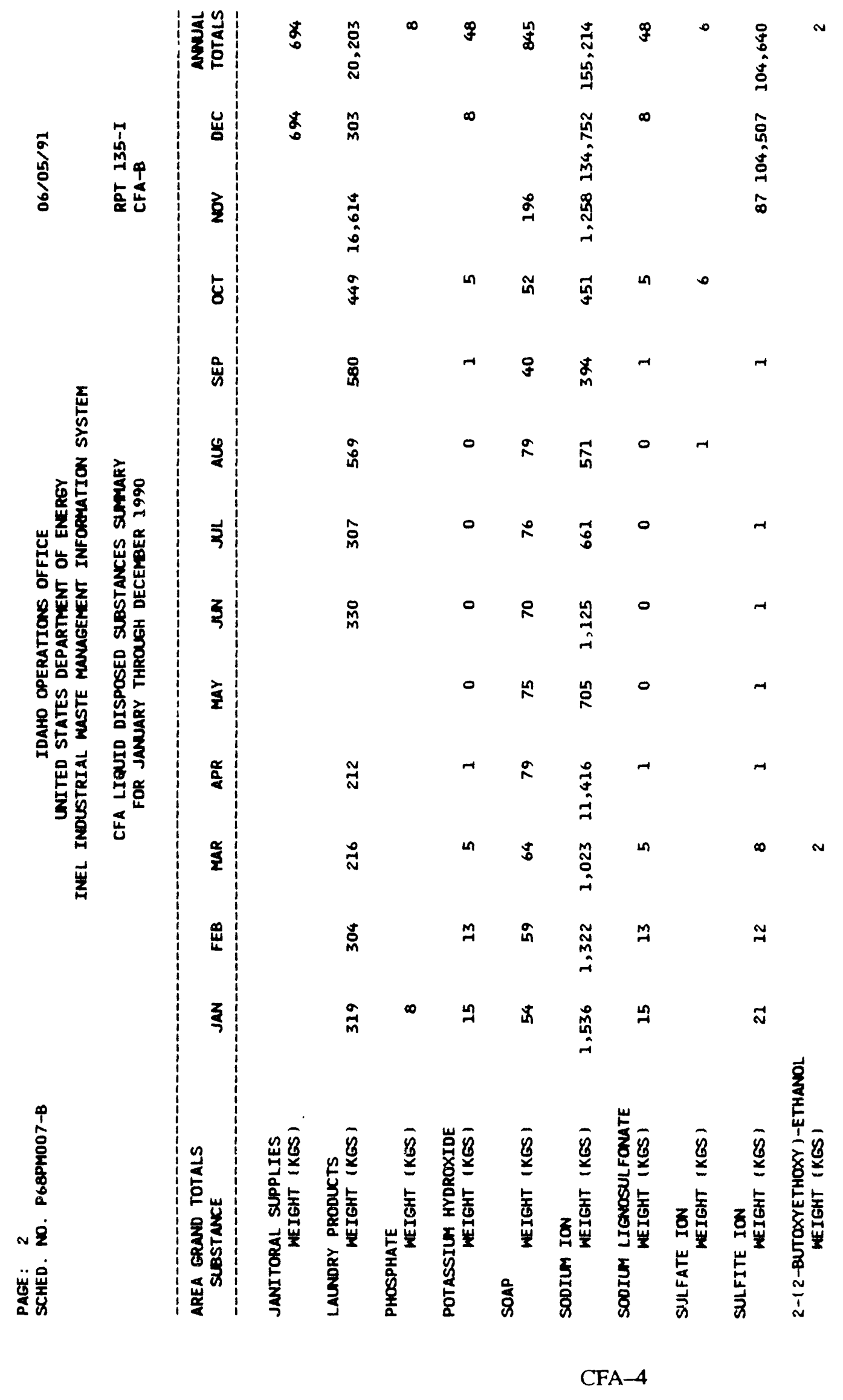

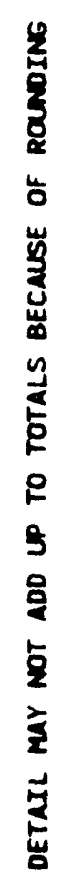


】

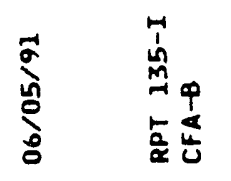

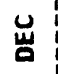

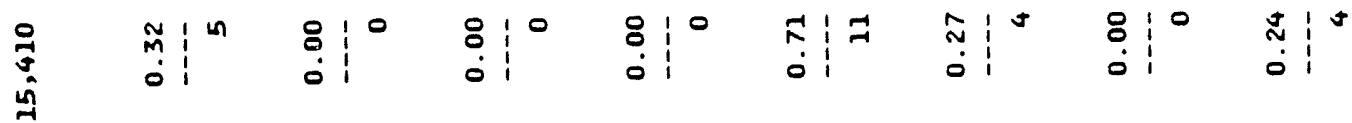

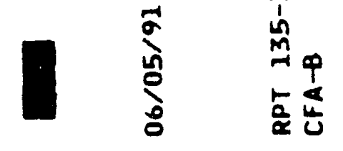

3

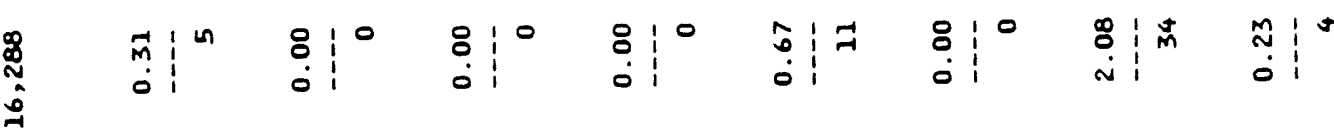

$8)$

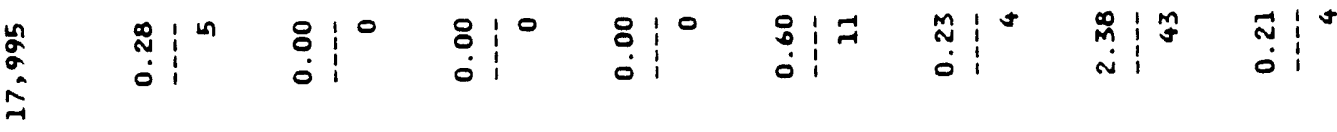

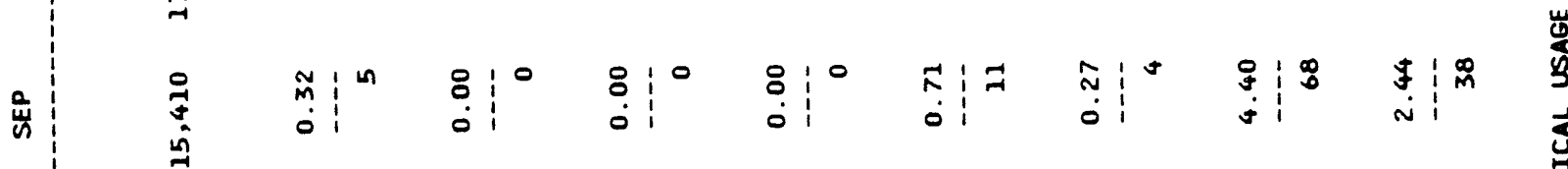

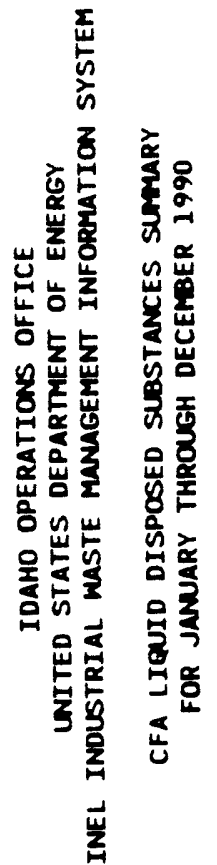

3

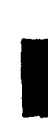

$\stackrel{1}{:}$

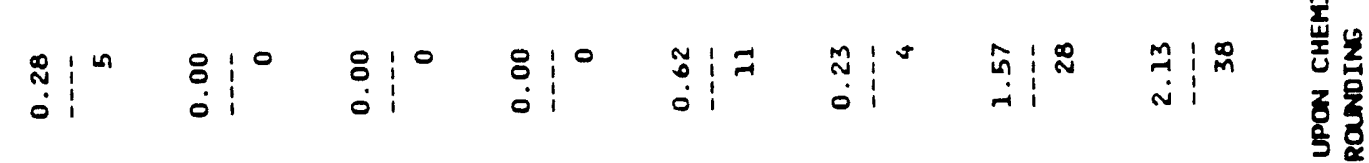

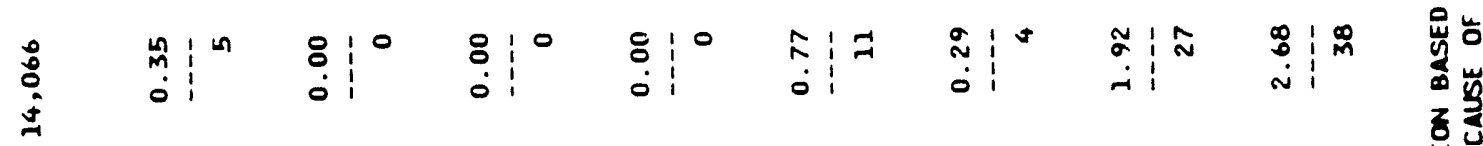

₹ $\quad$ :

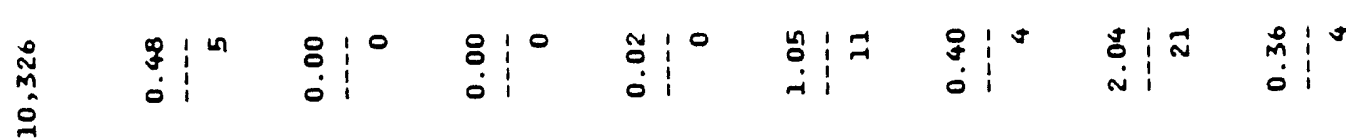

3

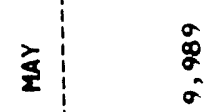

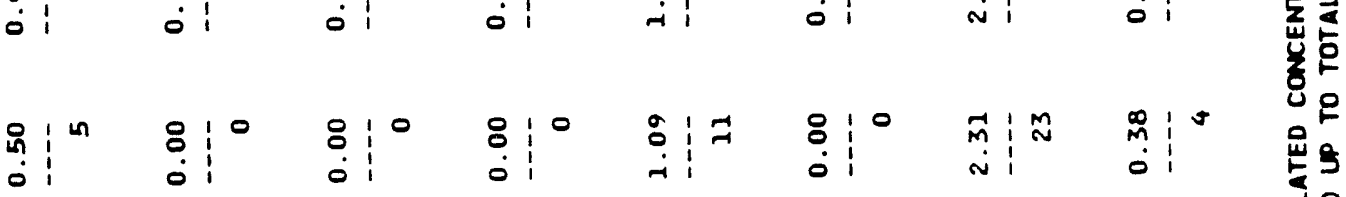

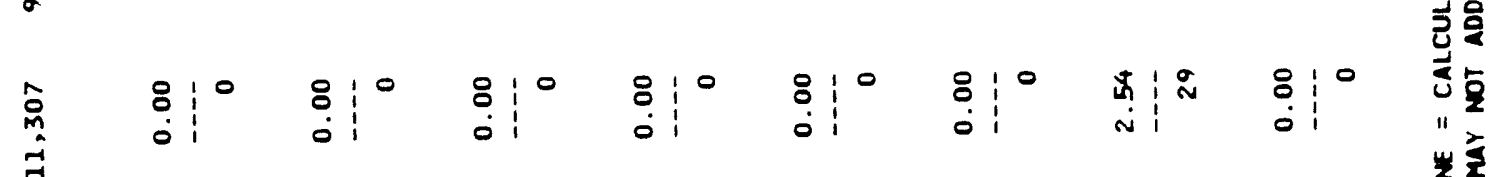

$\frac{\alpha}{2}$

$\frac{2}{2}$

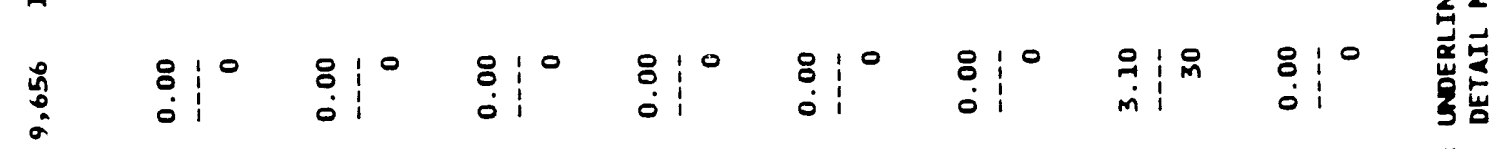

蚛

压予

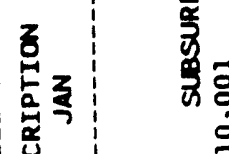

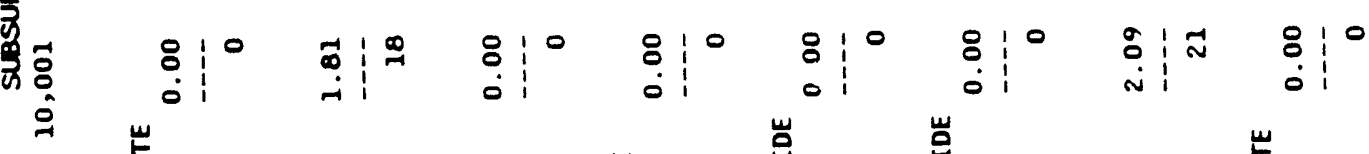

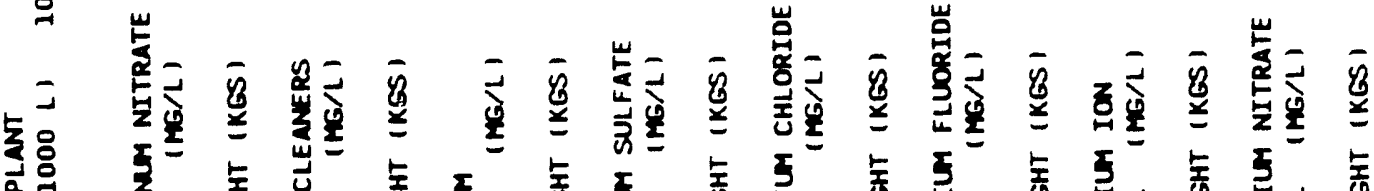

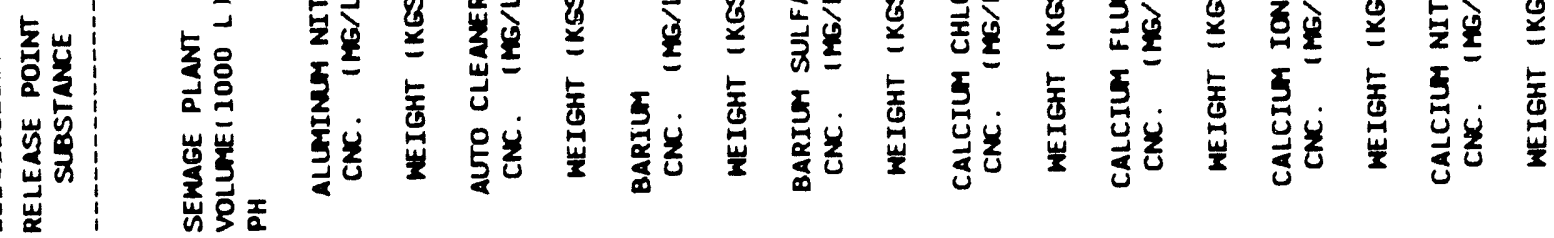

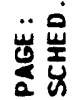




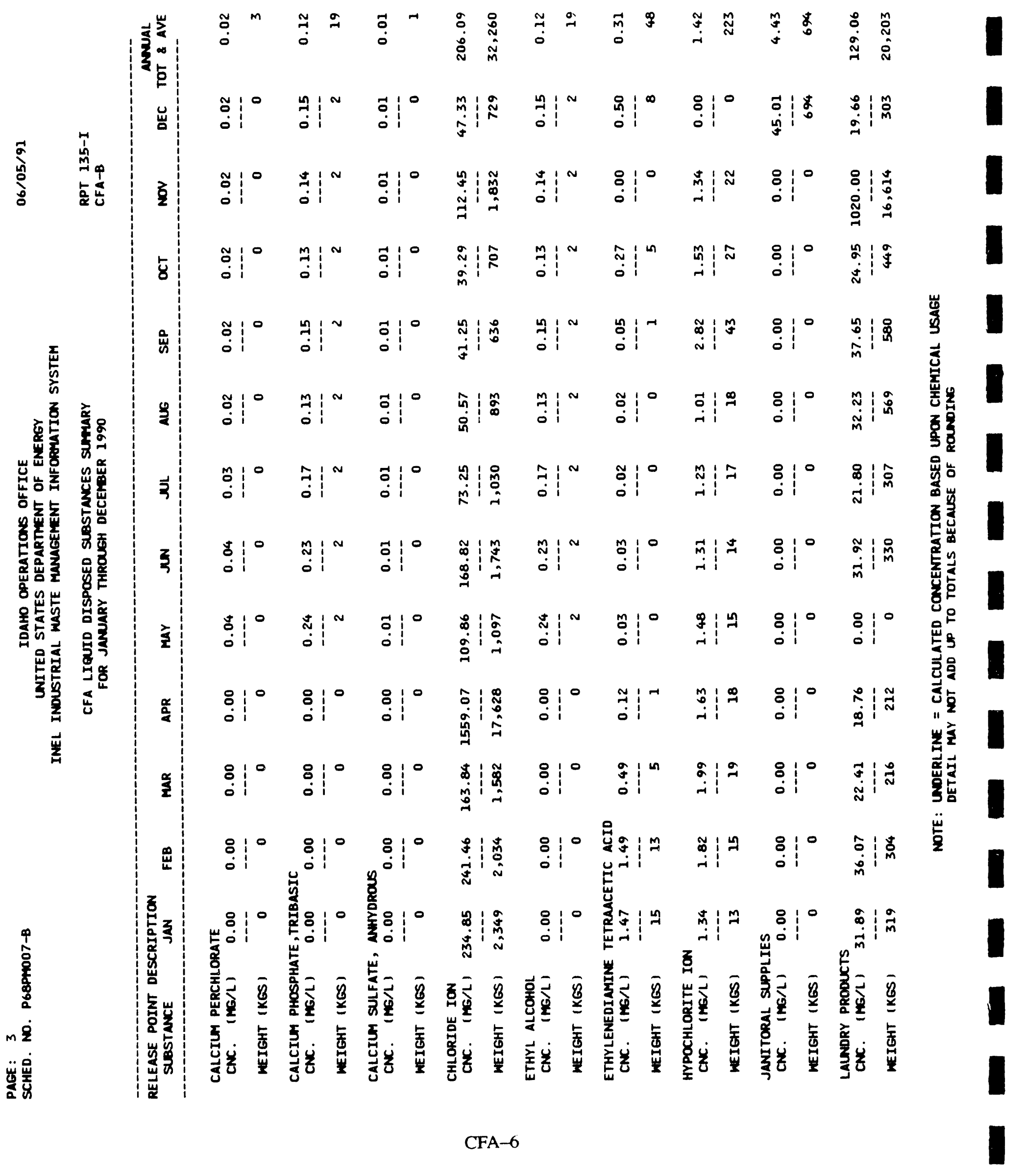




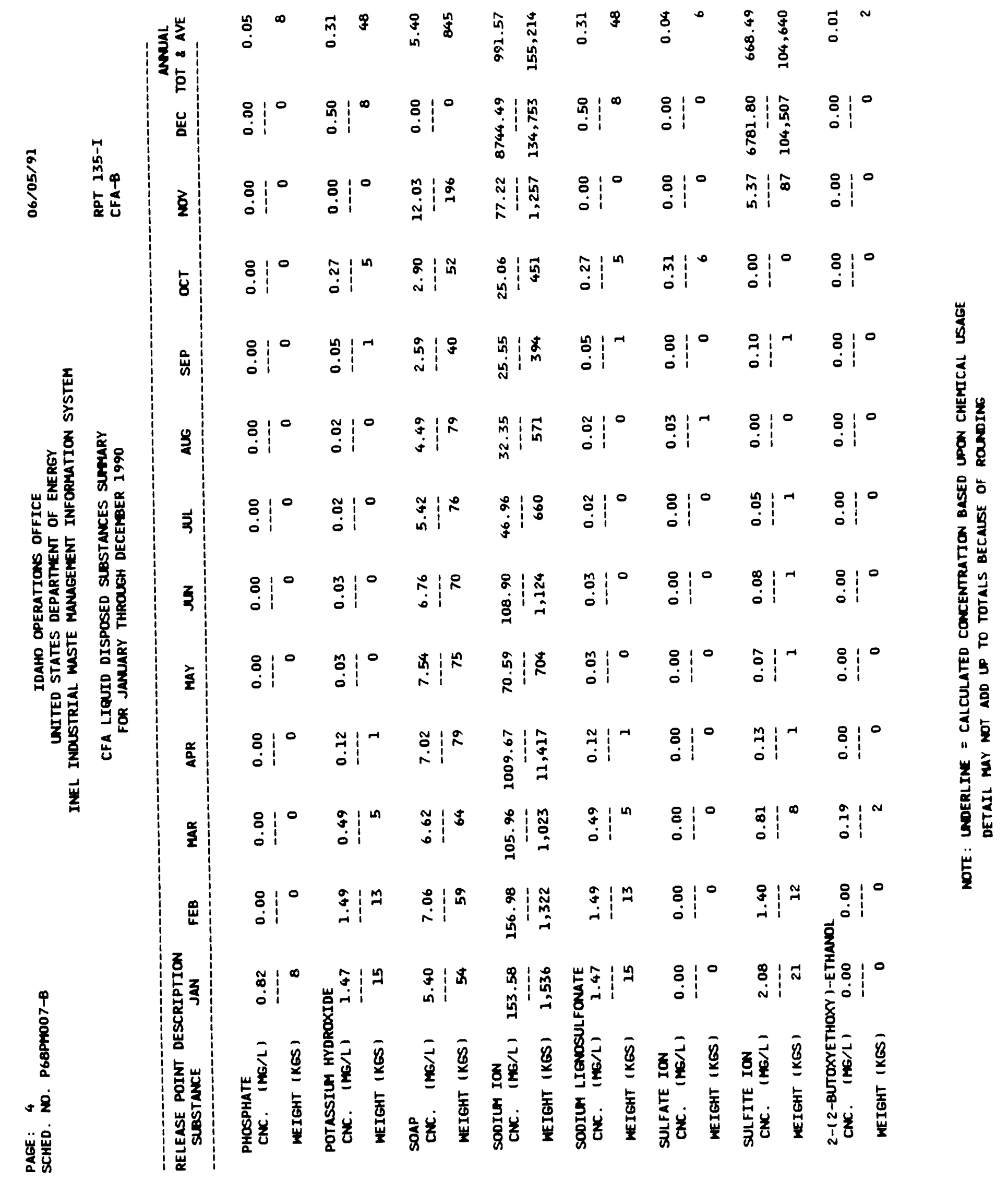




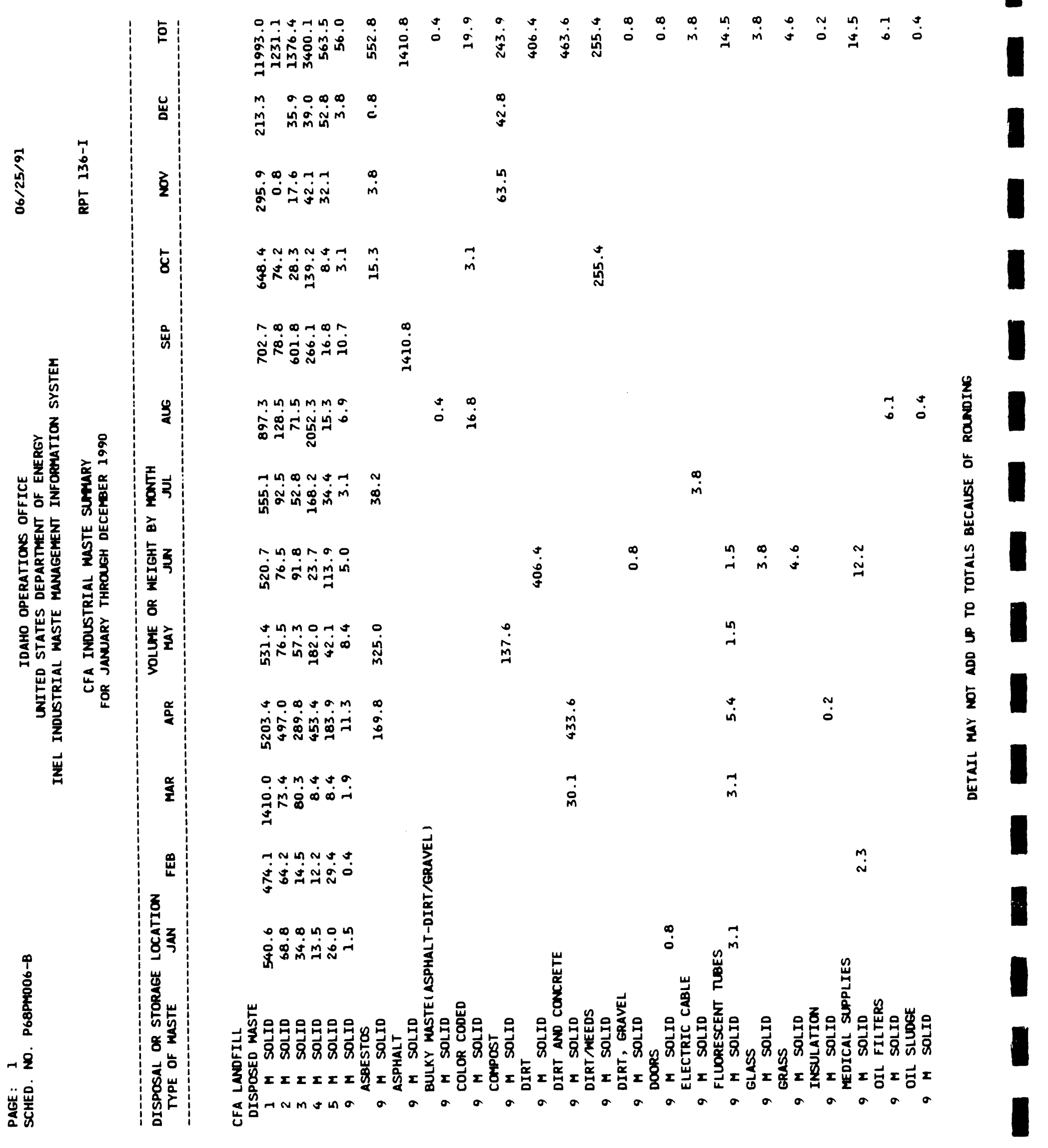




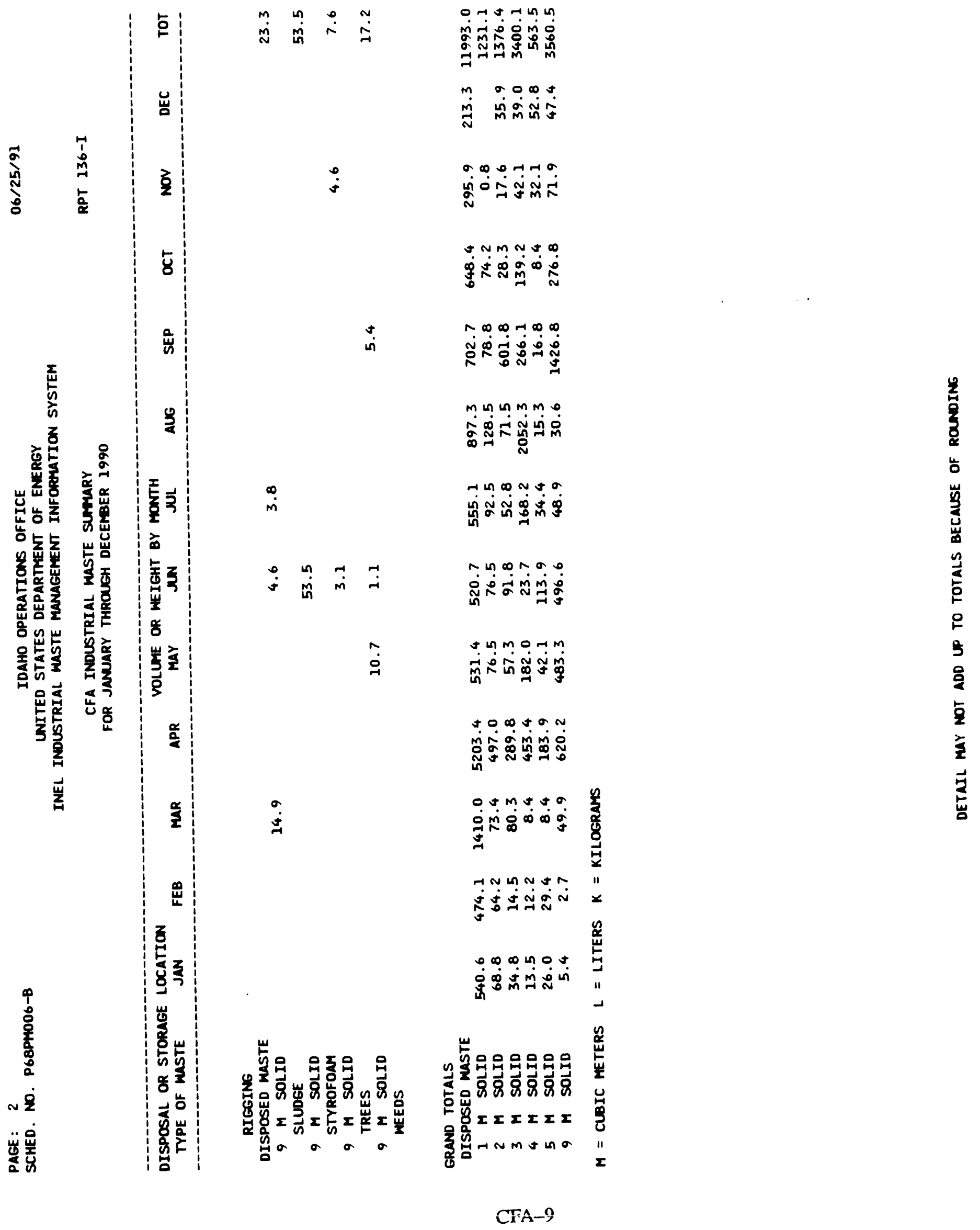




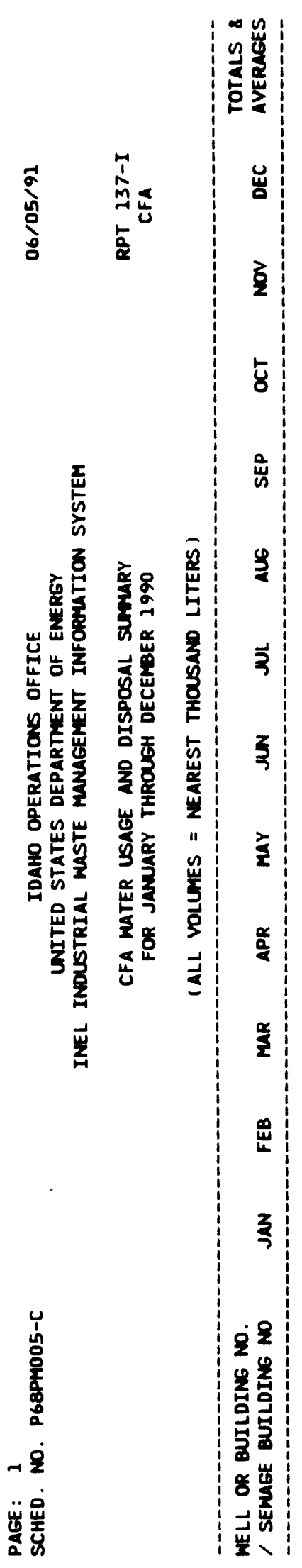

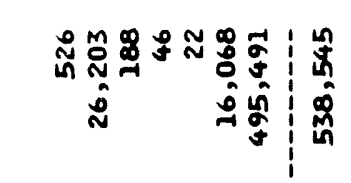

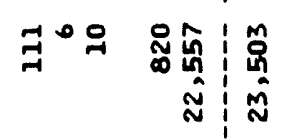

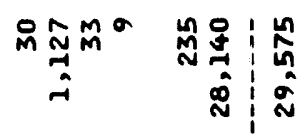

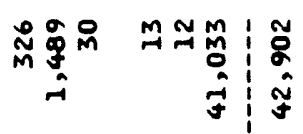

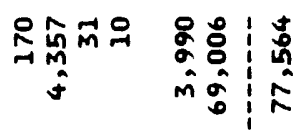

号

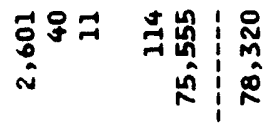

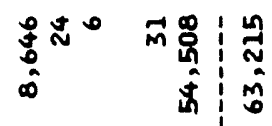

$\underset{m}{m}$

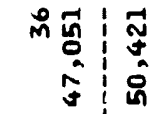

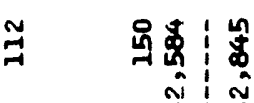

出

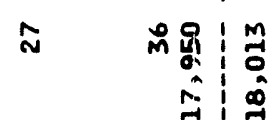

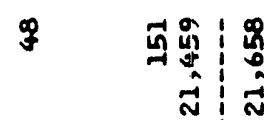

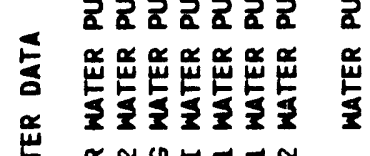

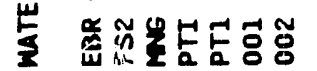

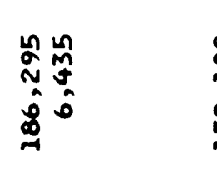

串 ํำ

เัช

(2)

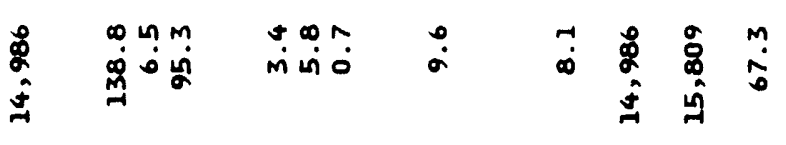

जी

ตุบิ

$\stackrel{\circ}{\stackrel{s}{s}}$

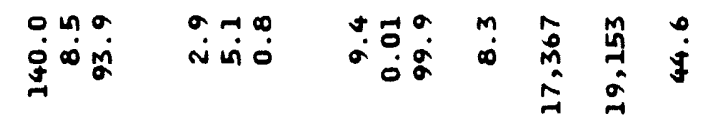

变

N

to

守

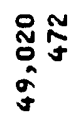

触

กั

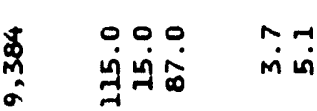

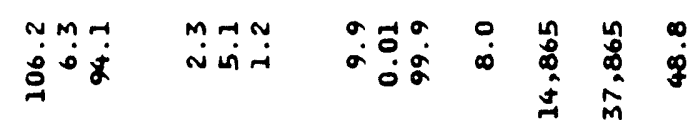

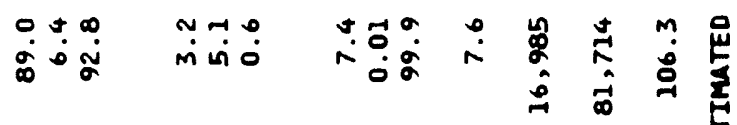

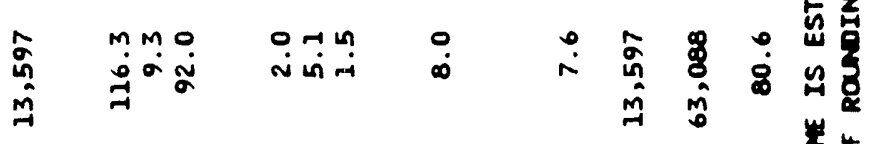

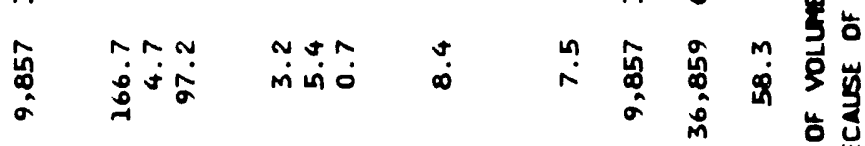

व न्रक्ष

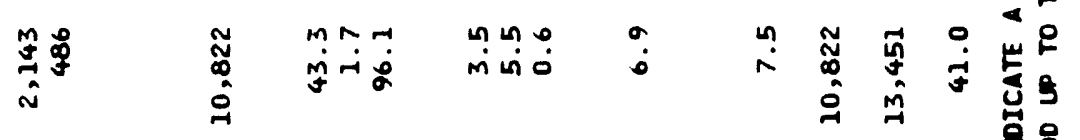

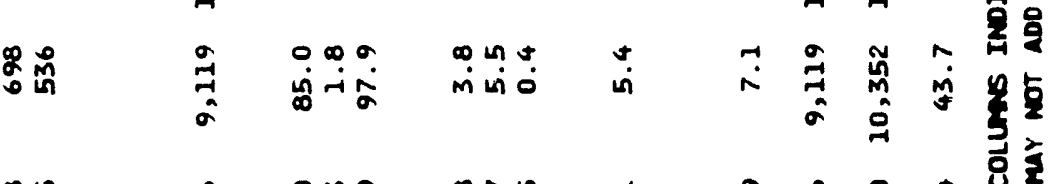

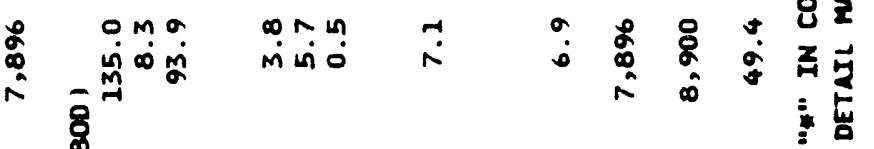

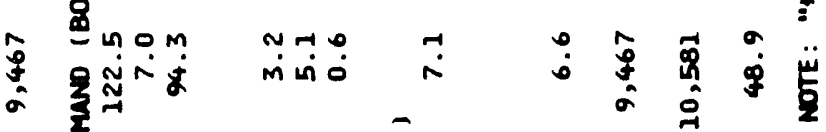

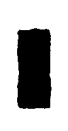

总蛋

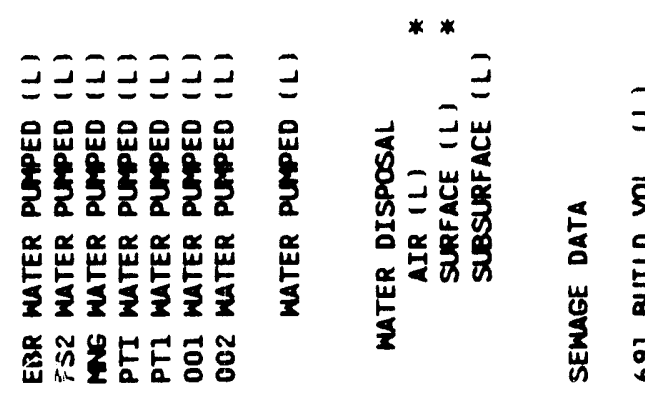

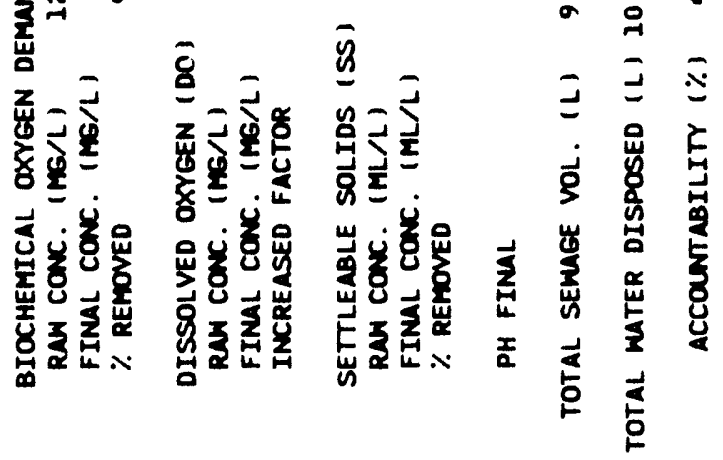


I

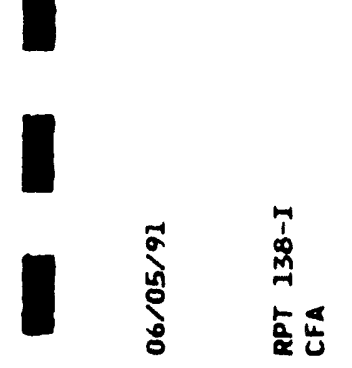

最

I

$\stackrel{\infty}{\sim} \quad \overbrace{}^{m}$

No ño

No ño

3) श० กू०

5000

I

I

站

I

崫 N० $^{\circ}$

○

F०

3) no no no

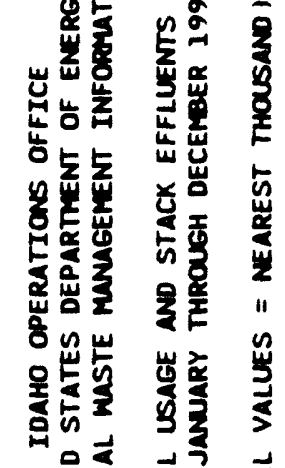

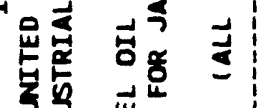

3 - 300

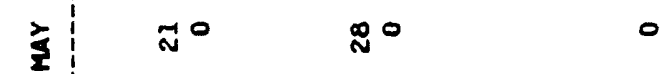

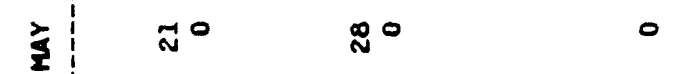

$m_{0} \quad m^{\circ} \quad$

a

90 9० $^{\circ}$ o

䒠

嵌

蛋

No

3 mo

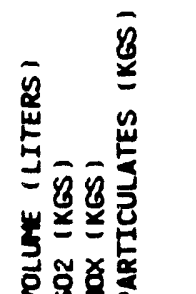

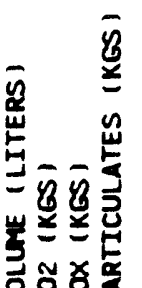

我藏登

崫

崩

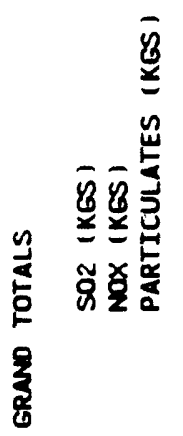




\section{CHEMICAL PROCESSING PLANT, 1990 DETAIL DATA}

Report 144 - CPP Airborne Disposed Substances Summary ........... CPP-3

Report 145 - CPP Liquid Disposed Substances Summary . . . . . . . . . . . CPP-5

Report $146-\mathrm{CPF}$ Industrial Waste Summary $\ldots \ldots \ldots \ldots \ldots \ldots \ldots \ldots \ldots$ CPP-7

Report 147 - CPP Wlater Usage and Disposal Summary ............ CPP-9

Report 148 - CPP Fuel Oil Usage and Stack Effluents Summary .......... CPP-10 


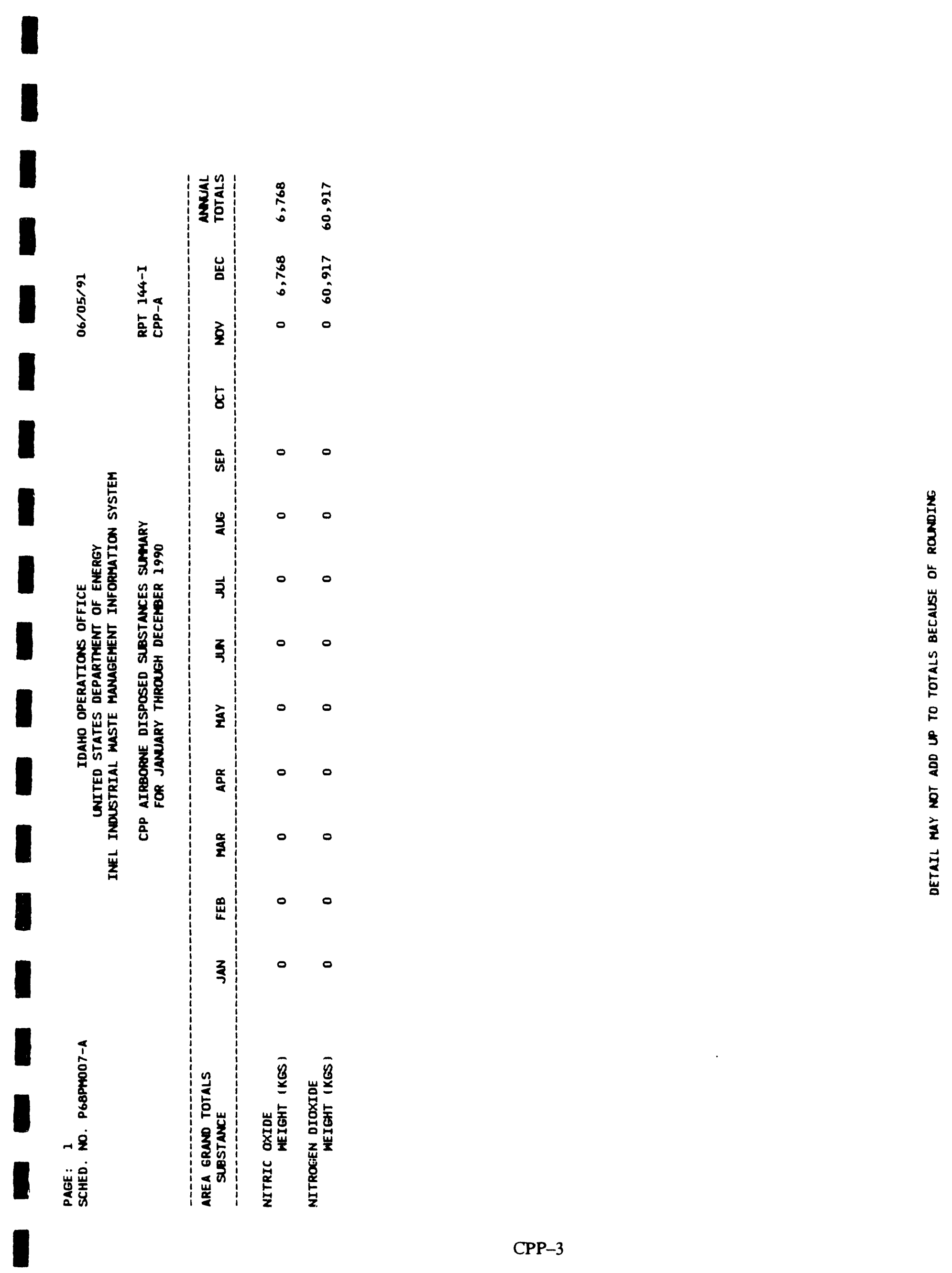



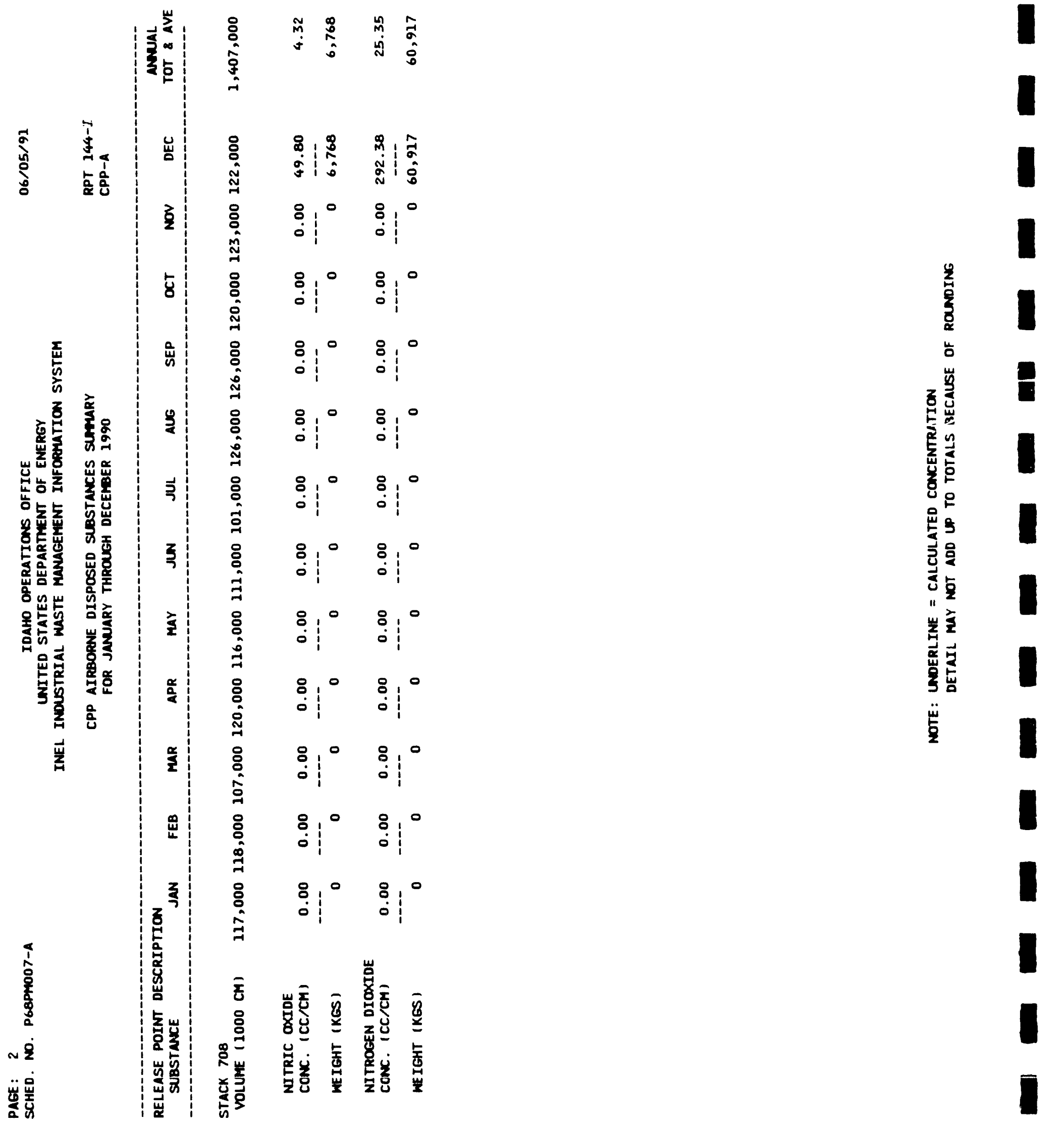


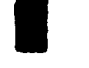

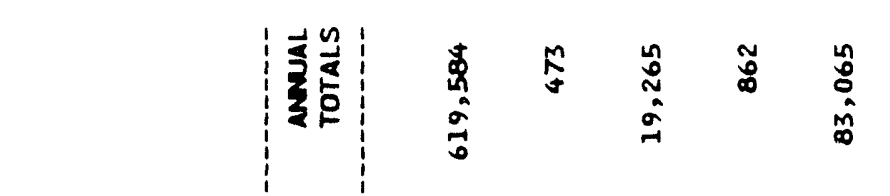

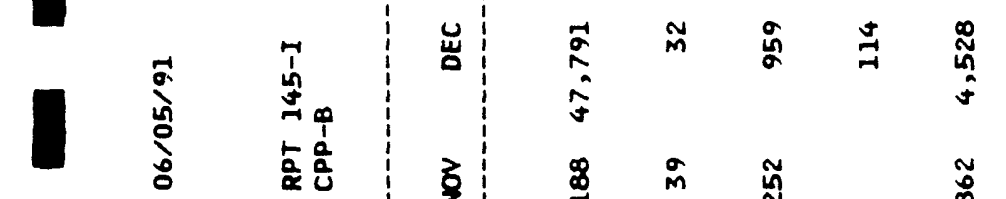

I

I

I

鹿

I

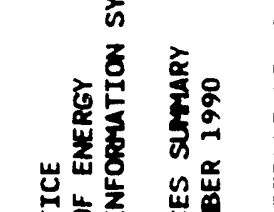

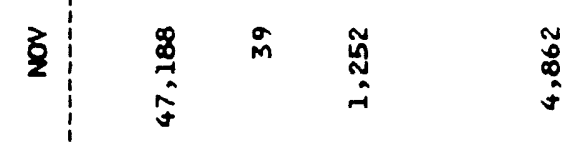

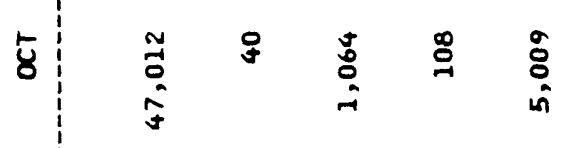

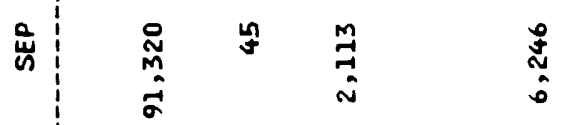

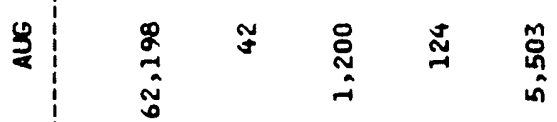

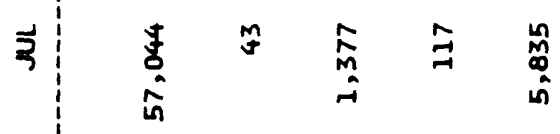

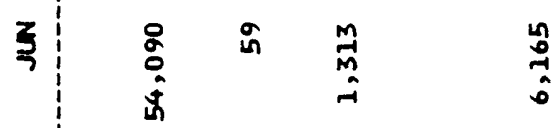

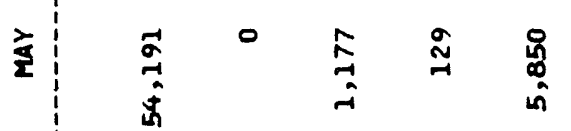

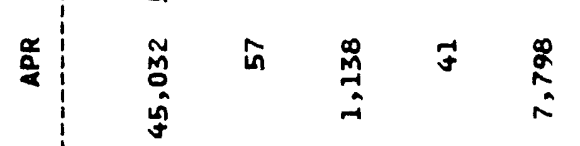

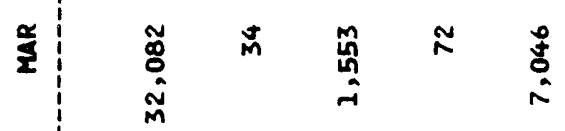

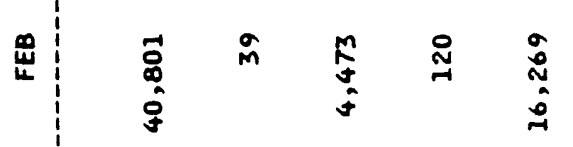

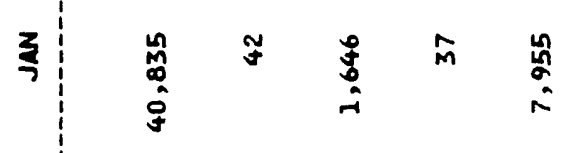

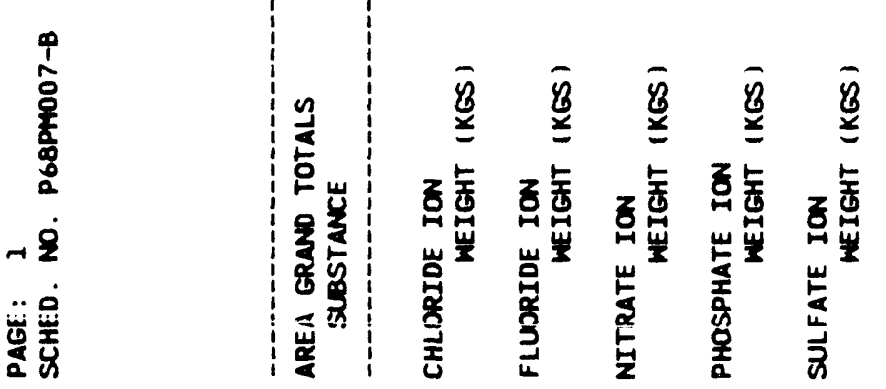



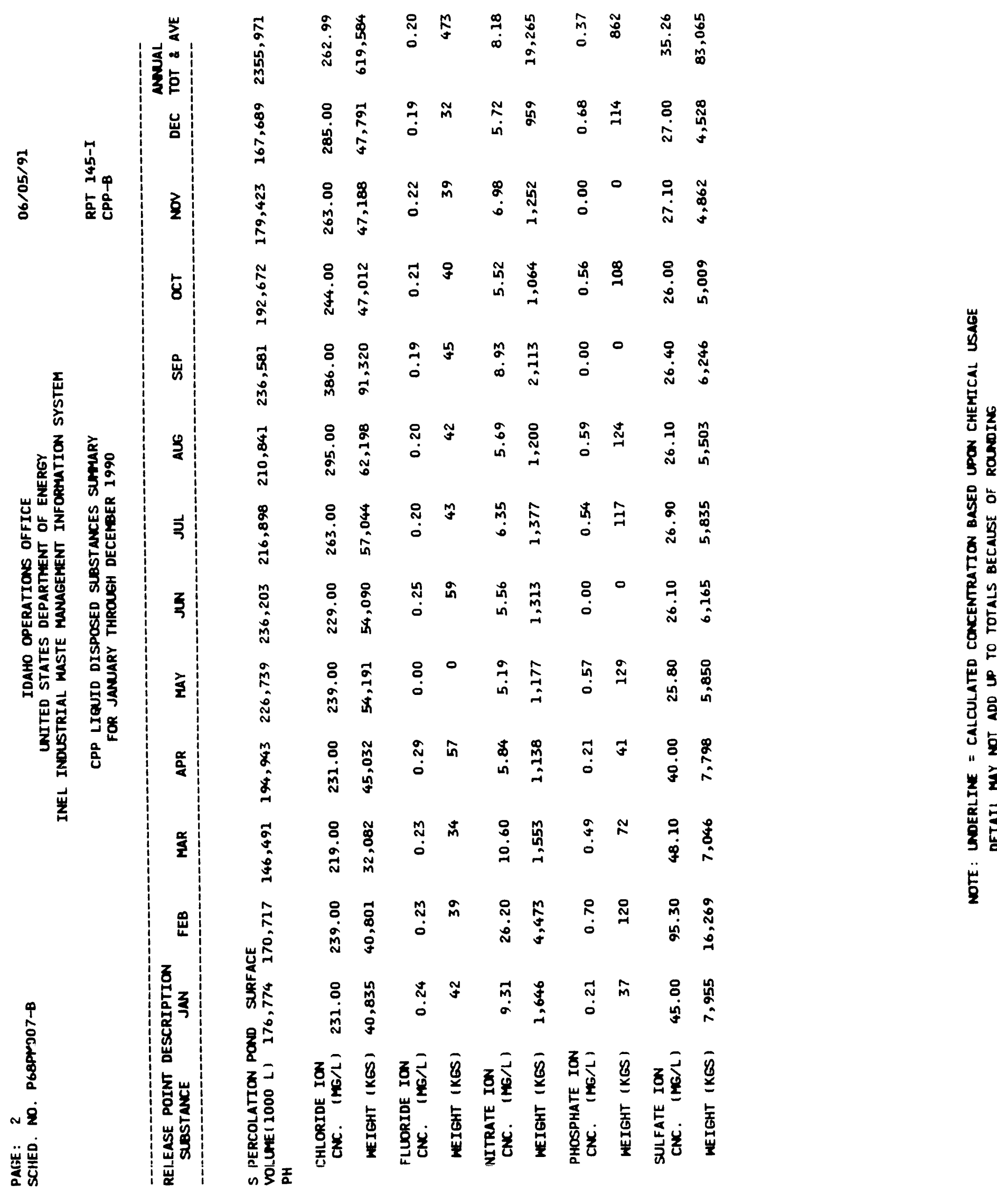
I

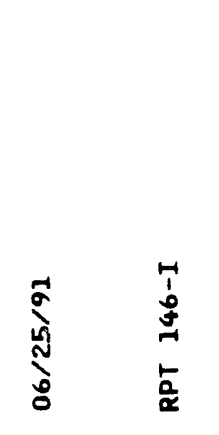

I

I

I

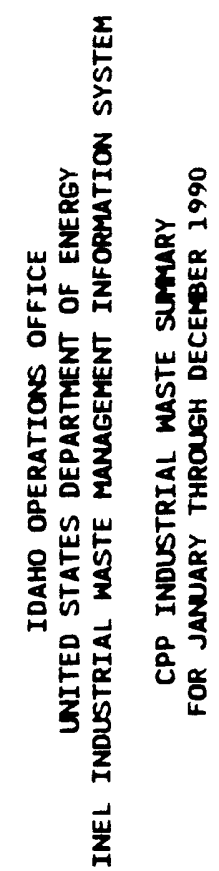

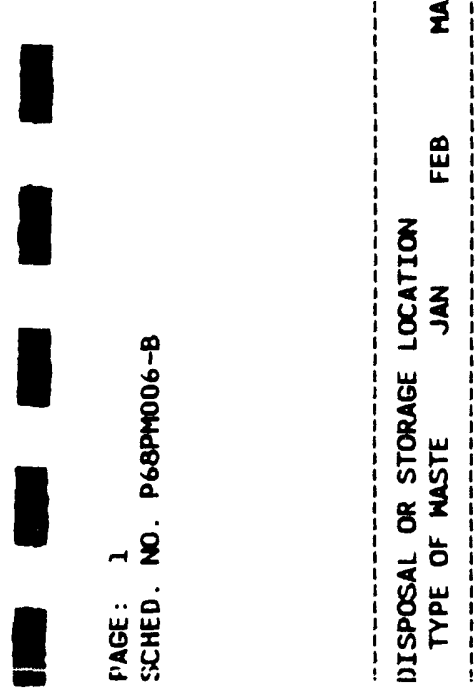

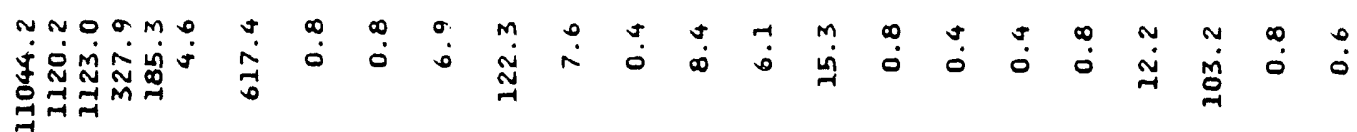

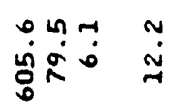

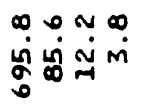

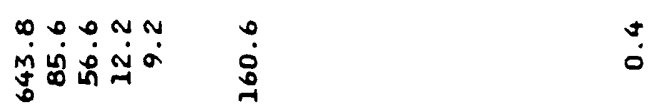

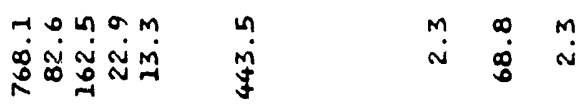

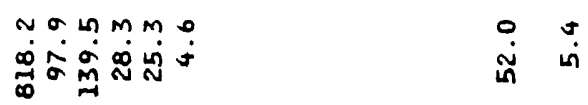

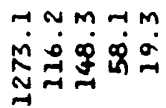

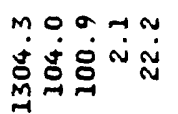

$\stackrel{\infty}{\circ}$

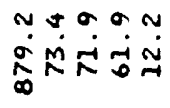

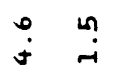

$\stackrel{4}{0}$

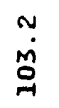

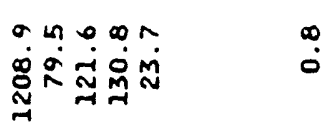

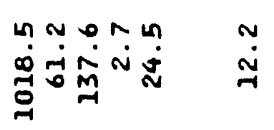

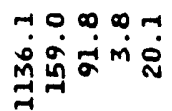

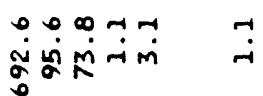

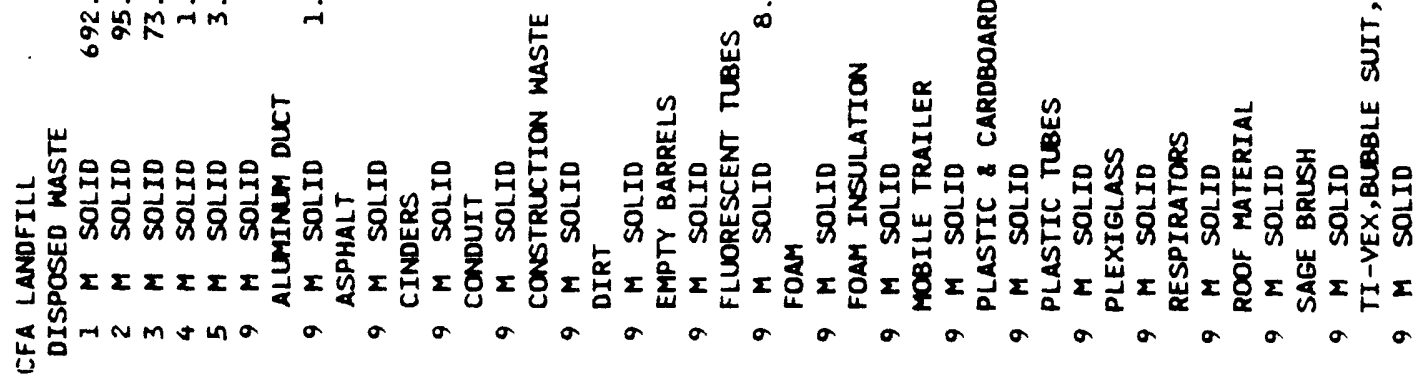



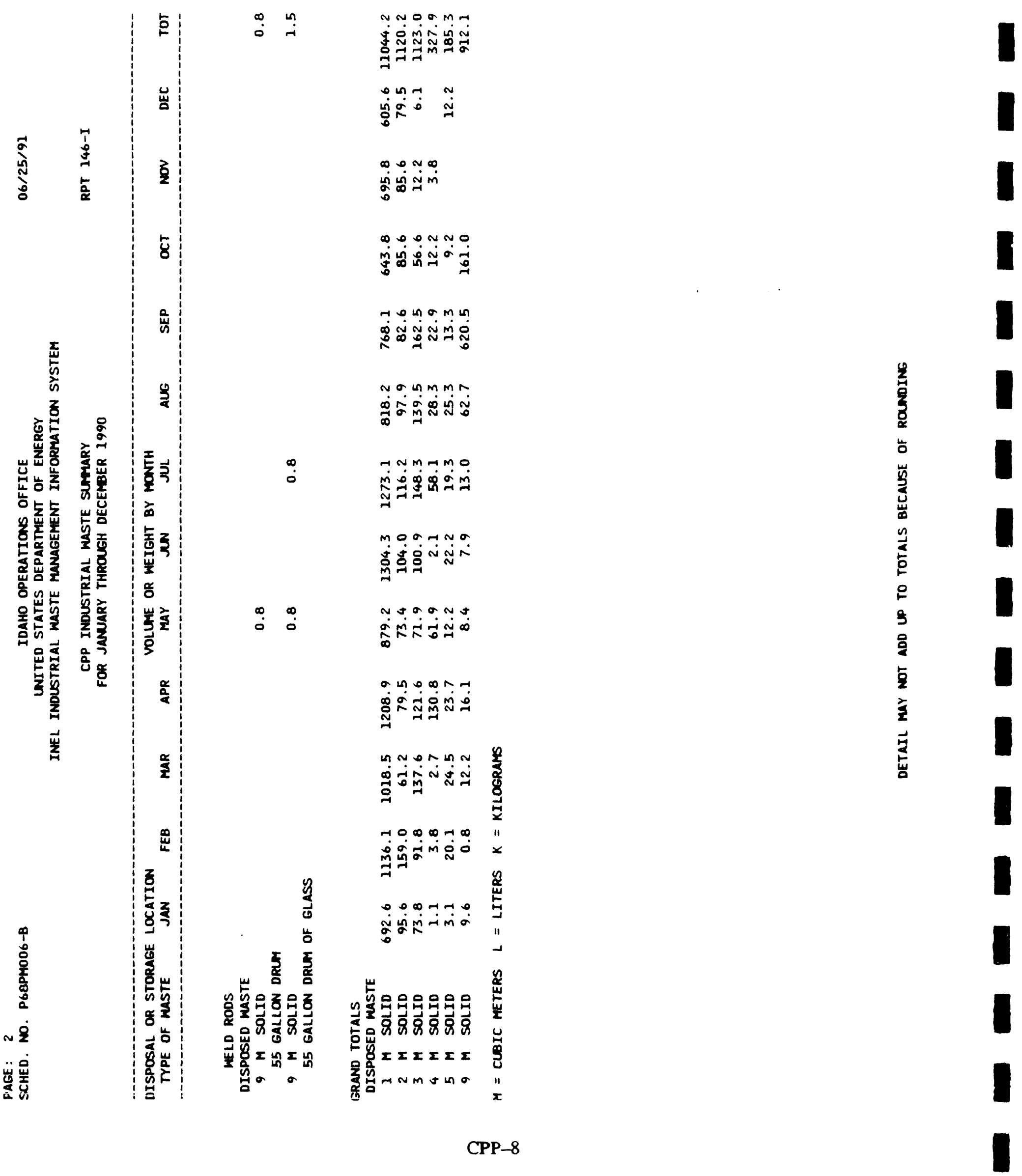
I
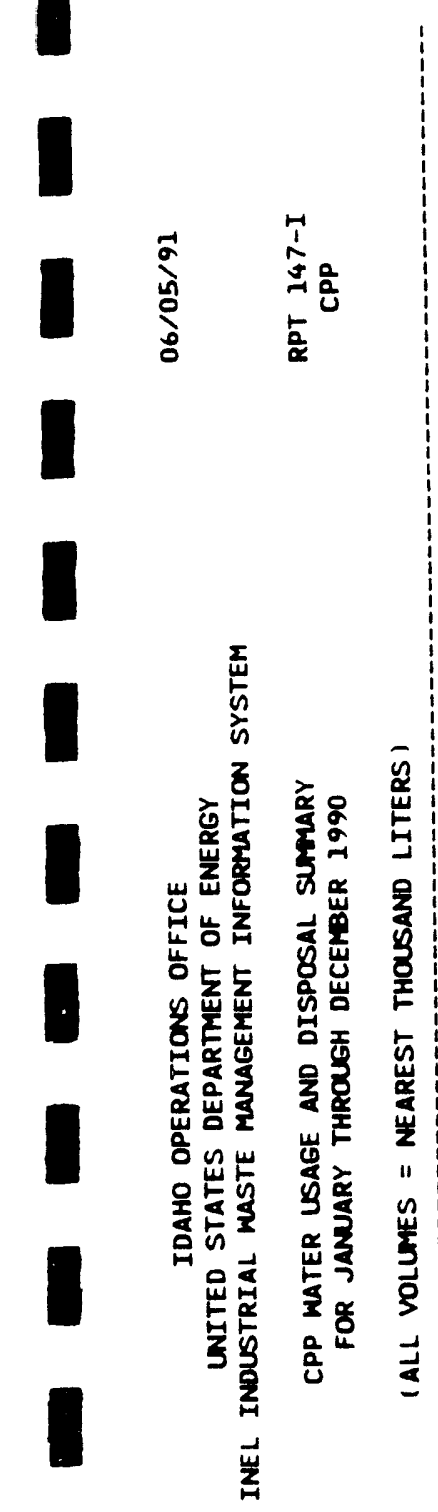

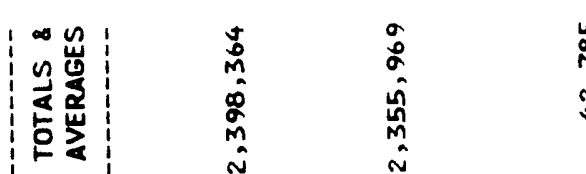

岀

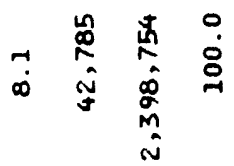

m

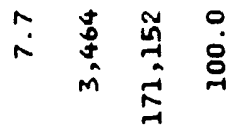

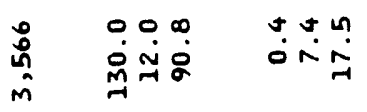

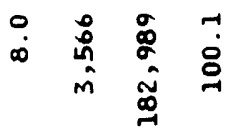

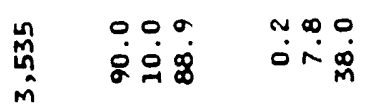

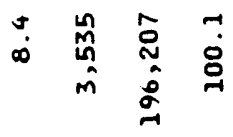

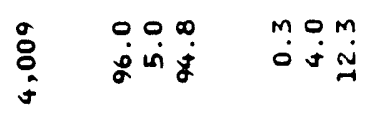

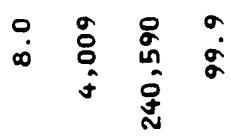

突

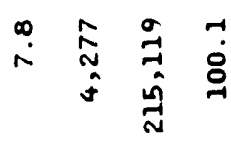

की

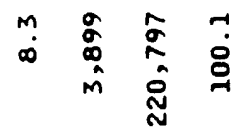

草

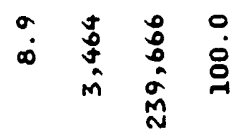

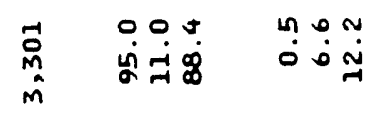

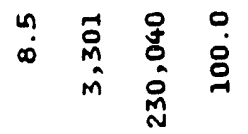

जे

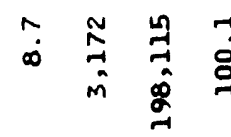

ஸे मूं

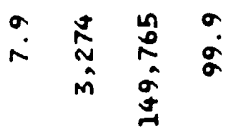

न

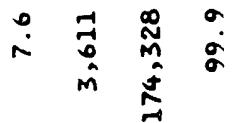

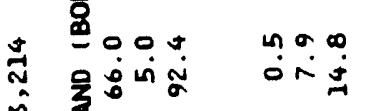

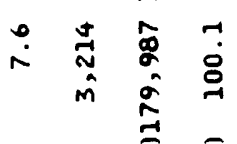

苞密 


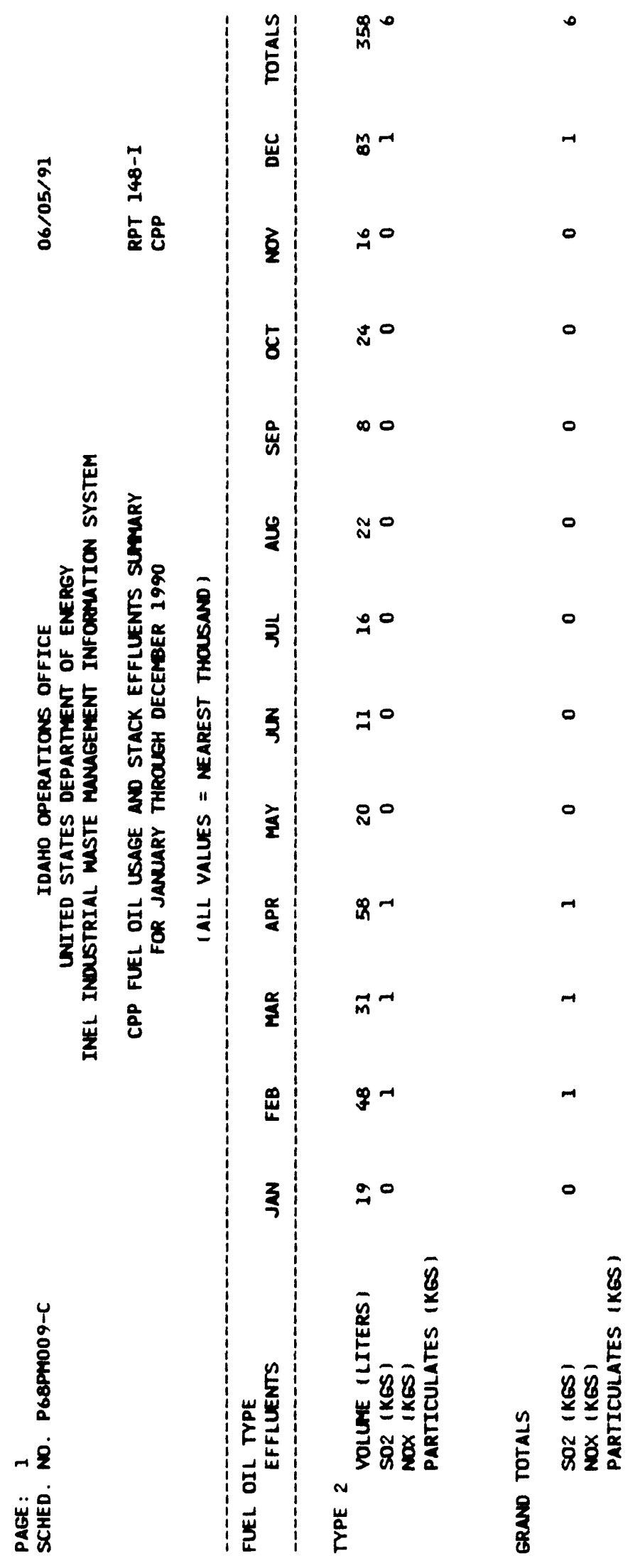

CPP-10 


\section{CONTAINMENT TEST FACILITY, 1990 DETAIL DATA}

Report 165 - CTF Liquid Disposed Substances Summary $\ldots \ldots \ldots \ldots \ldots$ CTF-3

Report 167 - CTF Water Usage and Disposal Summary . . . . . . . . CTF-6

Report 168 - CTF Fuel Oil Usage and Stack Effluents Summary . . . . . . . CTF-7 


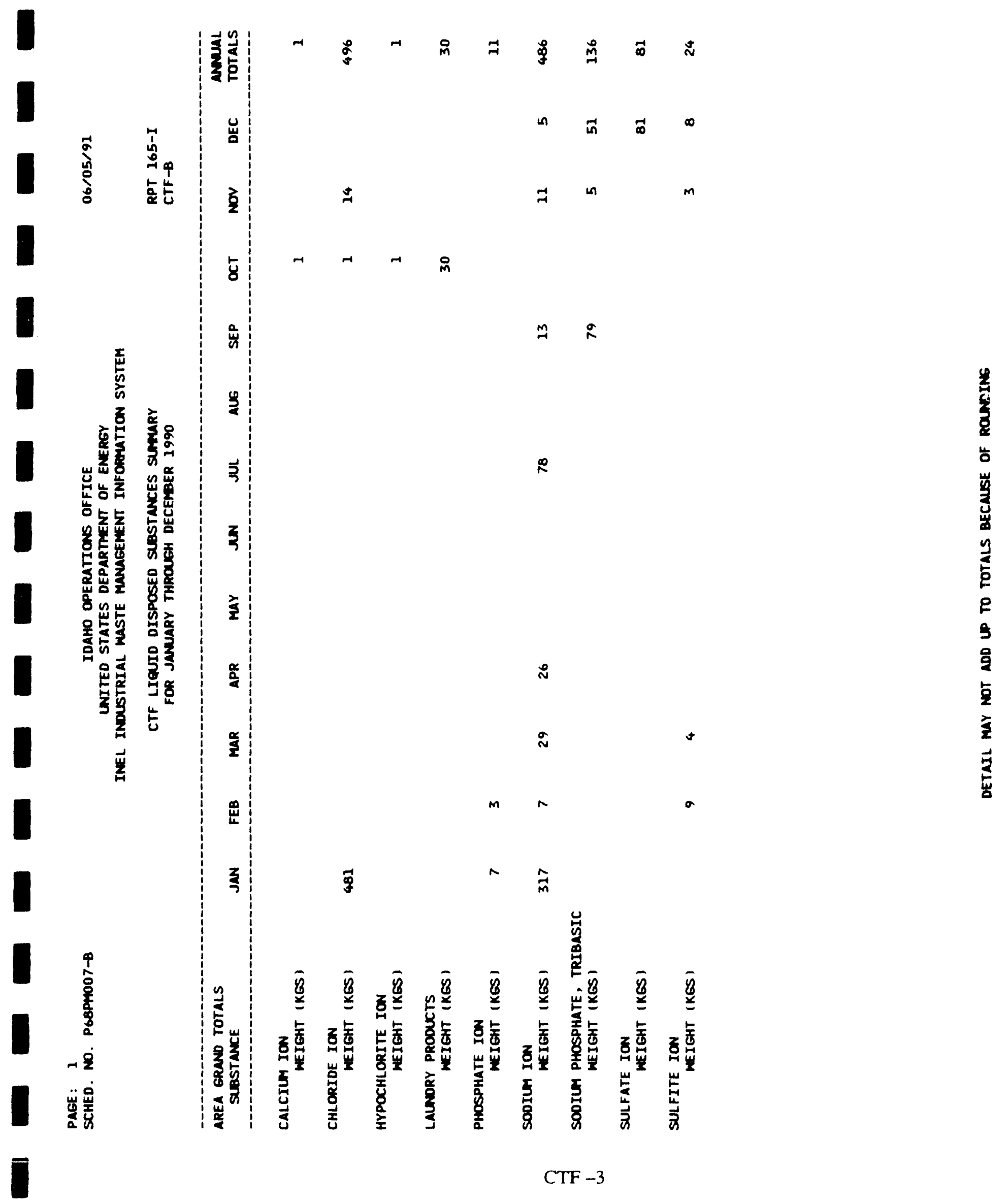




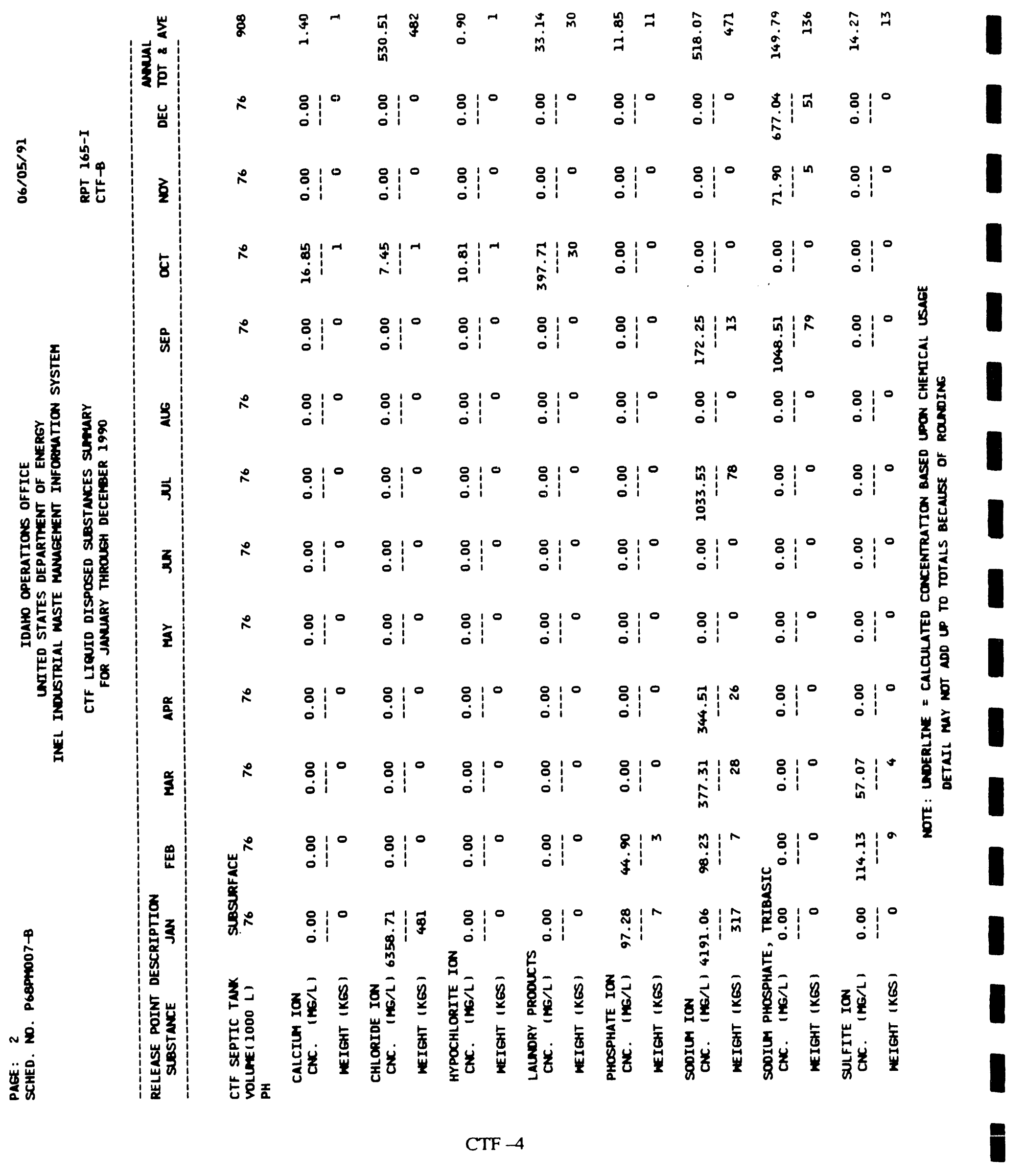


I

I

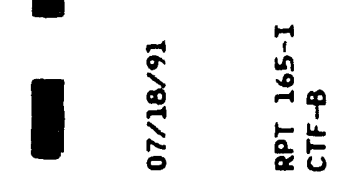

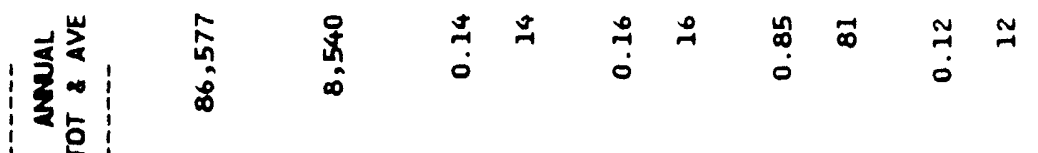

I

I

1

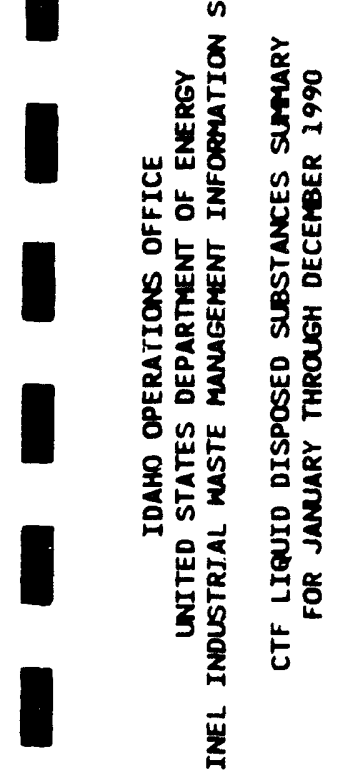

I

I

I

$\sim \dot{z}$

譬案
落

3)

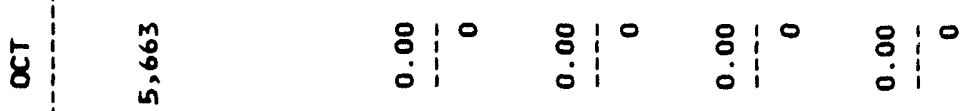

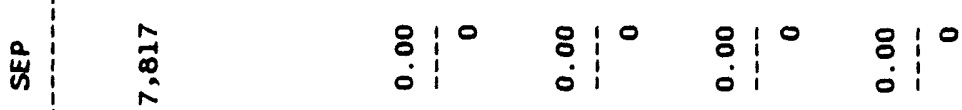

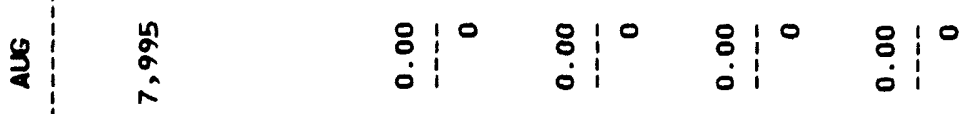

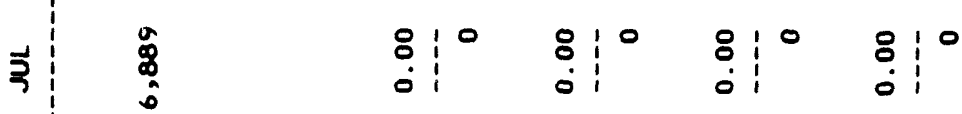

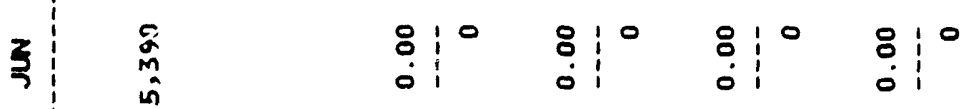

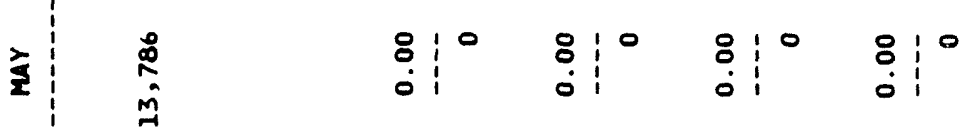

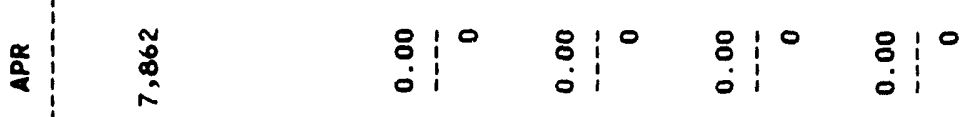

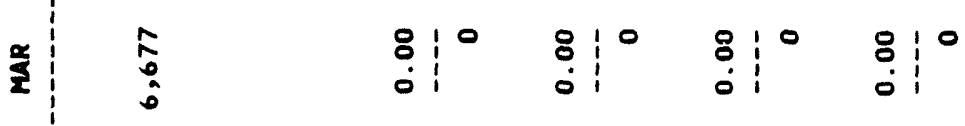

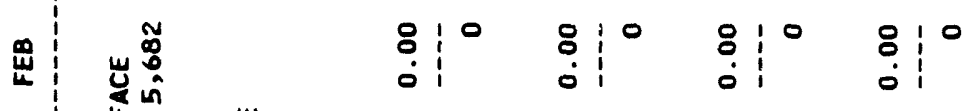

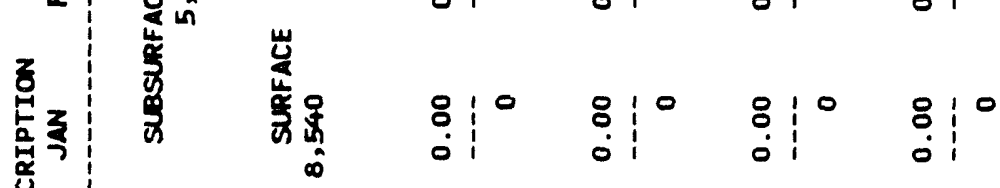

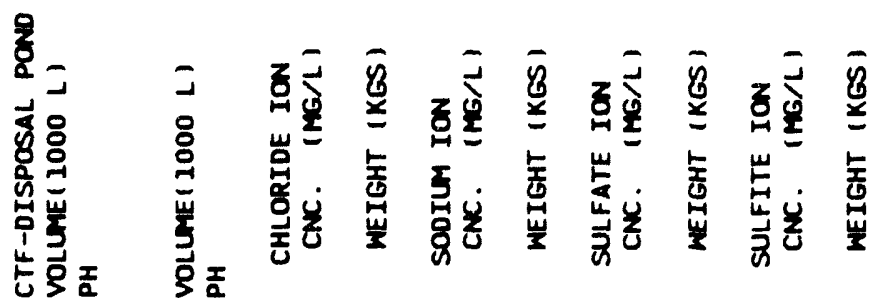




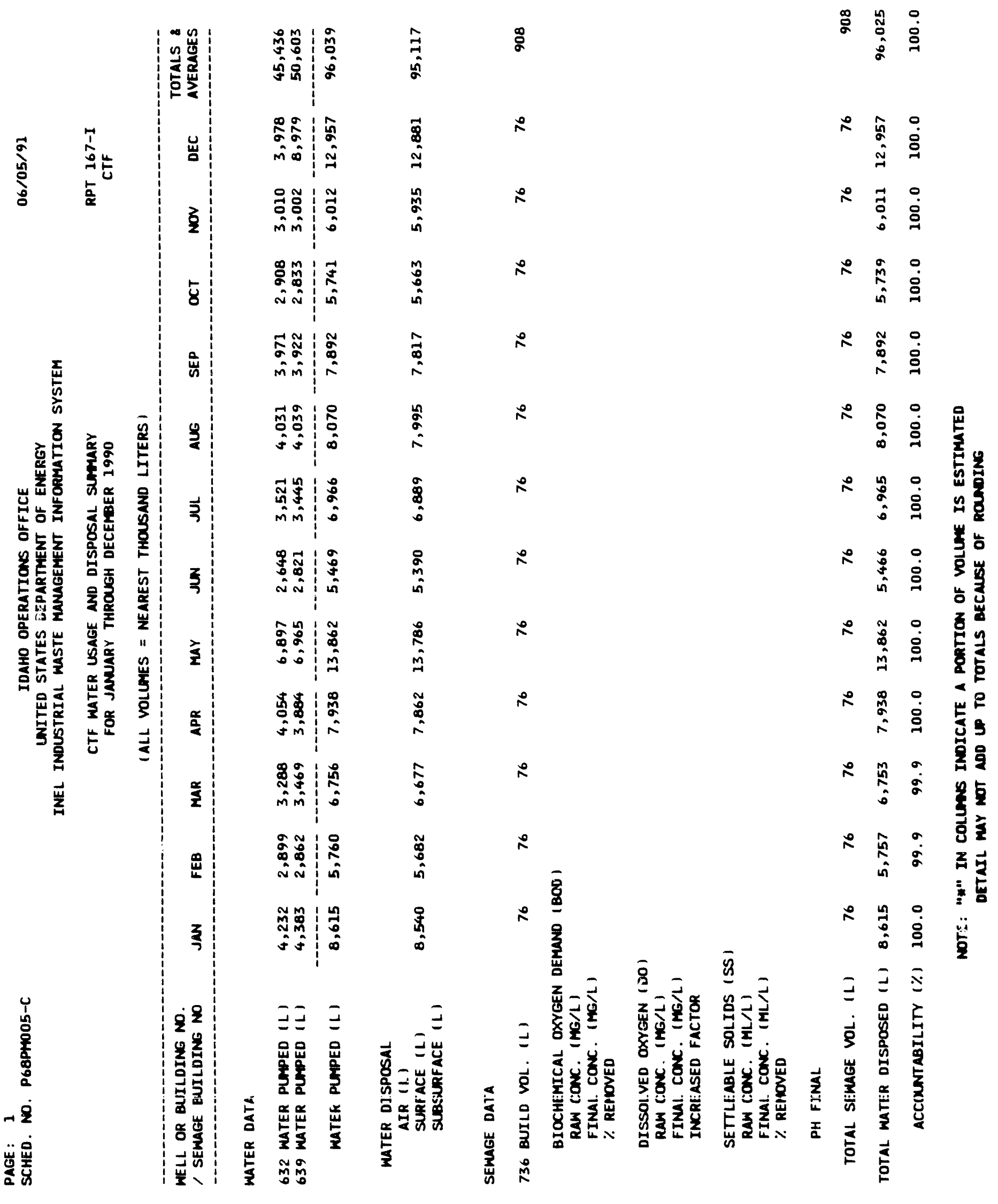




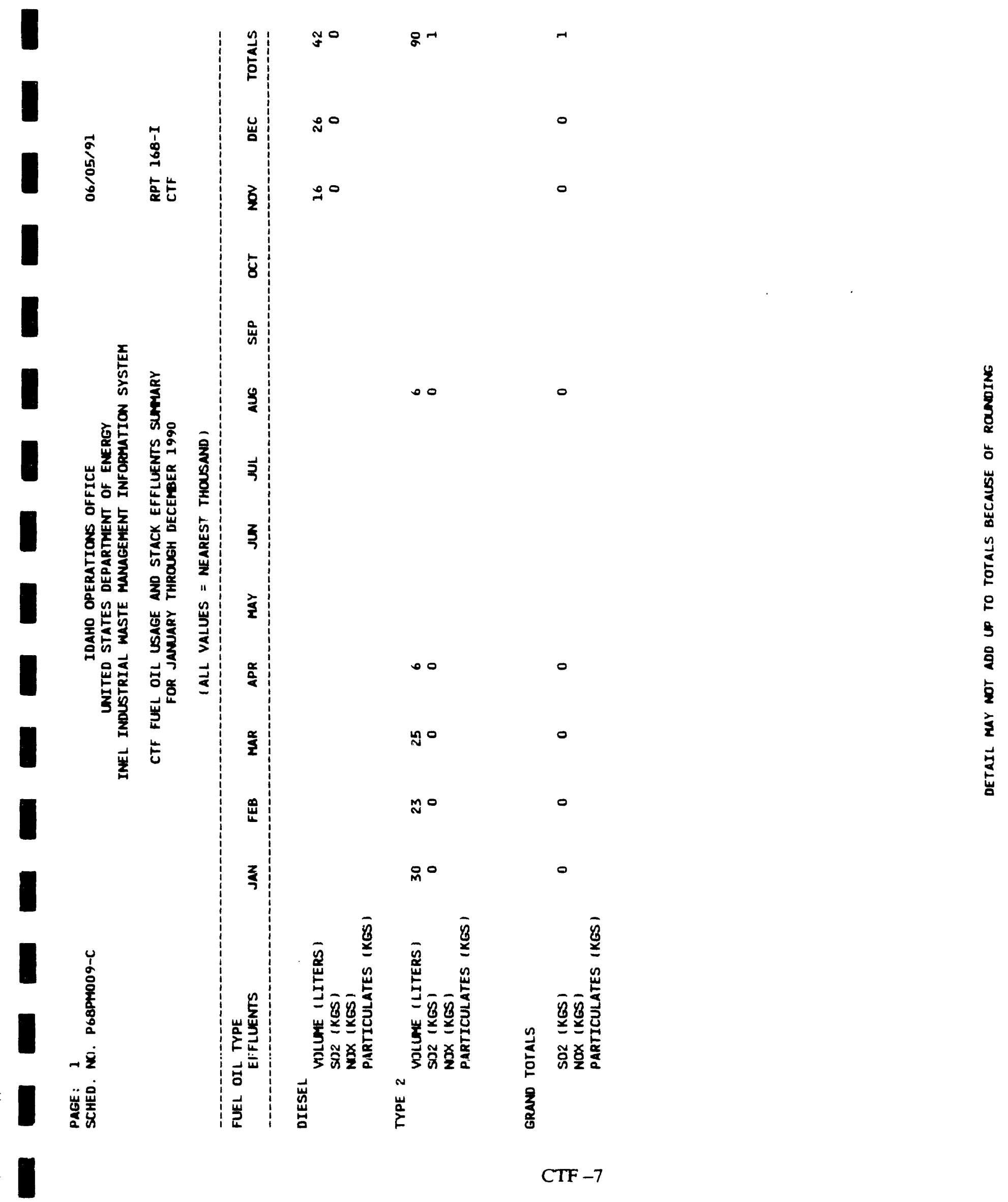


MAGNETOHYDRODYNAMICS COMPONENT DEVELOPMENT, 1990 DETAIL DATA

Report $176-$ MHD Industrial Waste Summary $\ldots \ldots \ldots \ldots \ldots \ldots \ldots$ MHD-3

Report 177 - MHD Water Usage and Disposal Summary $\ldots \ldots \ldots \ldots \ldots$ MHD-4

Report 178 - MHD Fuel Oil Usage and Stack Effiuents Summary . . . . . . . . MHD-5 
I

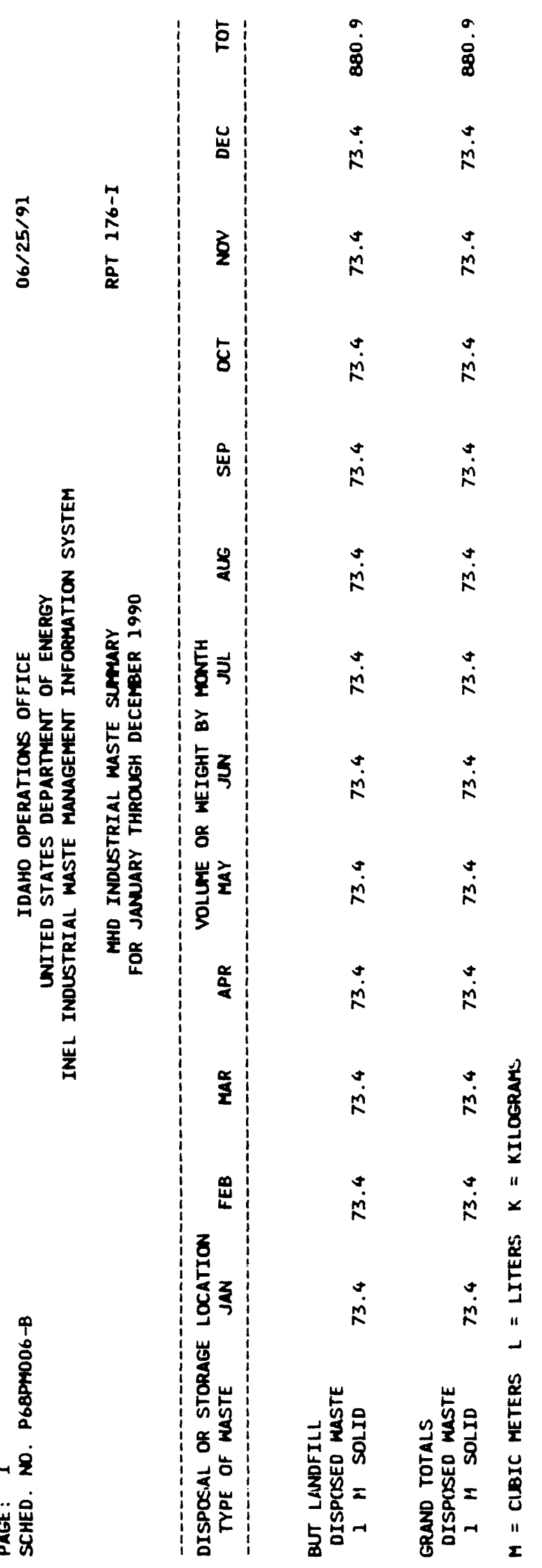

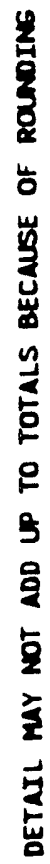




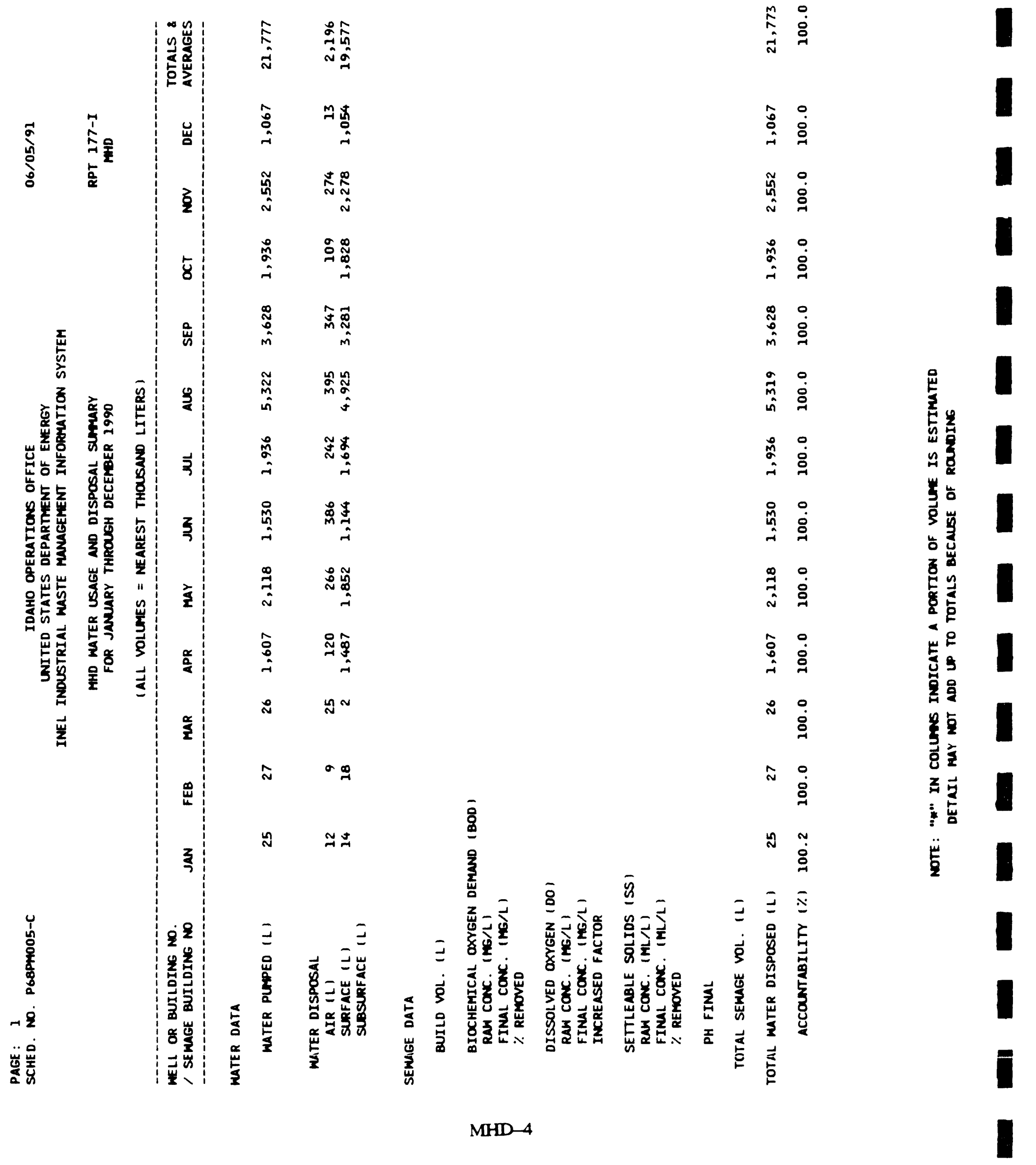




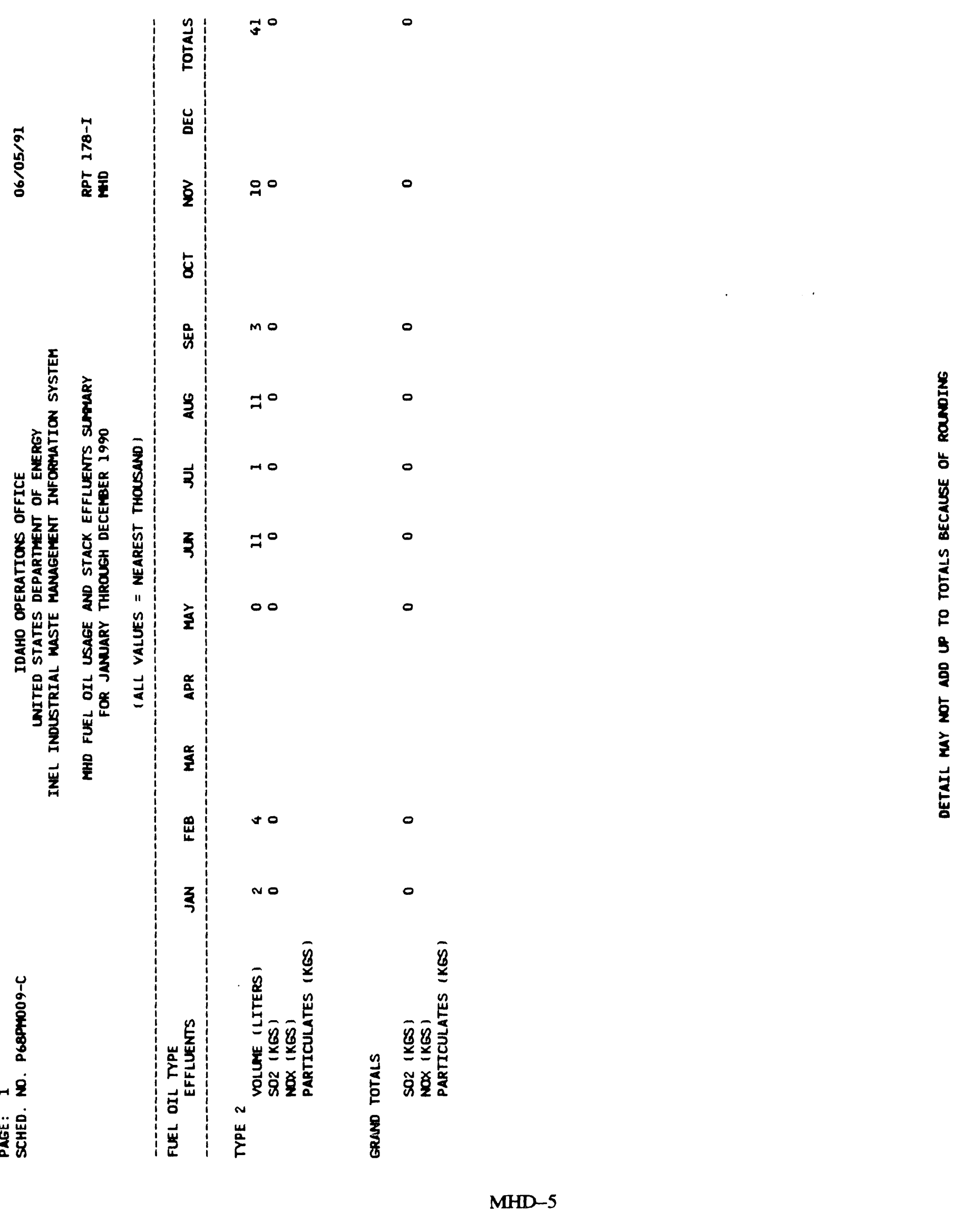


NAVAL REACTORS FACILITY, 1990 DETAIL DATA

Report 184 - NRF Airborne Disposed Substances Summary .......... NRF-3

Report 185 - NRF Liquid Disposed Substances Summary ............ NRF-4

Report 186 - NRF Industrial Waste Summary $\ldots \ldots \ldots \ldots \ldots \ldots \ldots \ldots \ldots \ldots \ldots$ NRF-7

Report 187 - NRF Water Usage and Disposal Summary $\ldots \ldots \ldots \ldots \ldots \ldots$ NRF-8

Report 188 - NRF Fuel Oil Usage and Stack Effluents Summary .......... NRF-9 


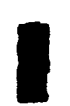

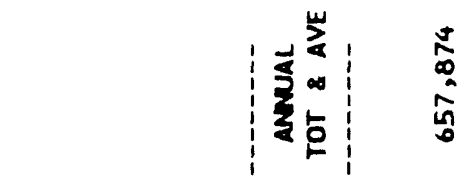

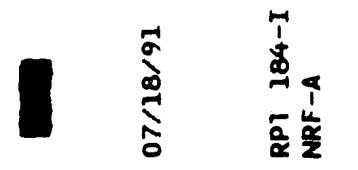

㟧

I

I

I

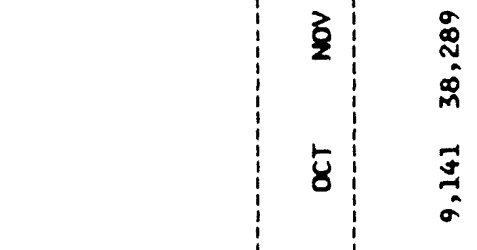

I

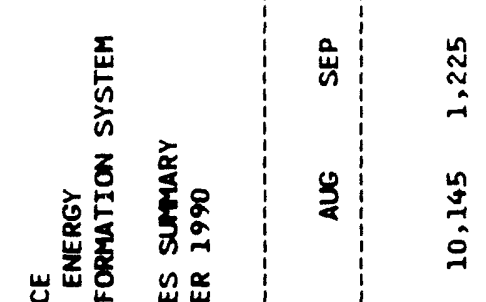

I

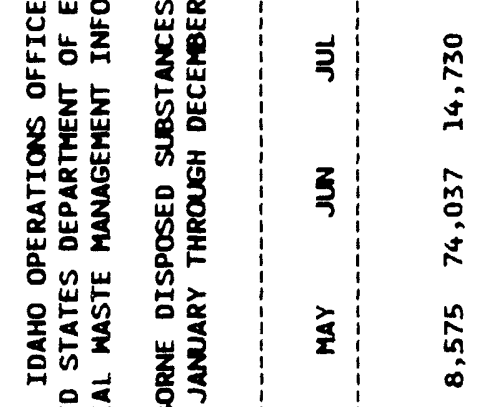

胥

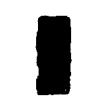

sa

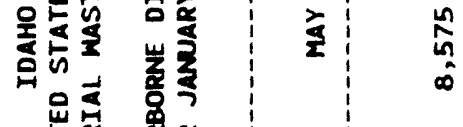

0
0
0
0
0
0
0
0
0
0
0

I

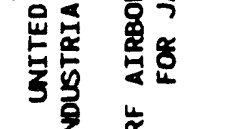

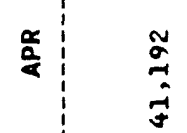

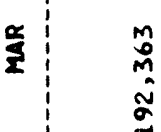

崫

I

I

京

$-\dot{q}$

警案

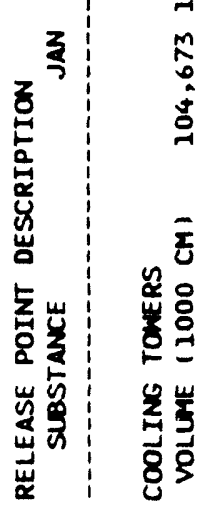




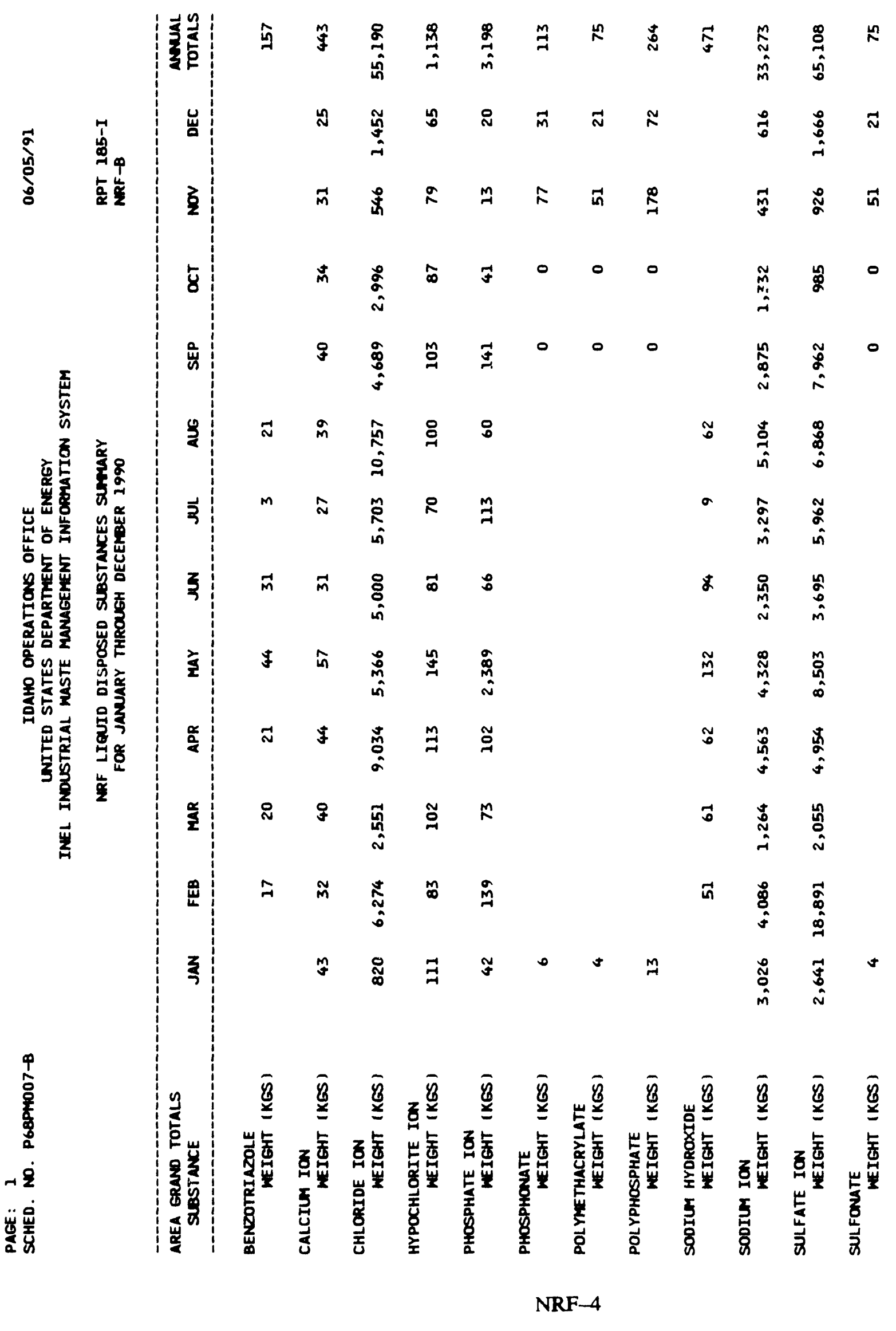

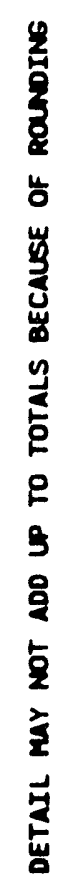


1

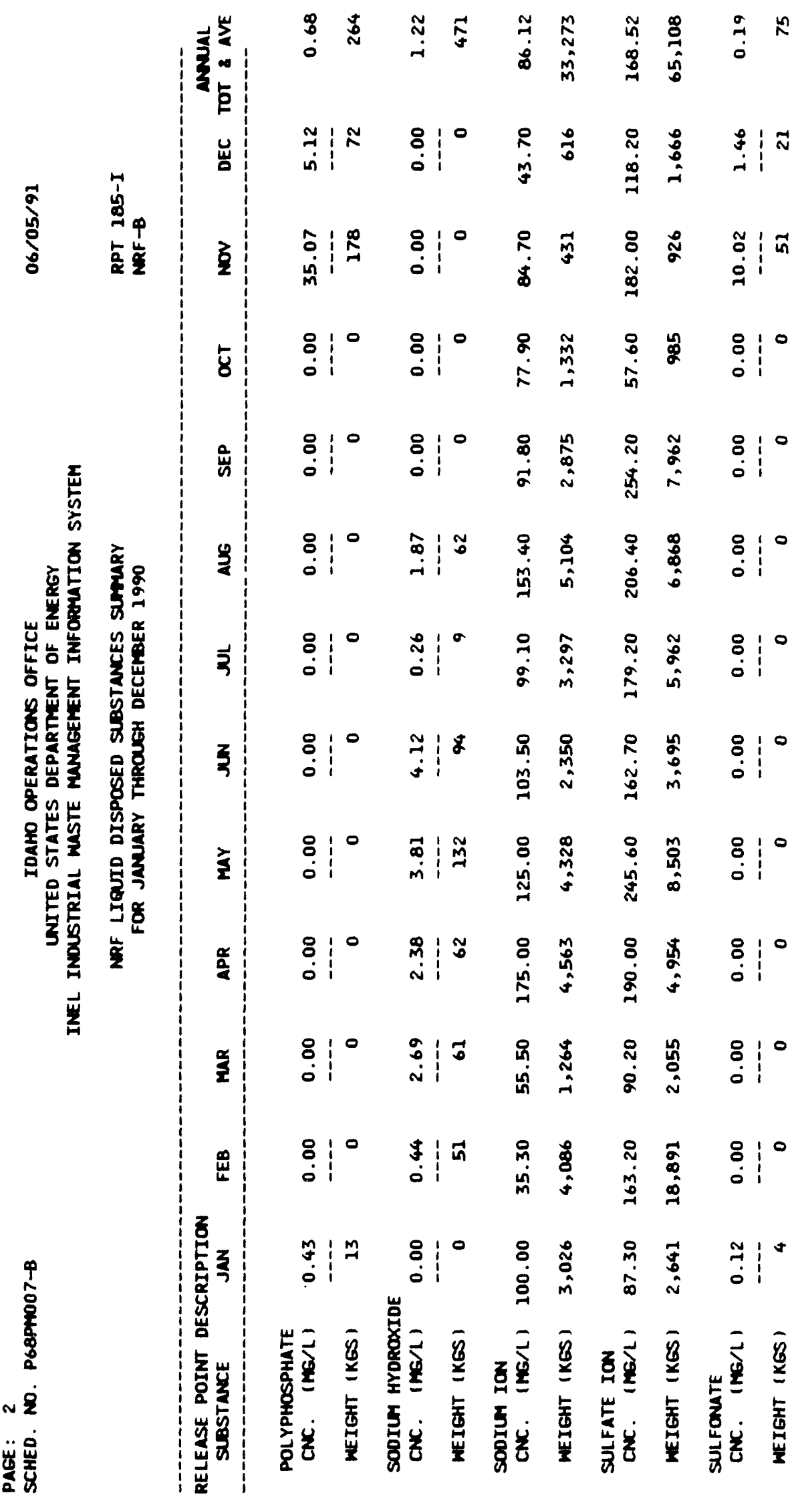




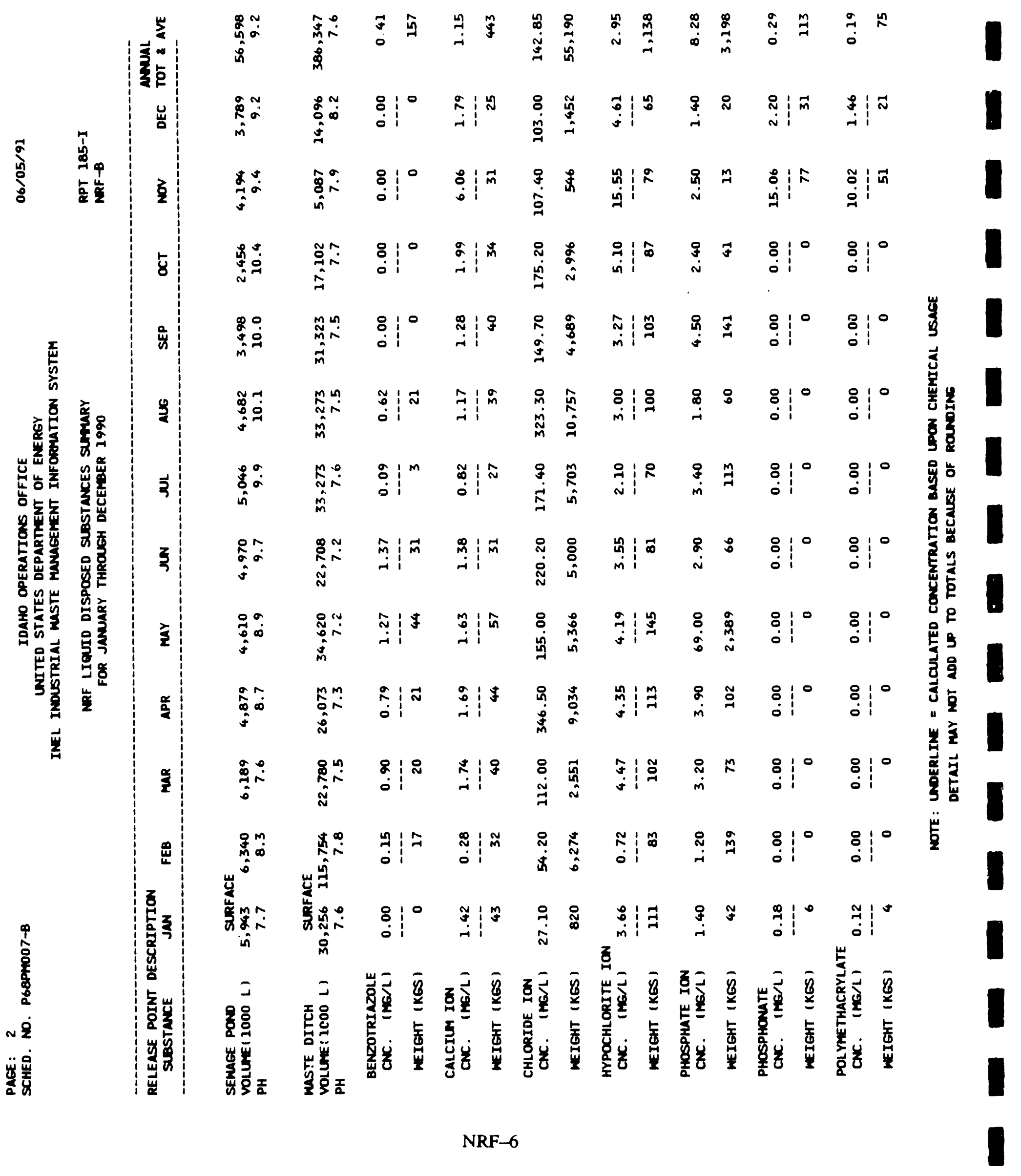




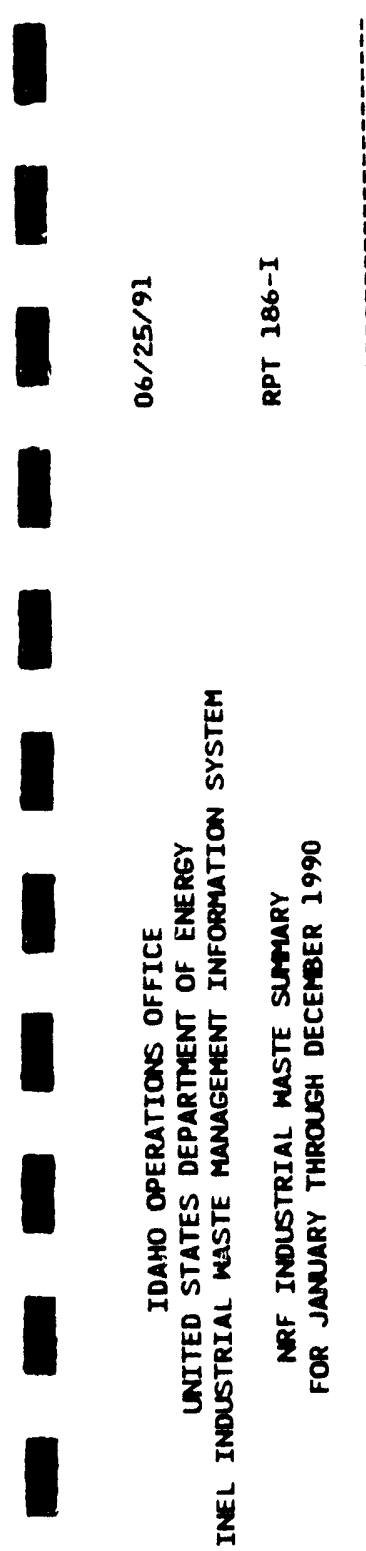

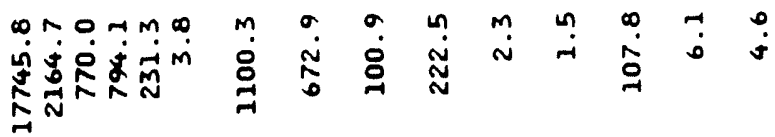

$\infty \wedge 0.1 m \infty$

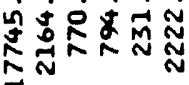

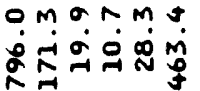

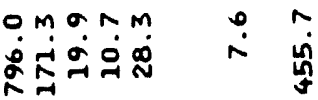

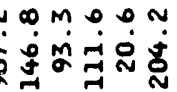

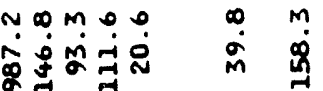

$\overrightarrow{0}$

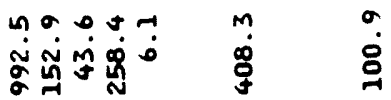

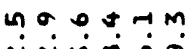

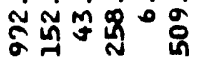

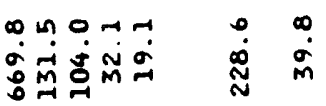

$\dot{j}$

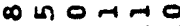

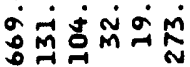

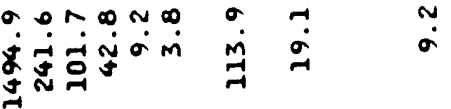

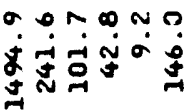

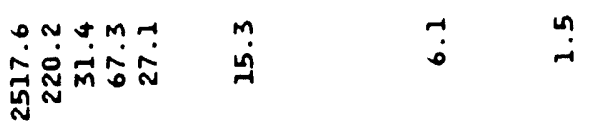

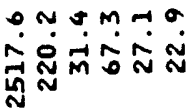

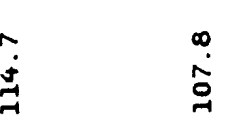

on noom

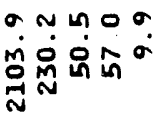

$\stackrel{4}{4}$

อับำ

응

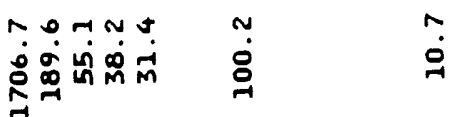

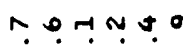

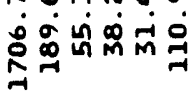

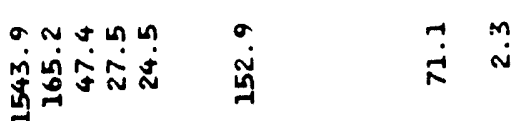

a n U Unm

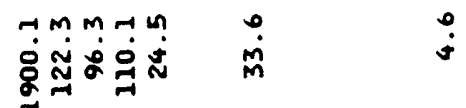

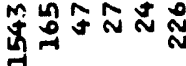

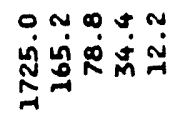

$\dot{0}$

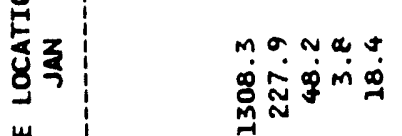

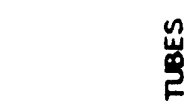

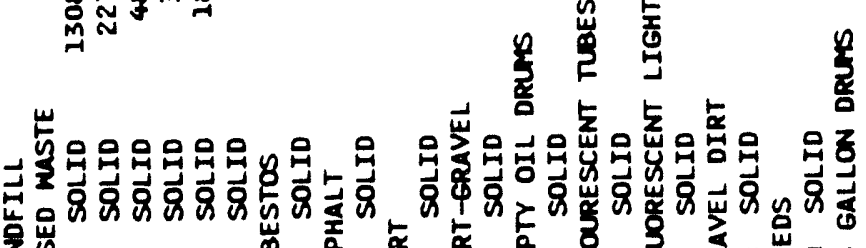

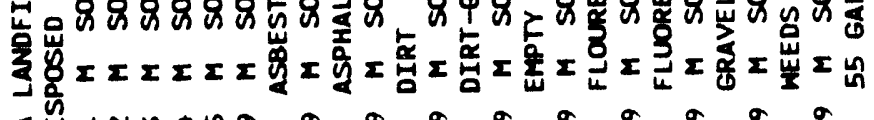

O

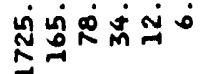

man

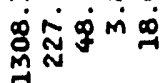

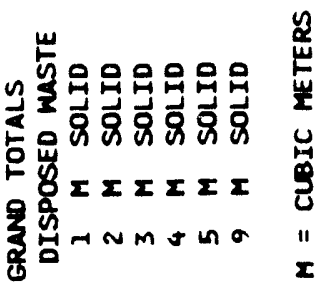




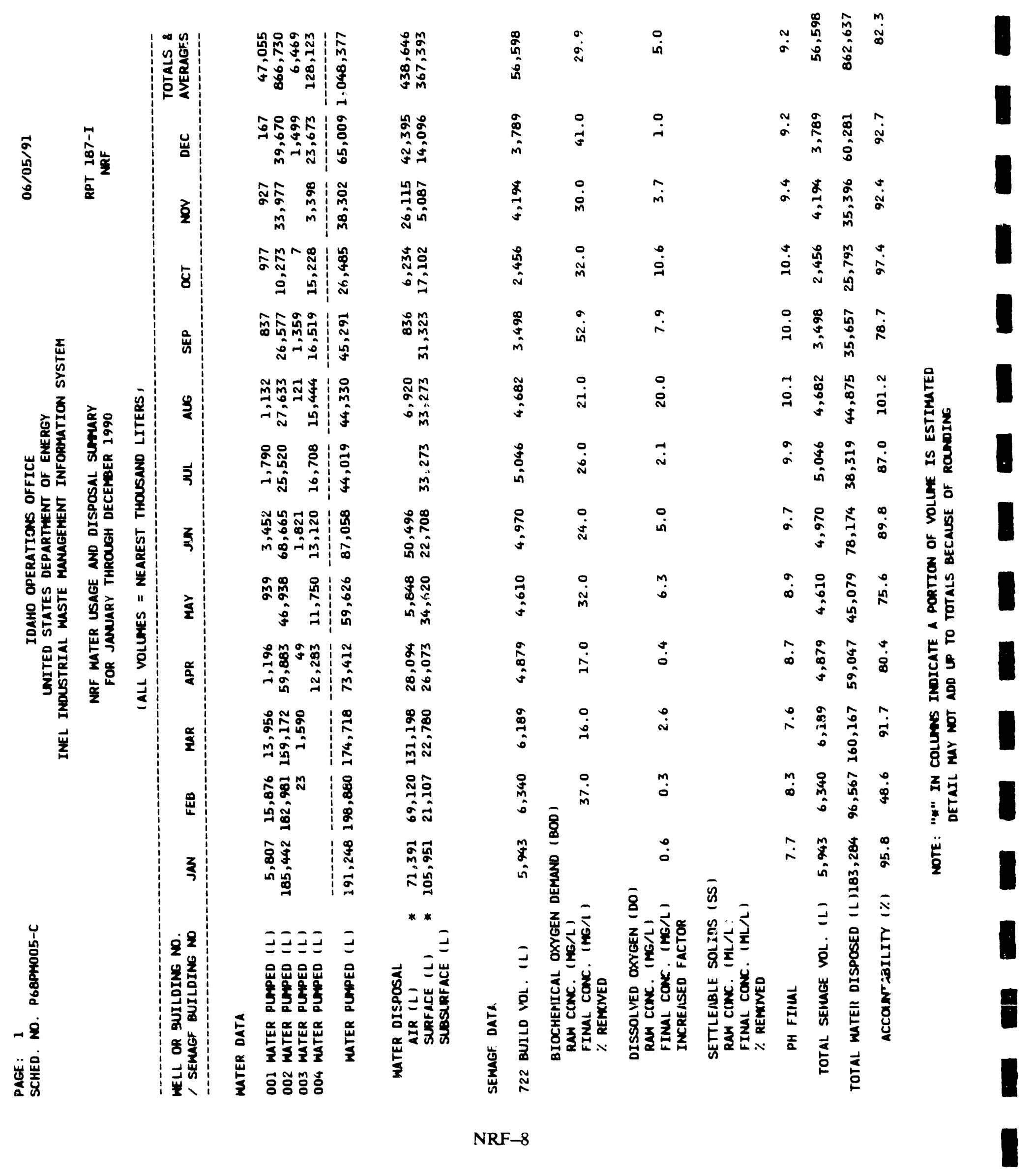




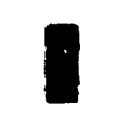

I!

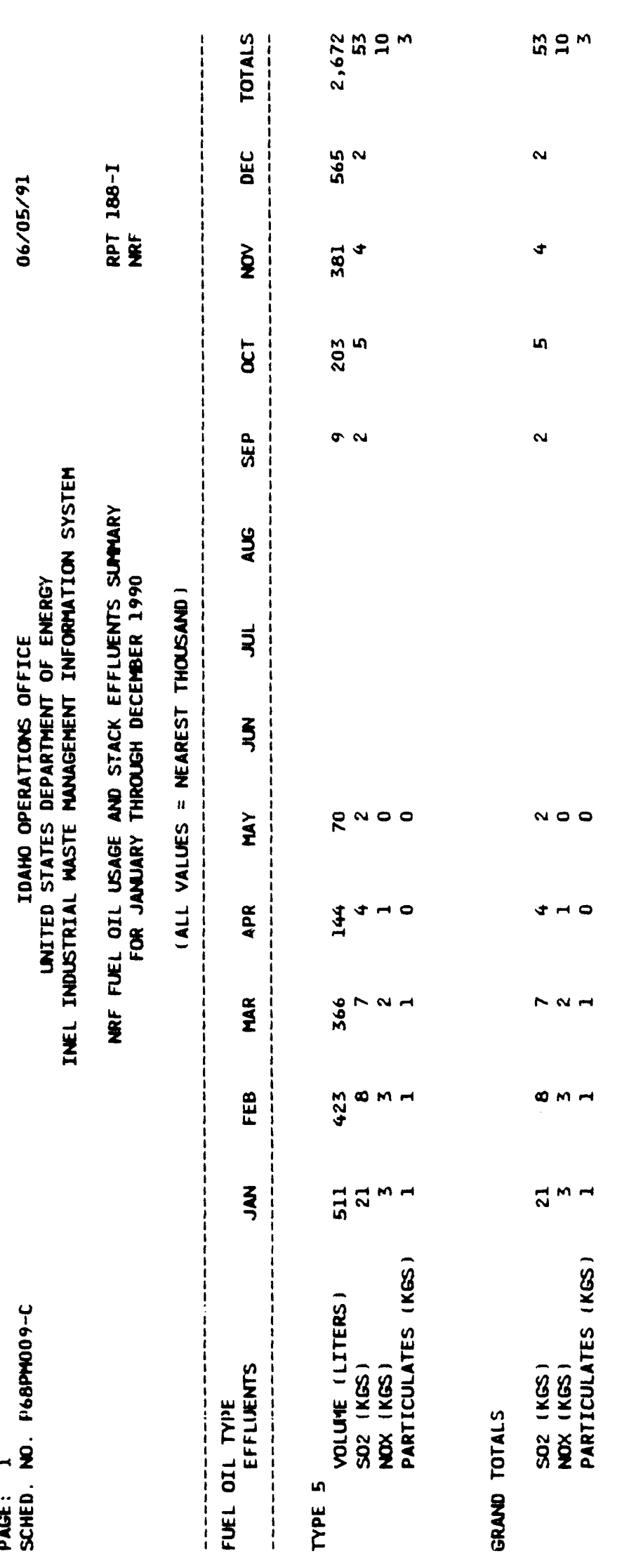


POWER BURST FACILITY, 1990 DETAIL DATA

Report 195 - PBF Liquid Disposed Substances Summary ............ PBF-3

Report 196 -. PBF Industrial Waste Summary $\ldots \ldots \ldots \ldots \ldots \ldots \ldots \ldots$ PBF-6

Report 197 - PBF Water Usage and Disposal Summary . . . . . . . . . PBF-7

Report 198 - PBF Fuel Oil Usage and Stack Effluents Summan ${ }^{\circ} . \ldots \ldots \ldots \ldots$ PBF-9 


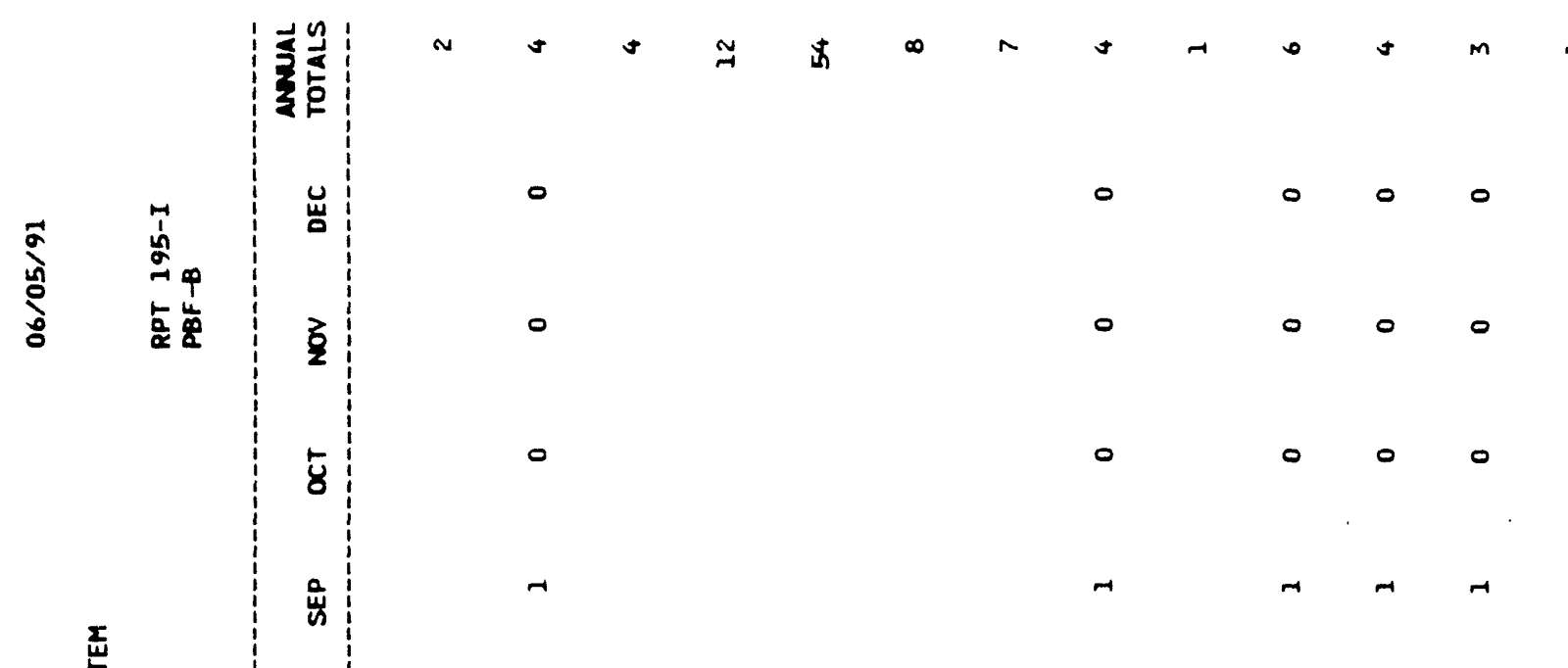

I

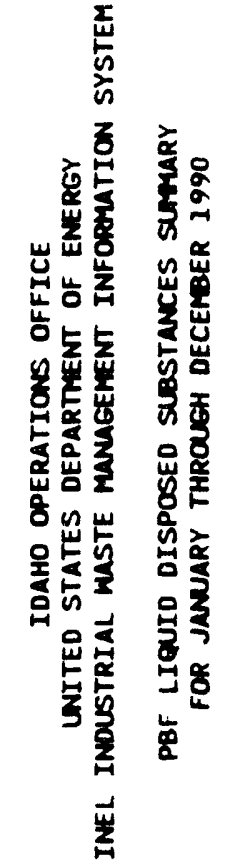

3

ง

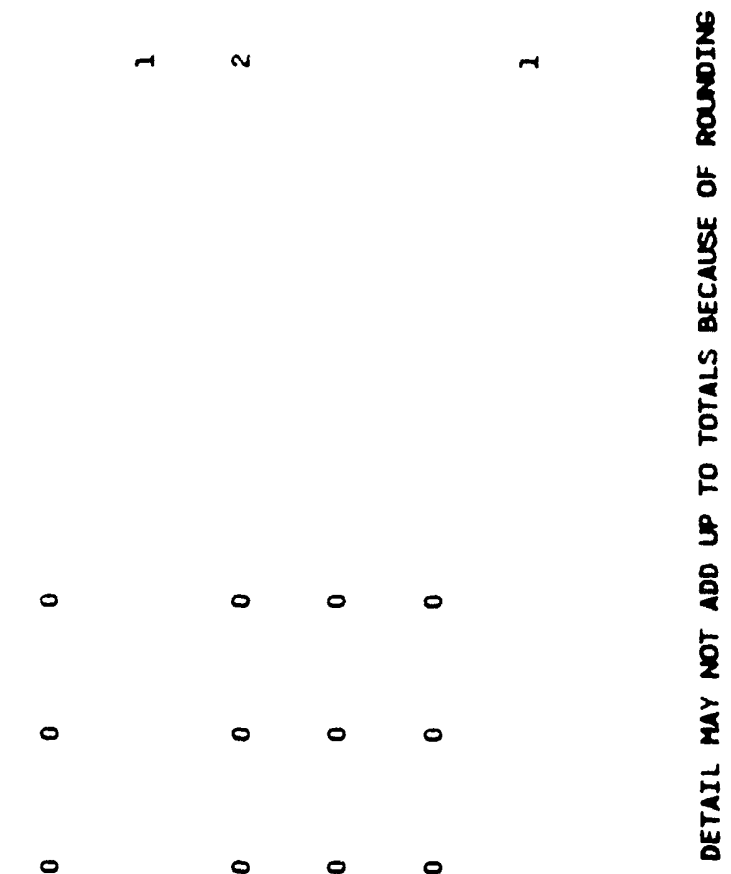

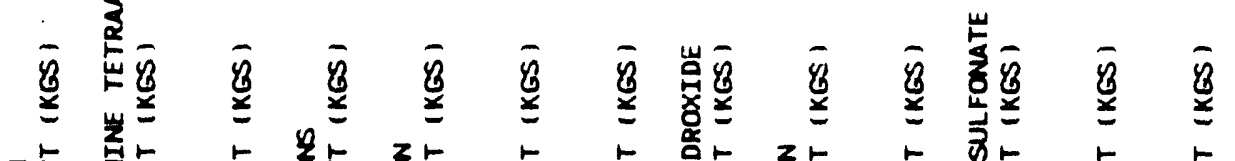




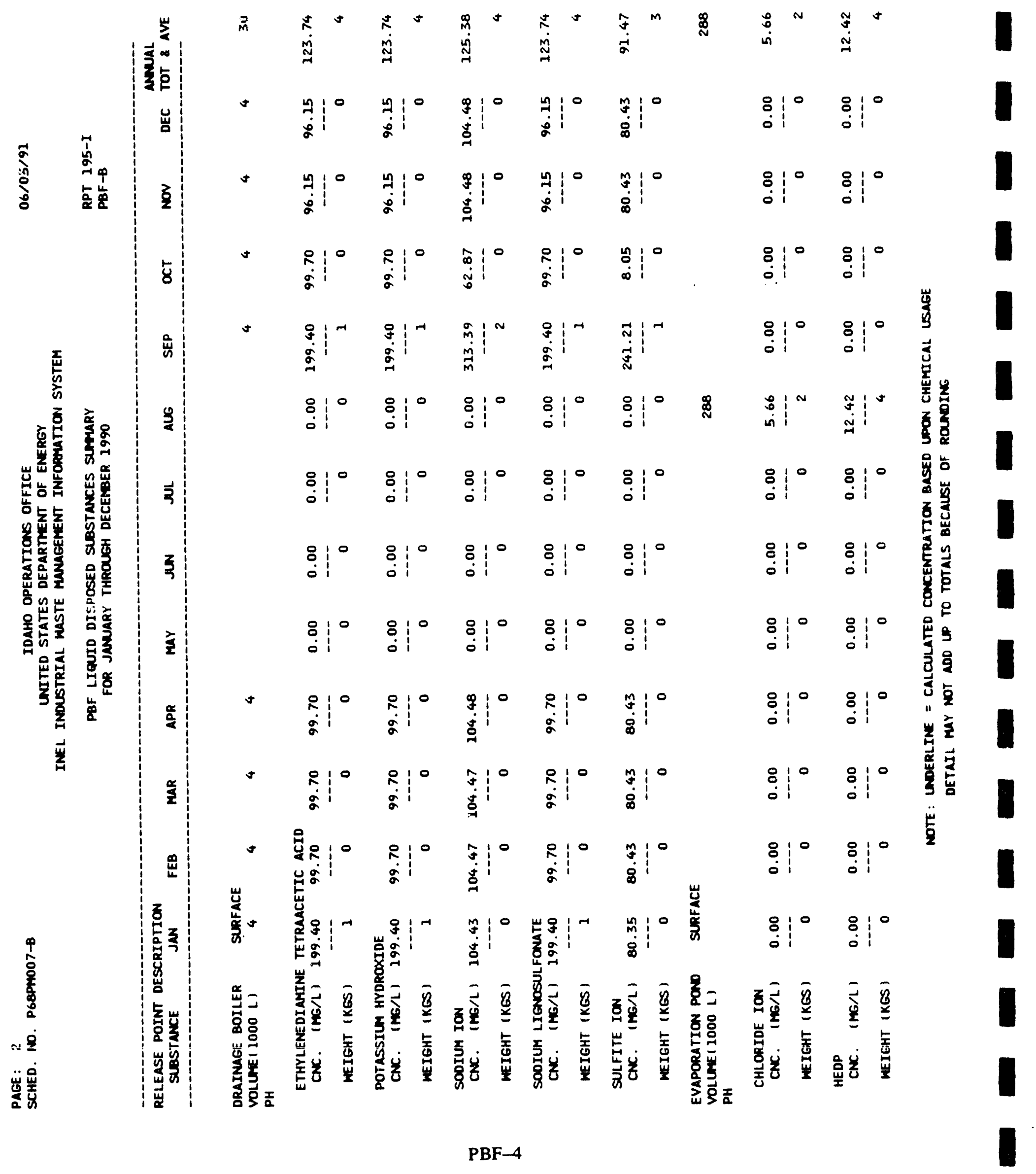


I

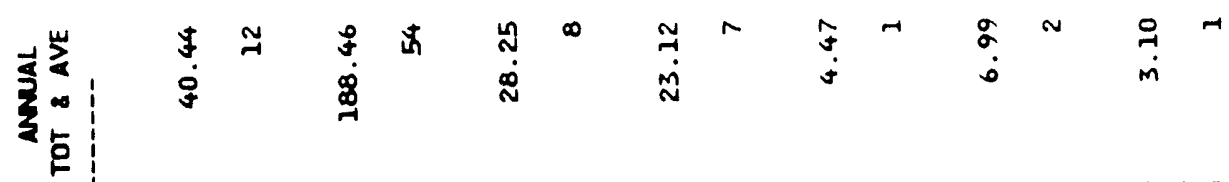

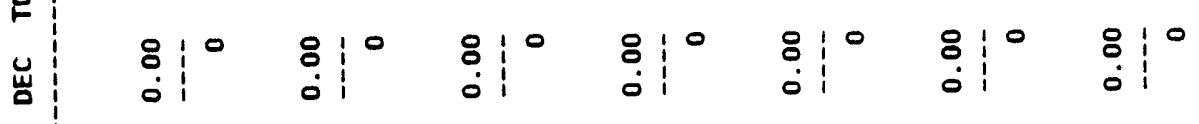

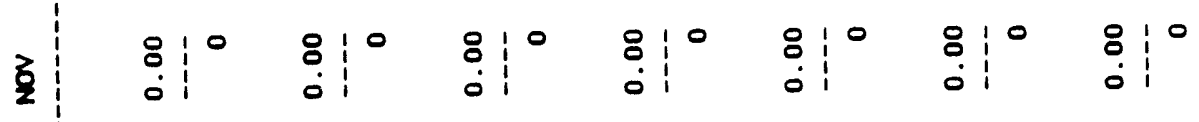

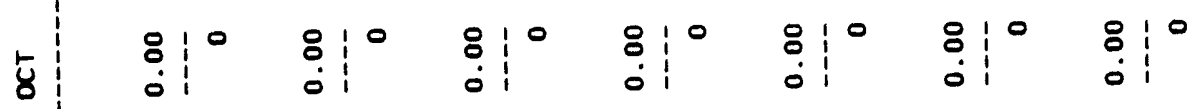

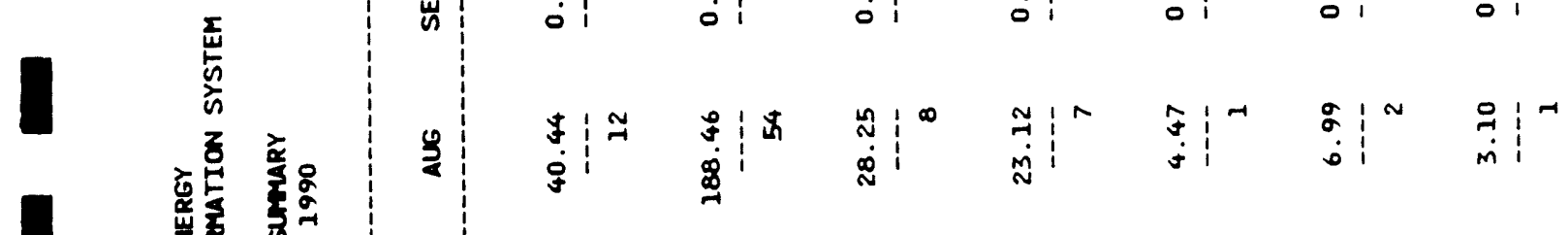

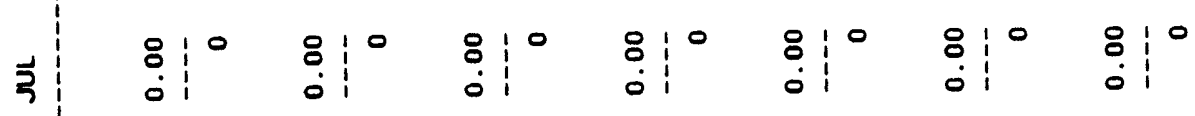

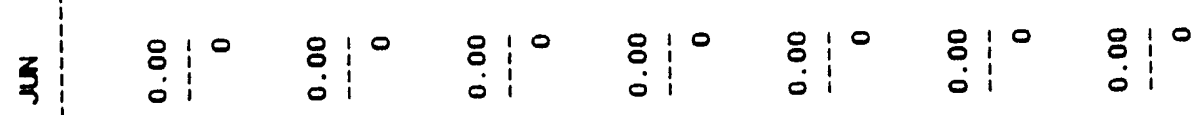

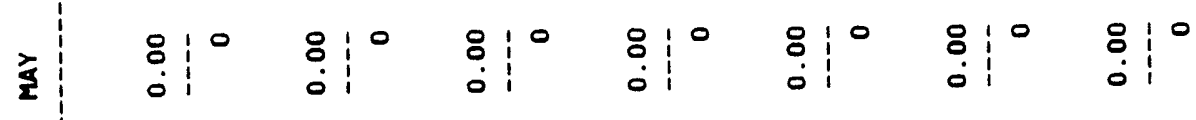

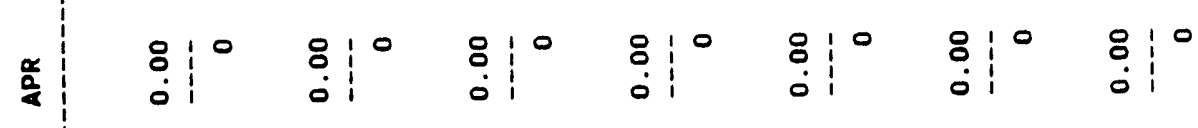

$\overrightarrow{\mathrm{m}}$

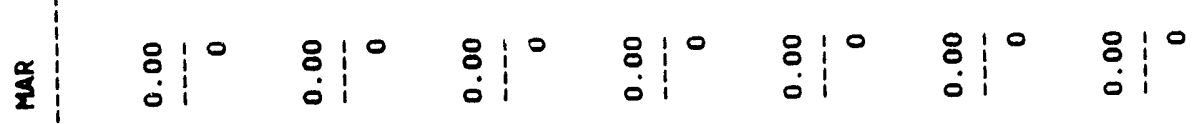

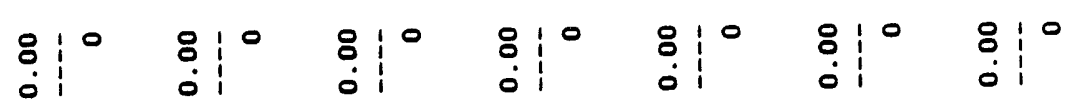

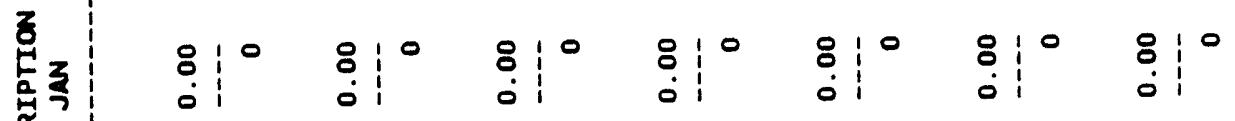

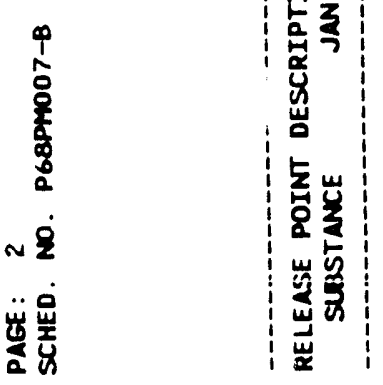

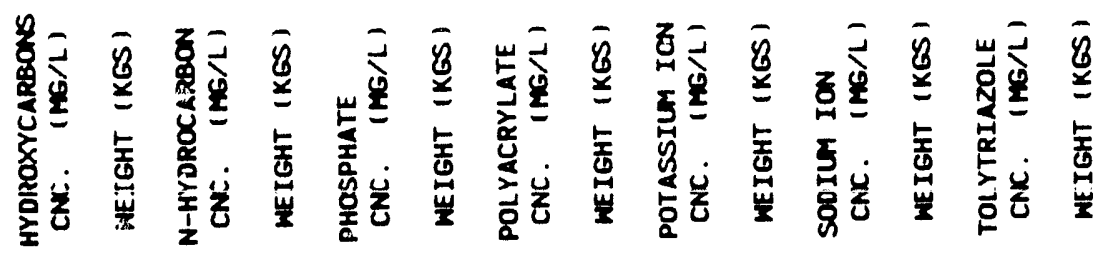




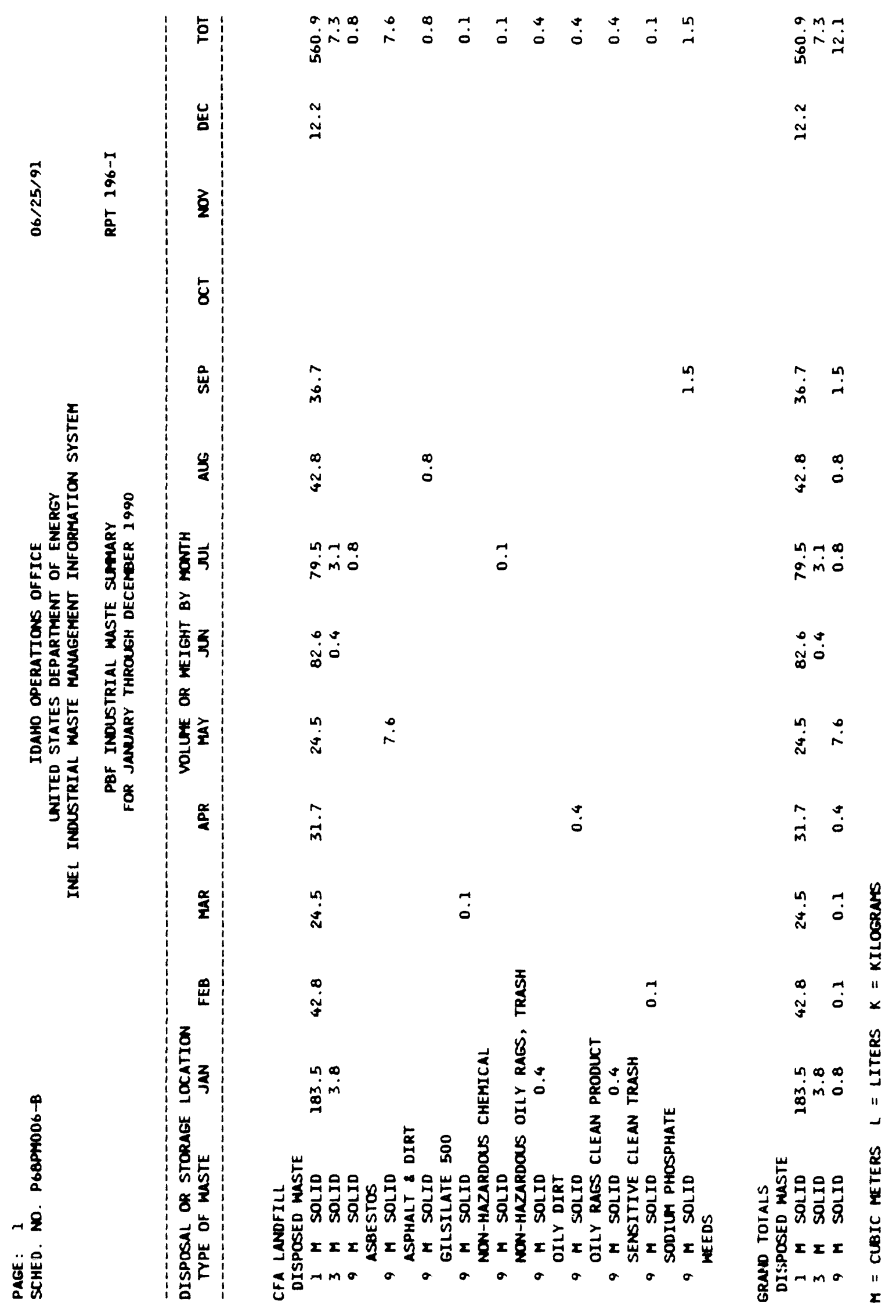

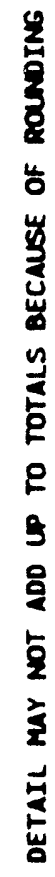




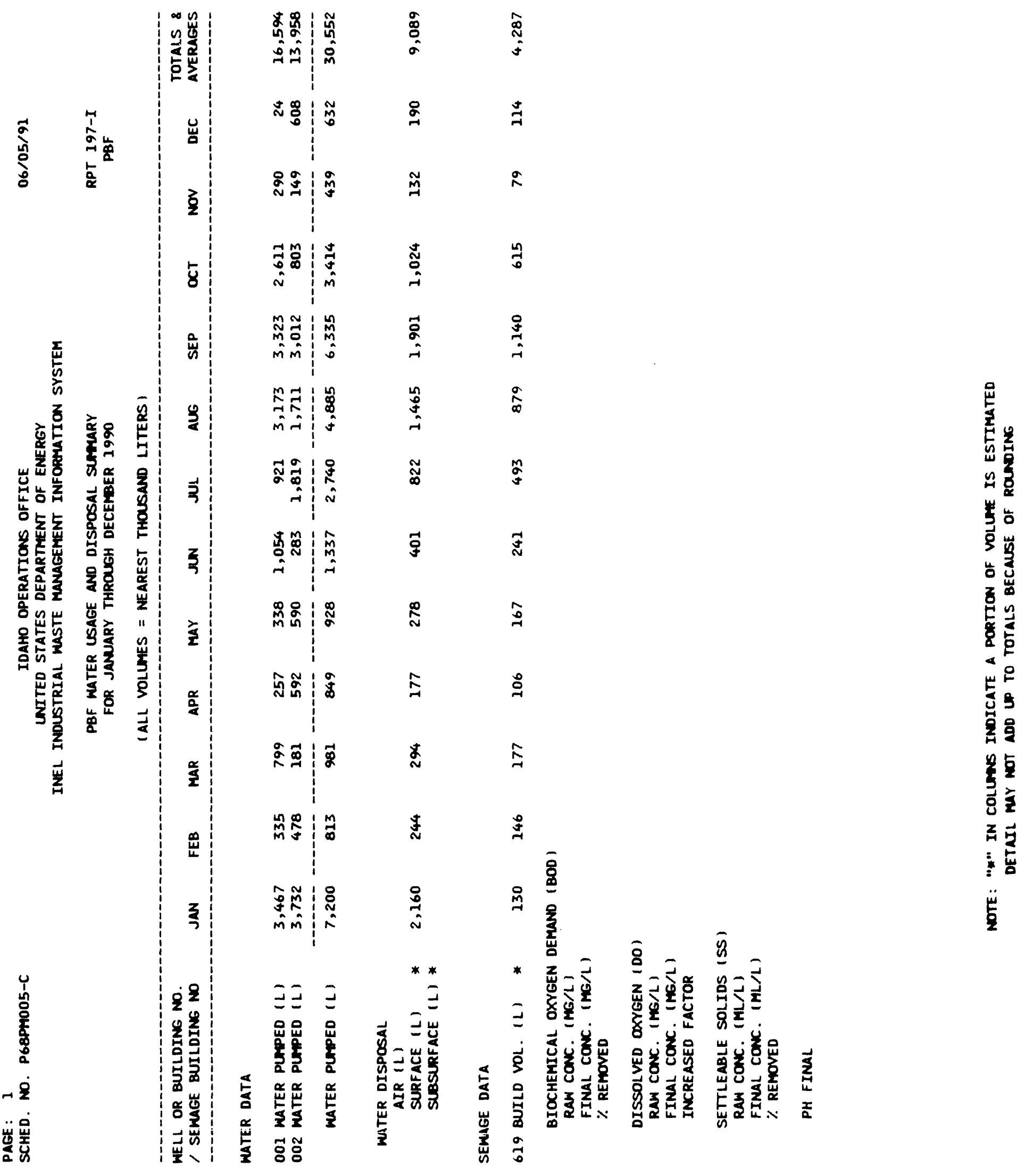



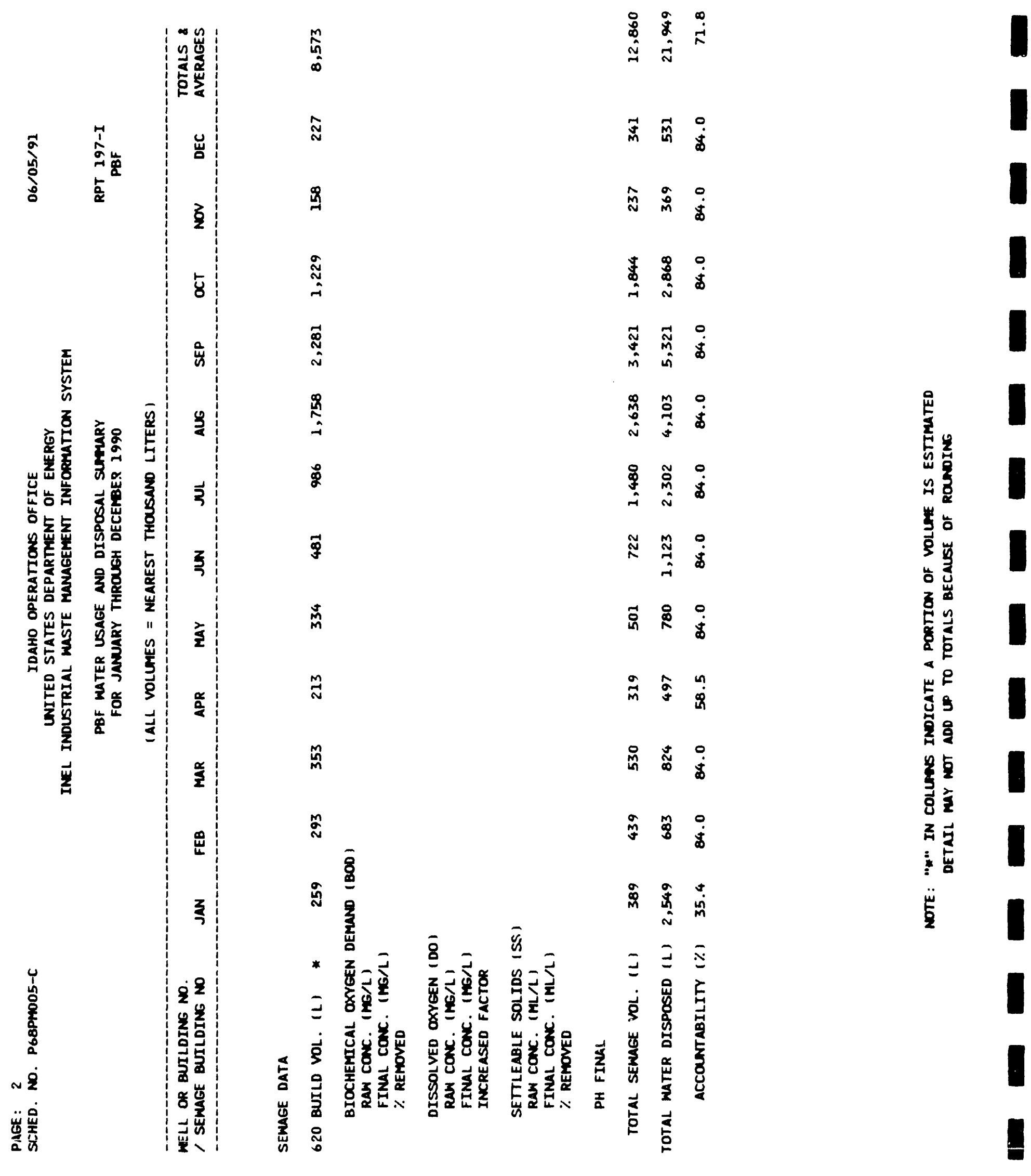

PBF-8 
I

I

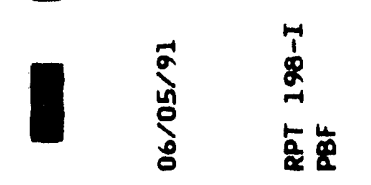

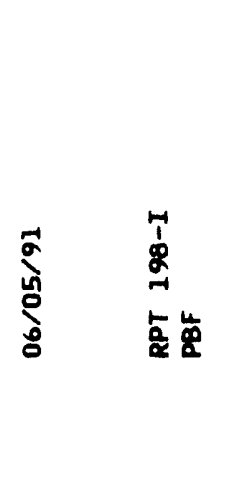

I

I
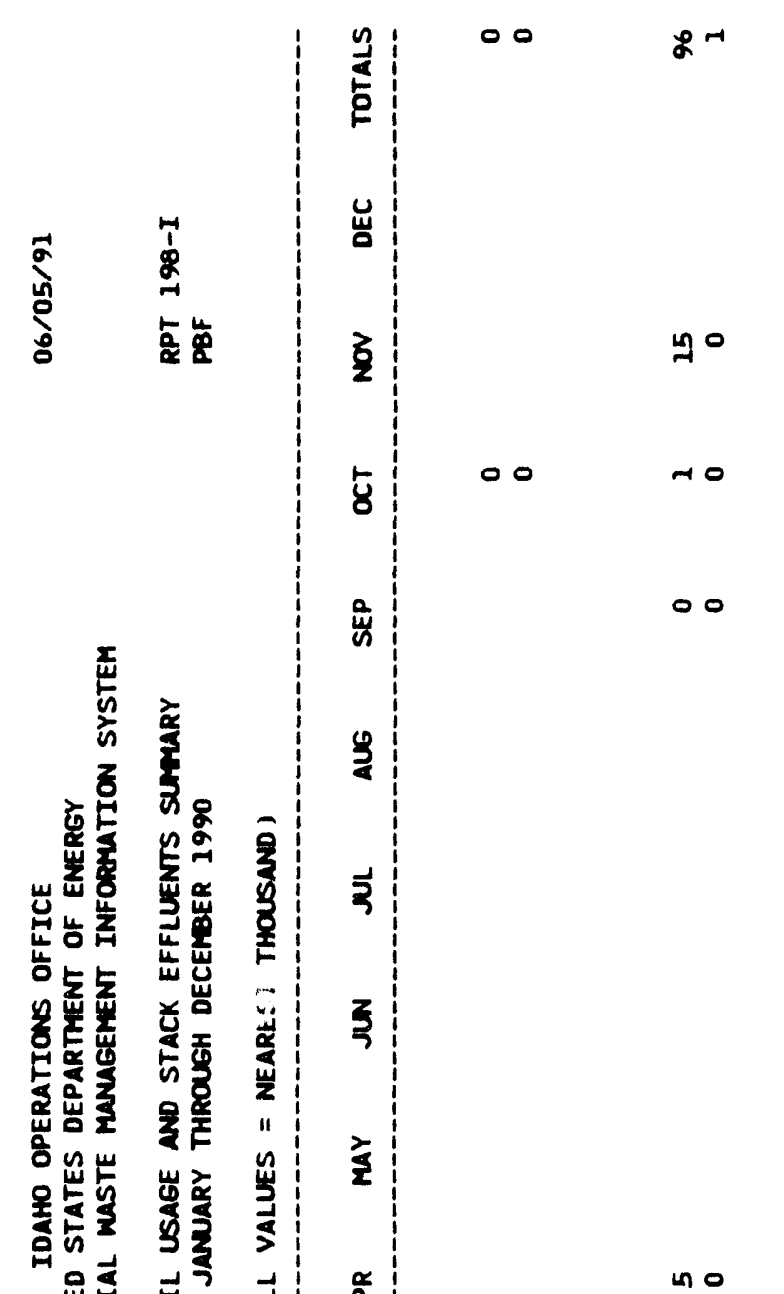

I

t

I

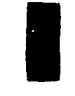

존

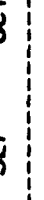

00

\#0

0

$\frac{3}{8}$

ร)

z

$\frac{2}{2}$

I

还表

$\frac{\alpha}{2}$

ino

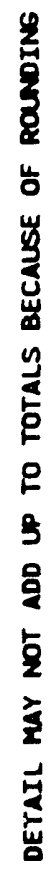

I

00

no

0

mo

0

3

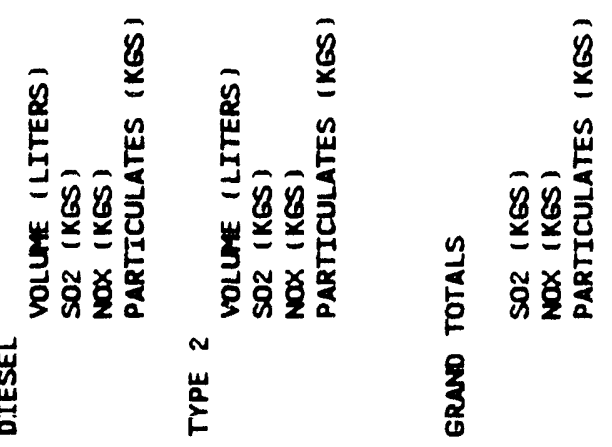

PBF-9 


\section{RADIOACTIVE WASTE MANAGEMENT COMPLEX, 1990 DETAIL DATA}

Report 256 - WMC Industrial Waste Summary $\ldots \ldots \ldots \ldots \ldots \ldots \ldots$ WMC-3

Report 257 - WMC Water Usage and Disposal Summany . . . . . . . . . WMC-4 


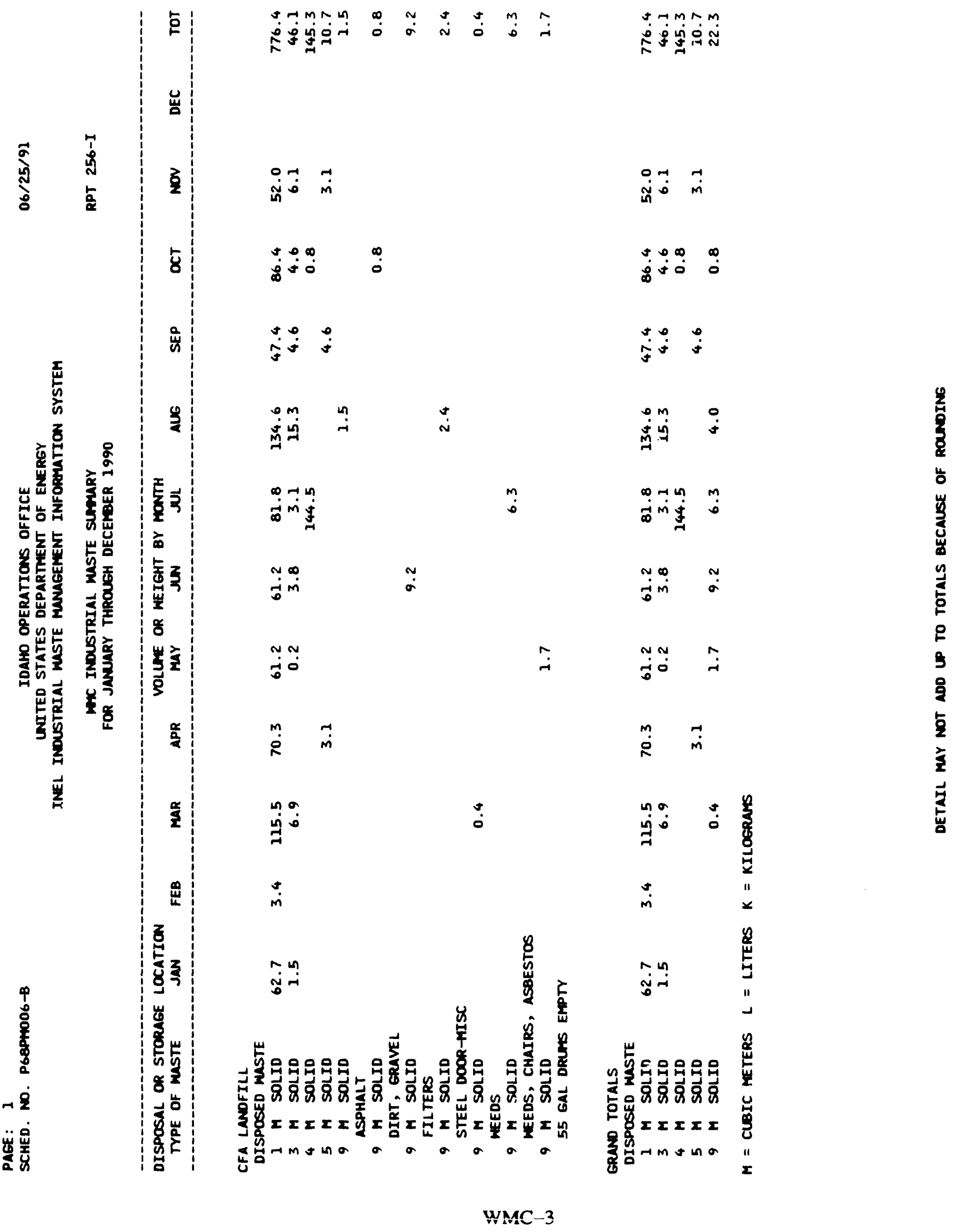




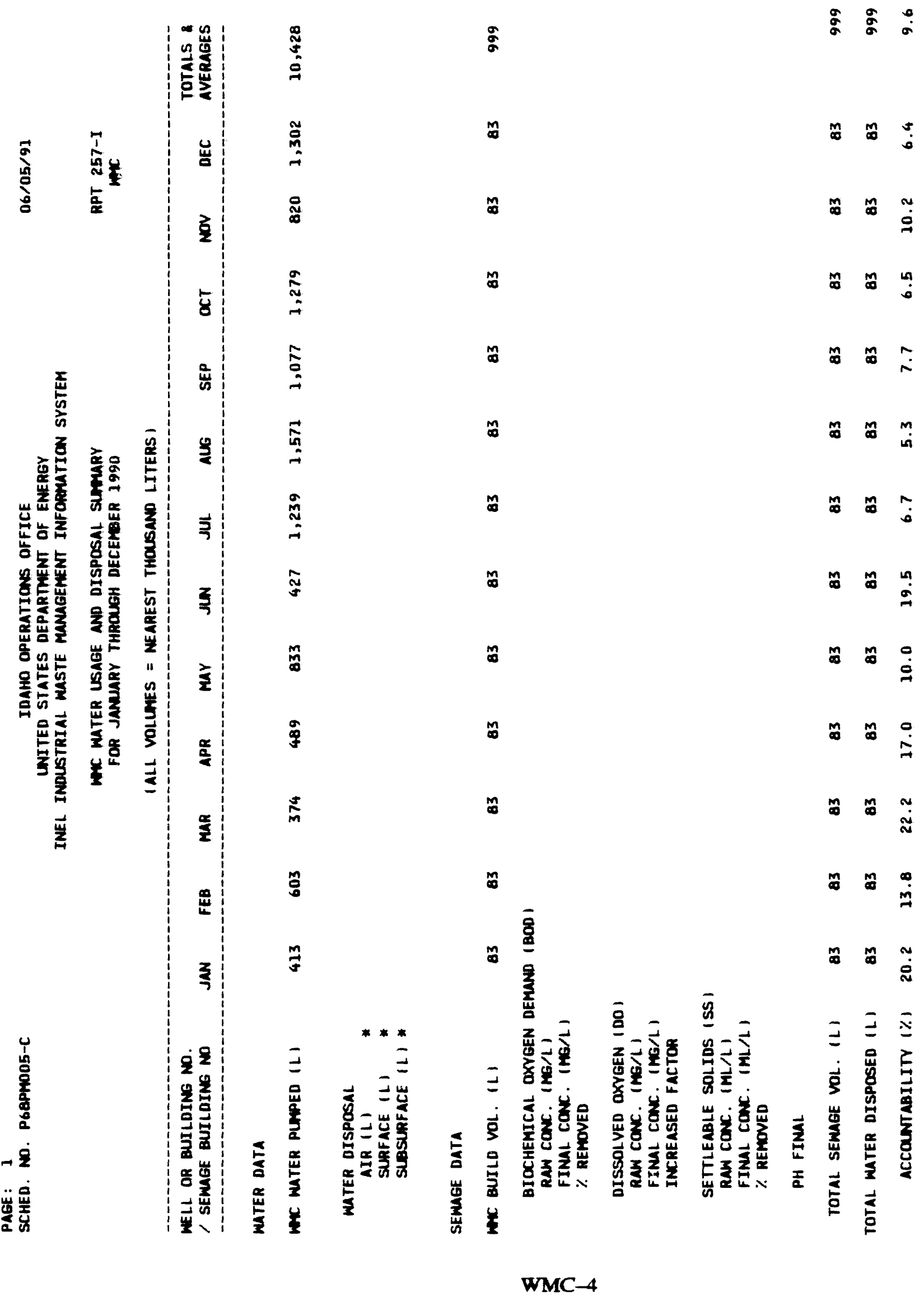




\section{TEST REACTOR AREA, 1990 DETAIL. DATA}

Report 234 - TRA Airborne Disposed Substances Summary ........... TRA-3

Report 235 - TRA Liquid Disposed Substances Summary . . . . . . . . . TRA-4

Report 236 - TRA Industrial Waste Summary $\ldots \ldots \ldots \ldots \ldots \ldots \ldots$ TRA 8

Report 237 - TRA Water Usage and Disposal Summary . . . . . . . . . . TRA-9

Report 238 - TRA Fiel Oil Usage and Stack Effluents Summary . . . . . . . . TRA-10 


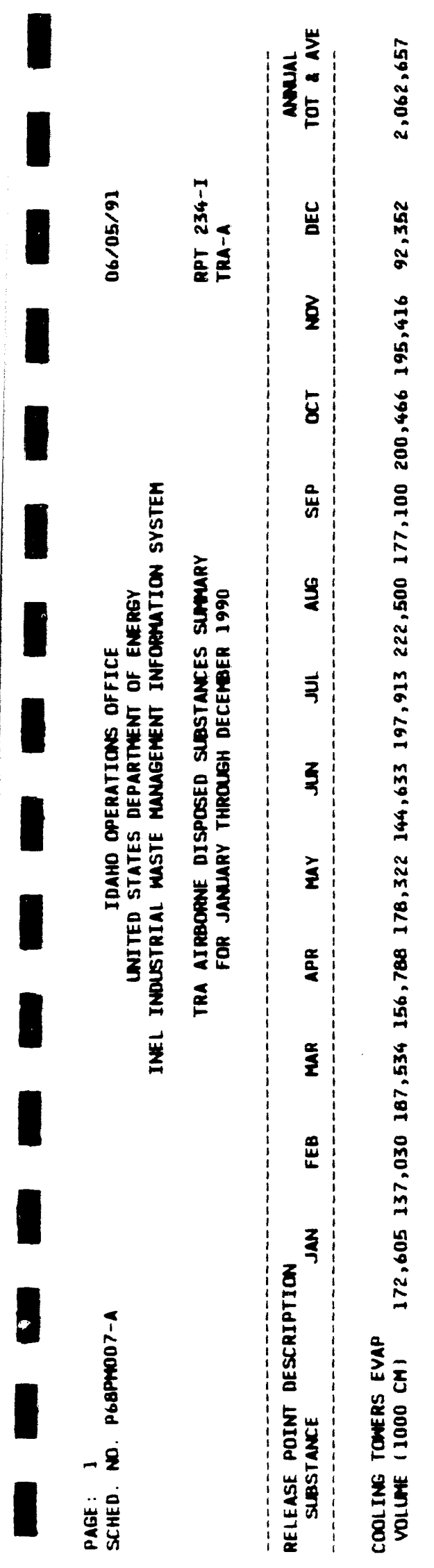




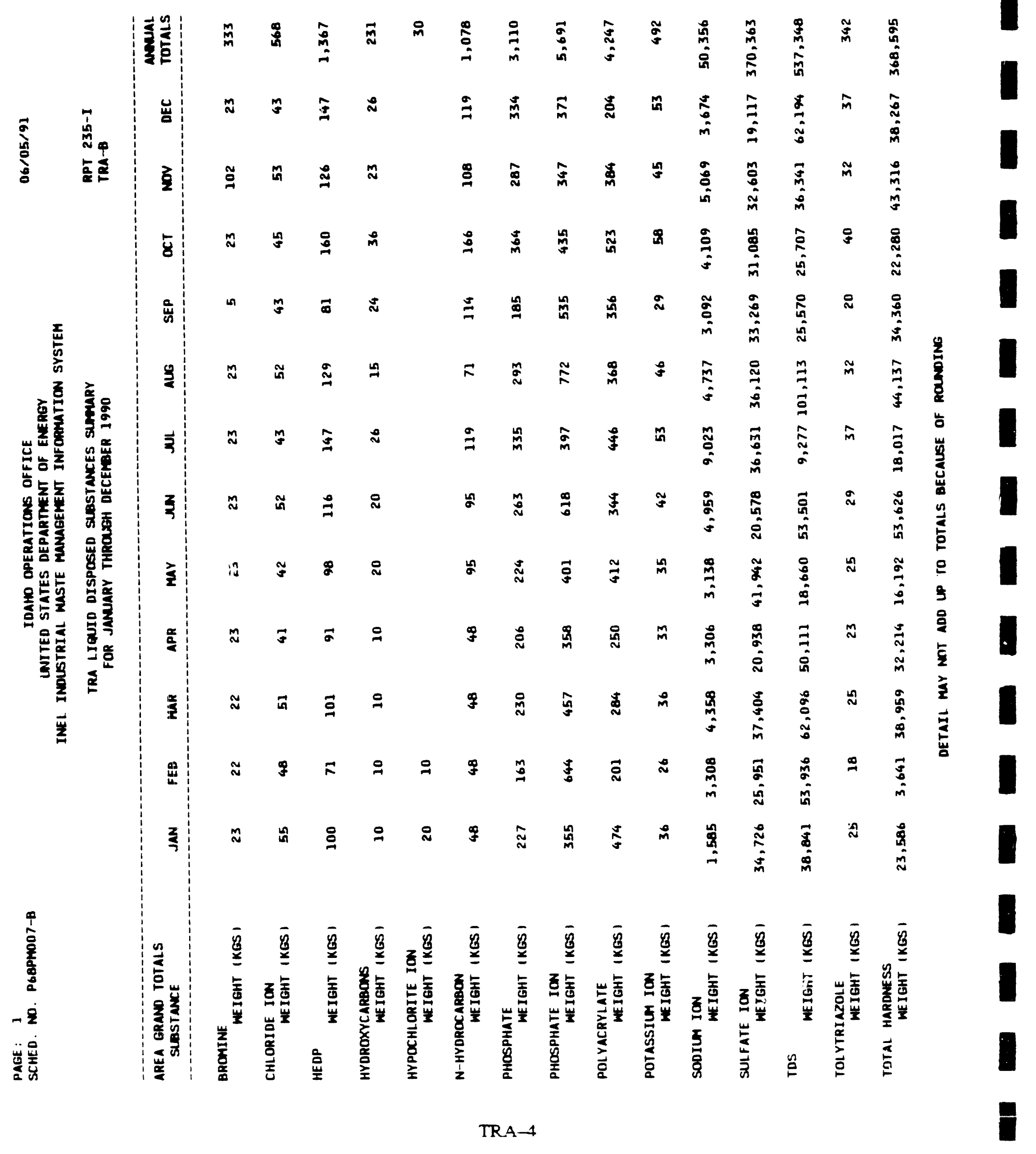




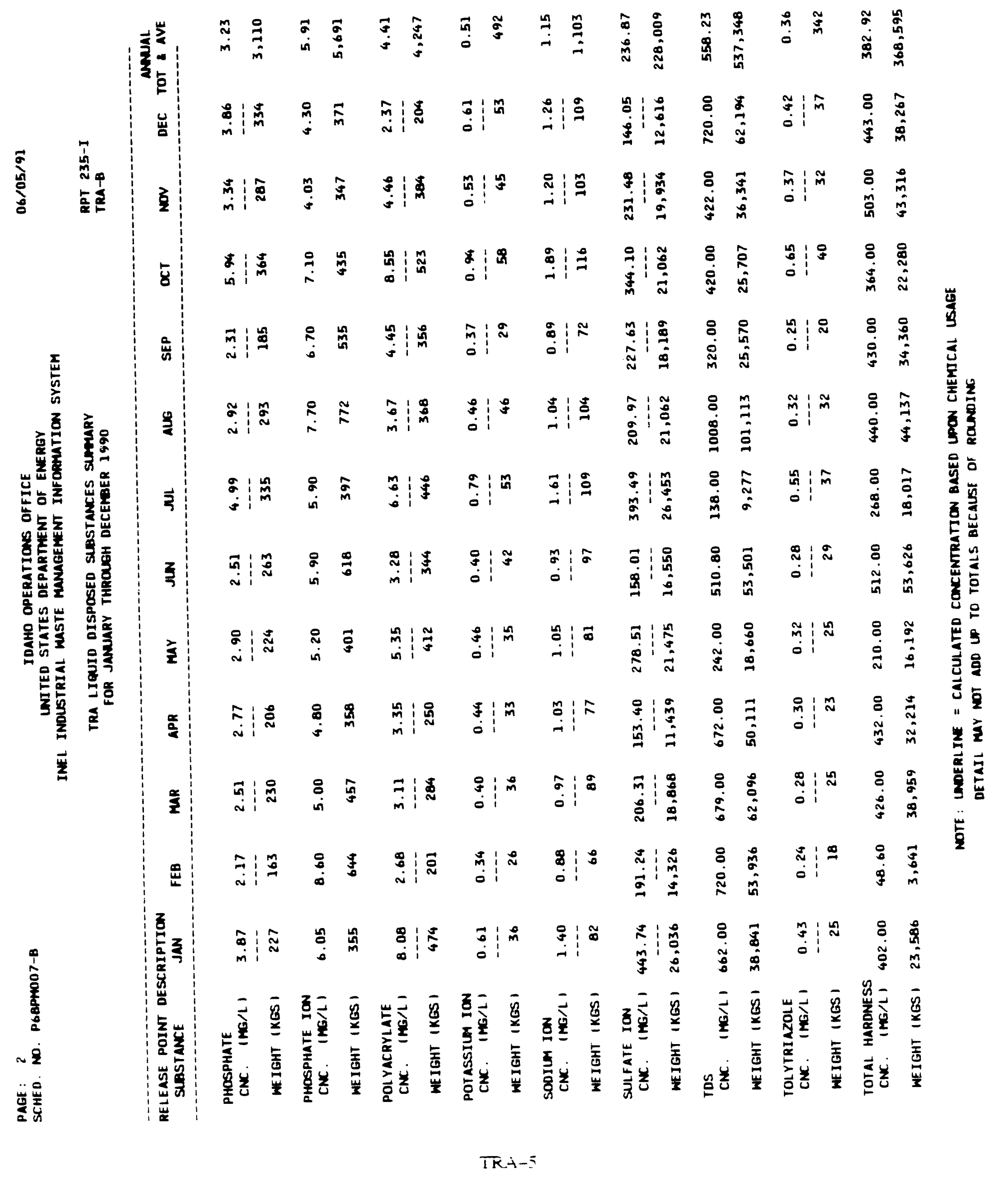




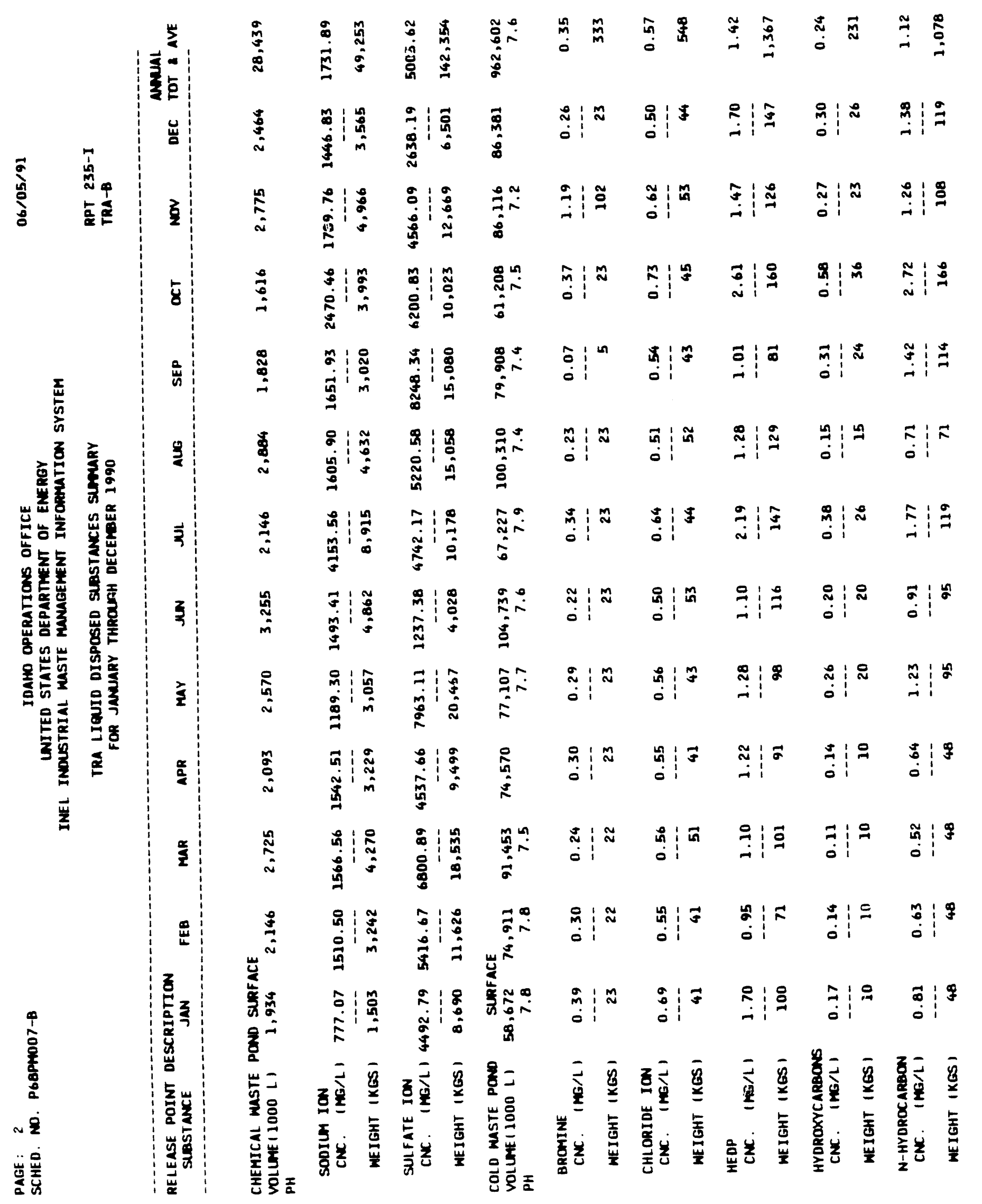

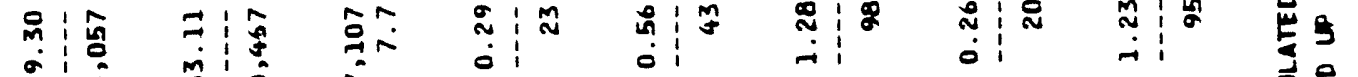




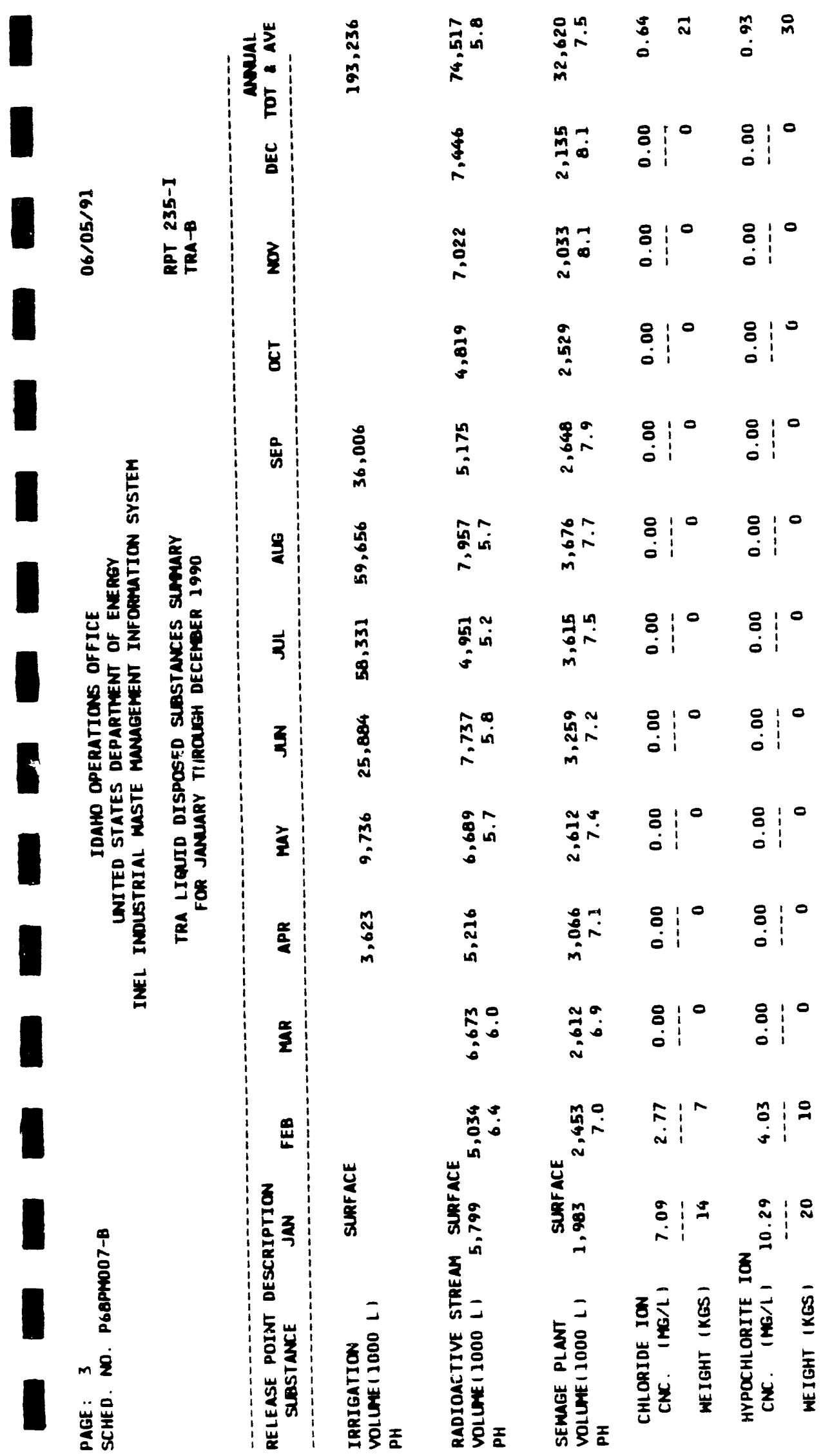




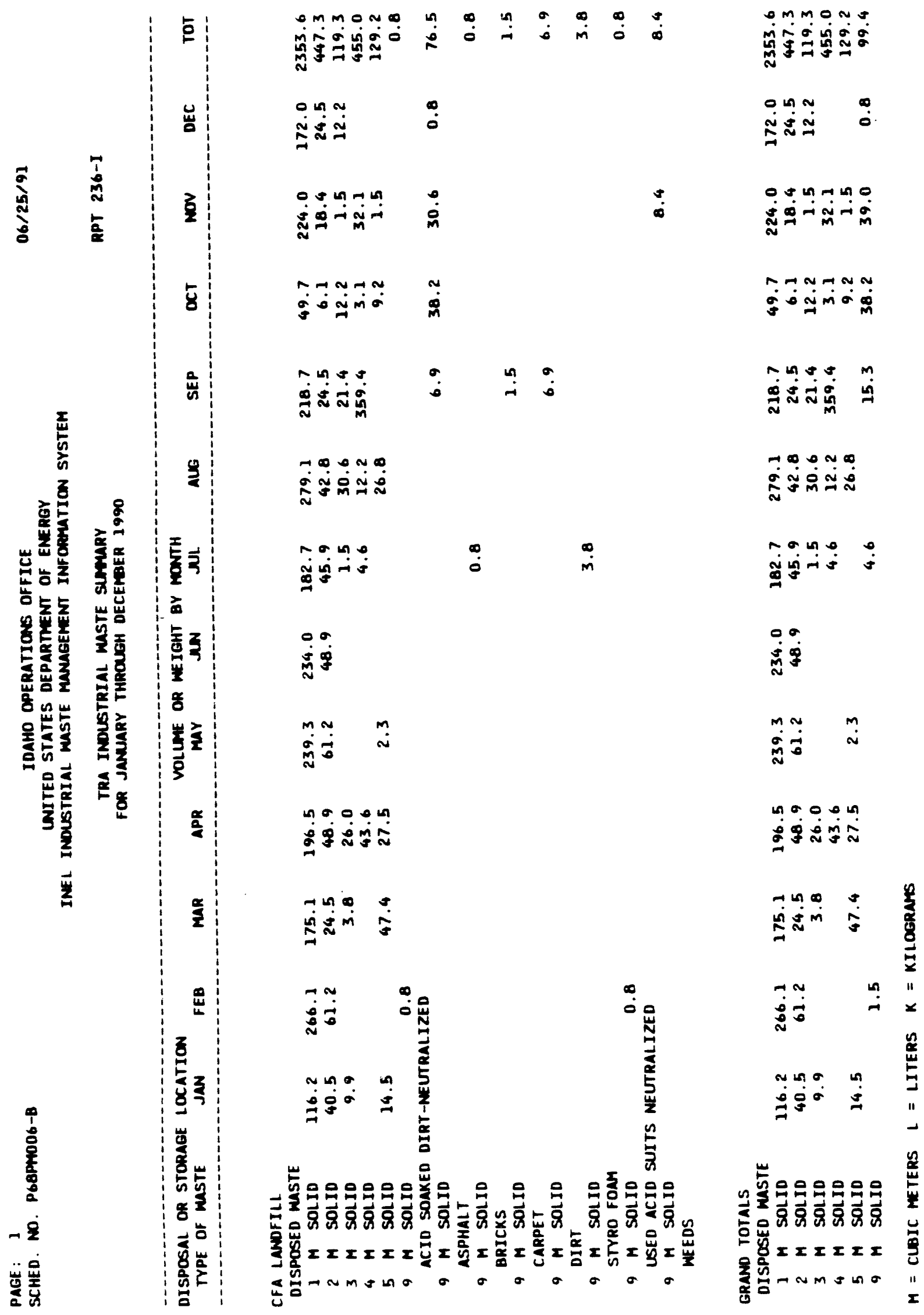




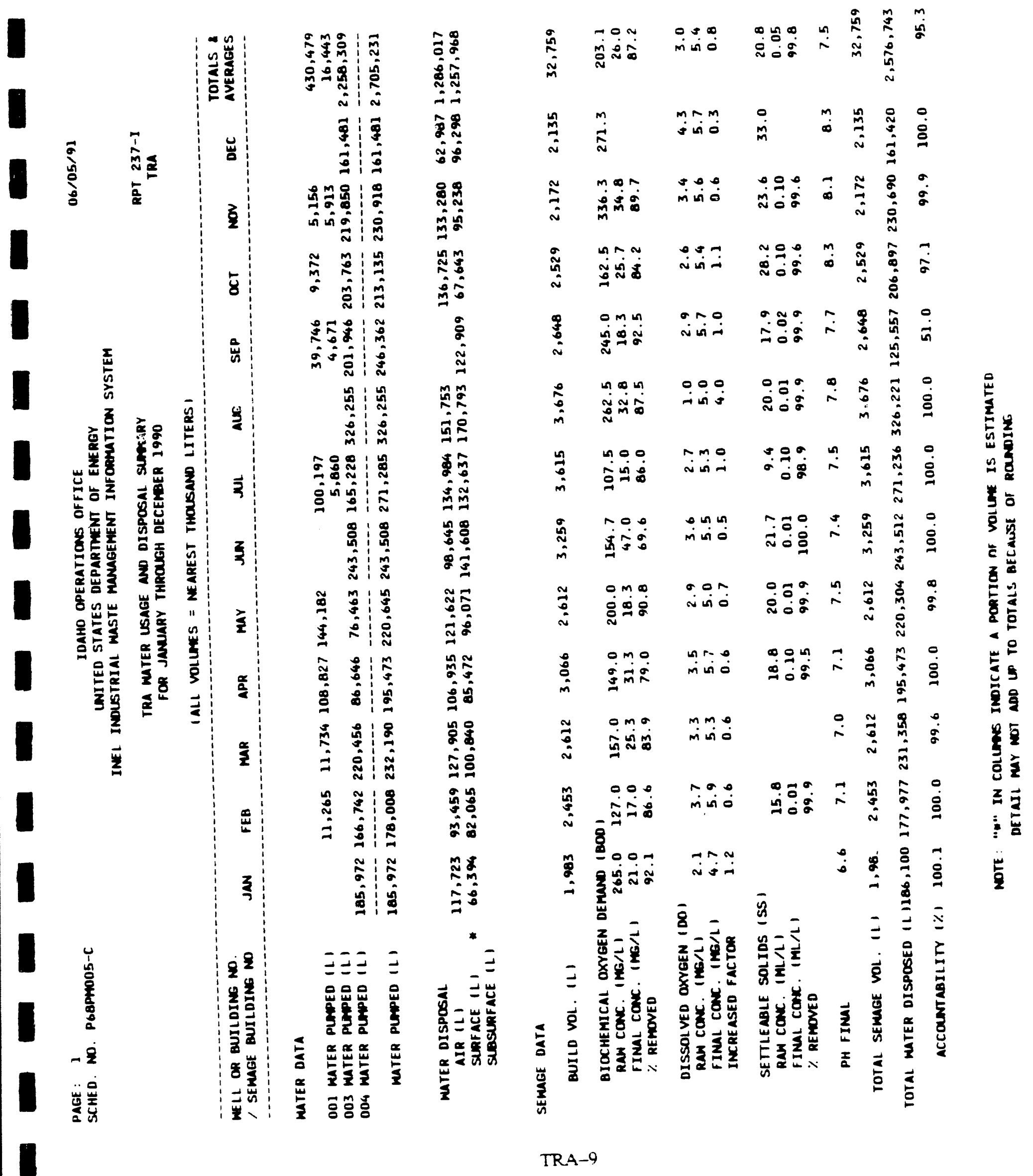




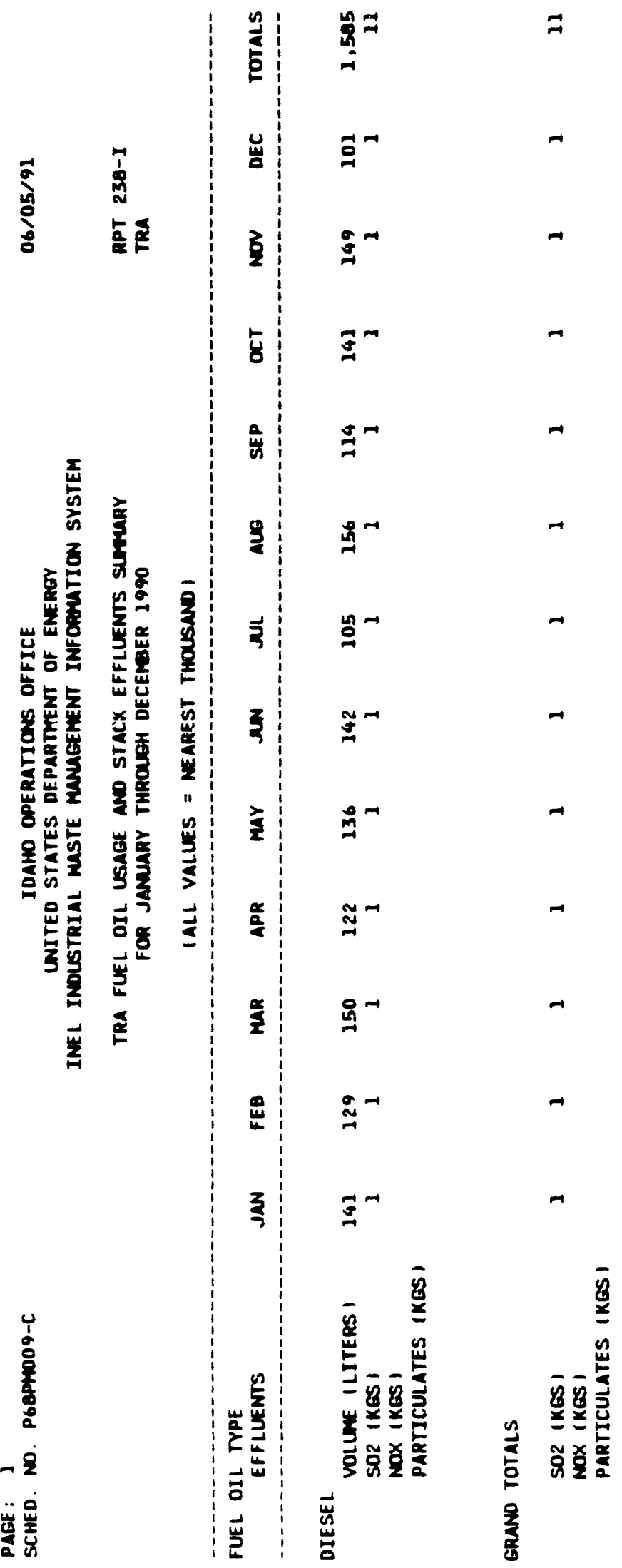


TECHNICAL SUPPORT FACILITY - TEST AREA NORTH, 1990 DETAIL DATA

Report 225 - TSF Liquid Disposed Substances Summary $\ldots \ldots \ldots \ldots \ldots$ TSF-3

Report 226 - TSF Industrial Waste Summary $\ldots \ldots \ldots \ldots \ldots \ldots \ldots$ TSF -6

Report 227 - TSF Water Usage and Disposal Summary . . . . . . . . . . TSF-7

Report 228 - TSF Fuel Oil Usage and Stack Effluents Summary . . . . . . . . TSF-8 


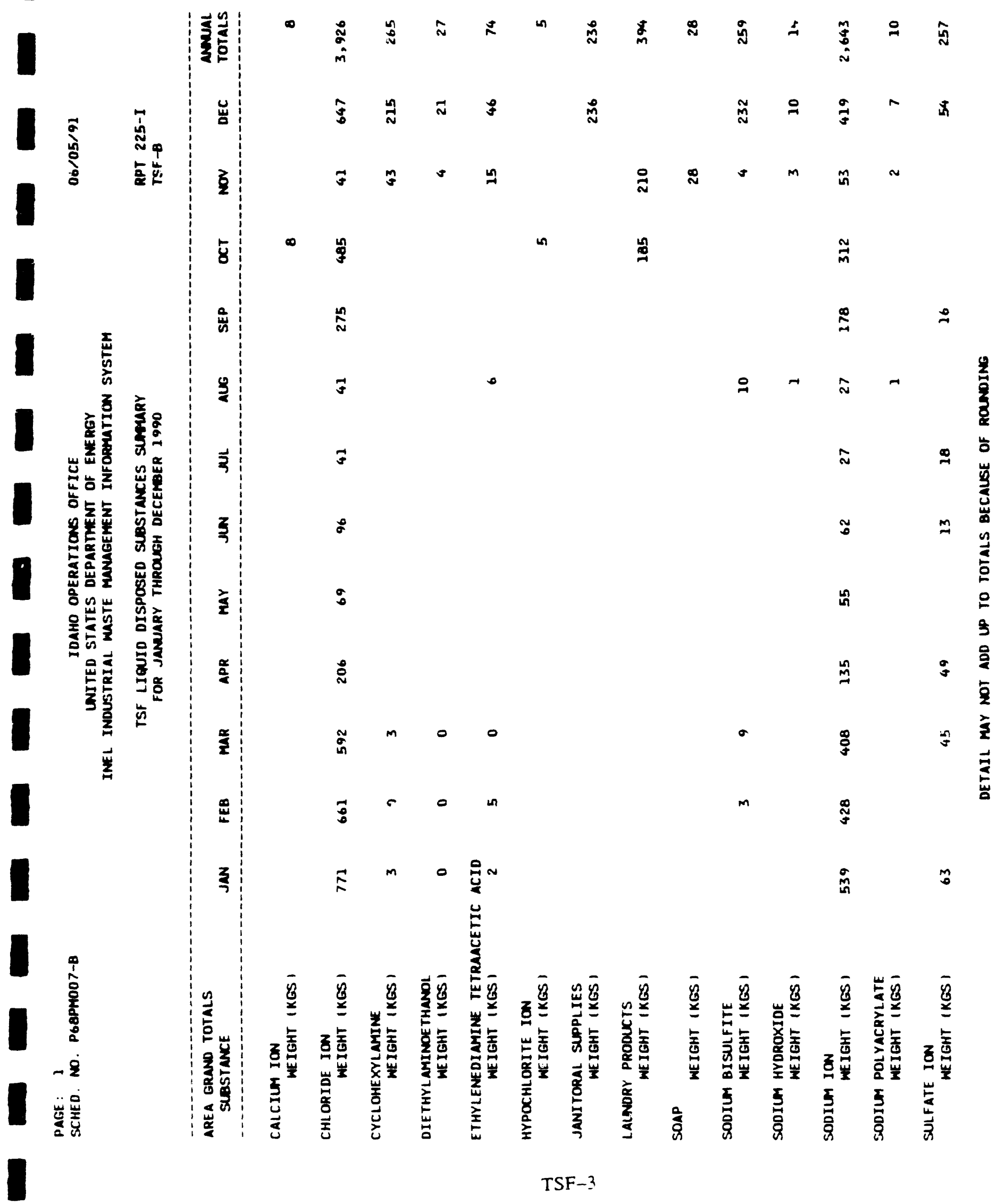




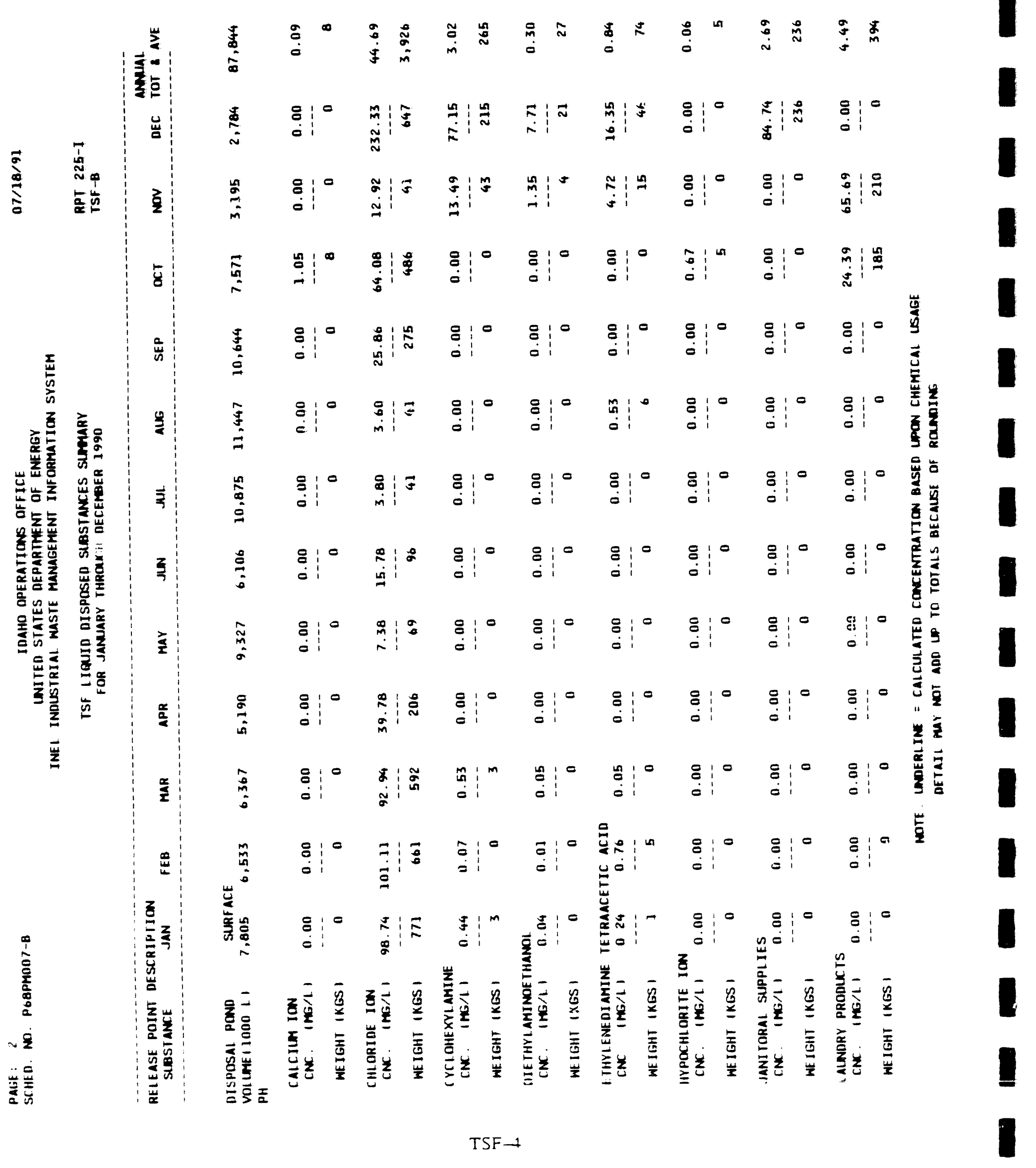


I

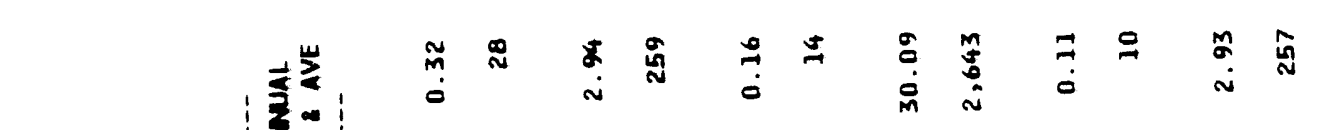

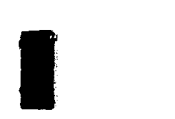

占

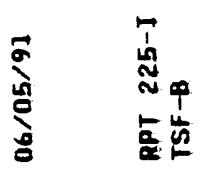

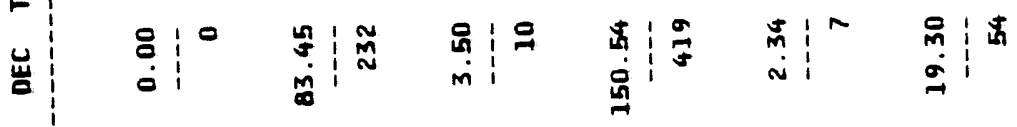

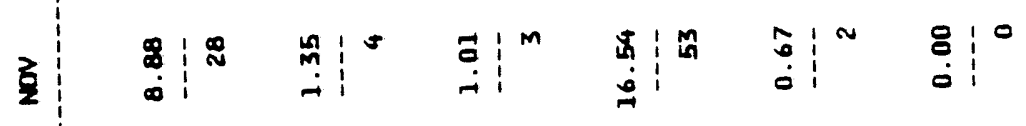

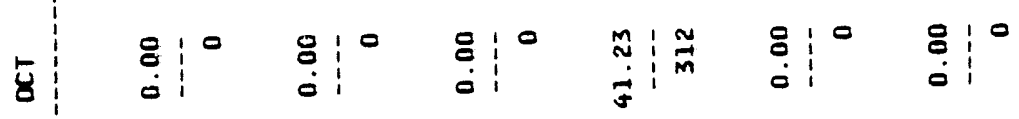

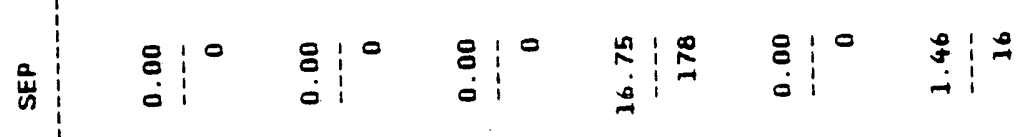

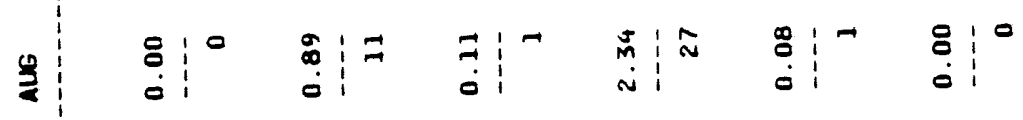

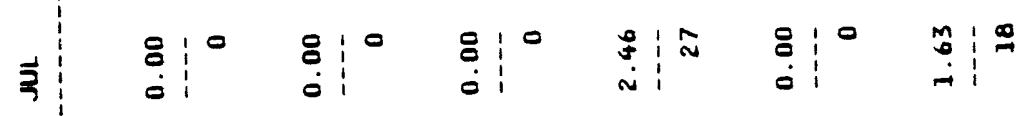

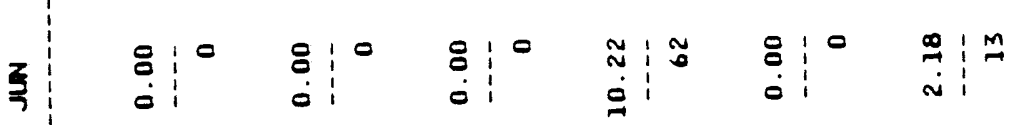

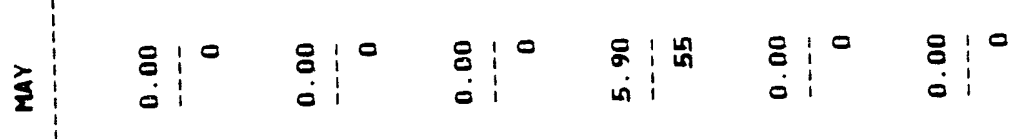

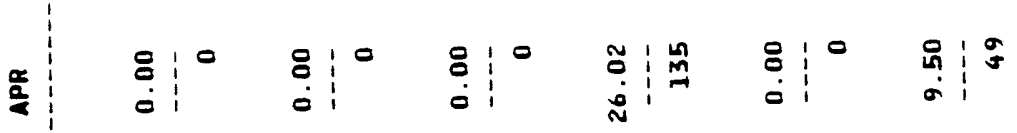

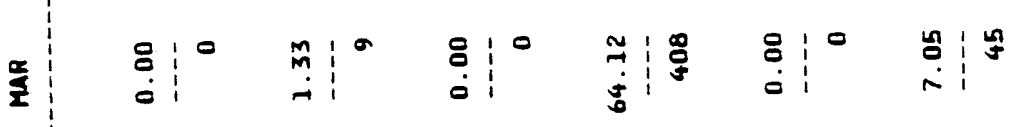

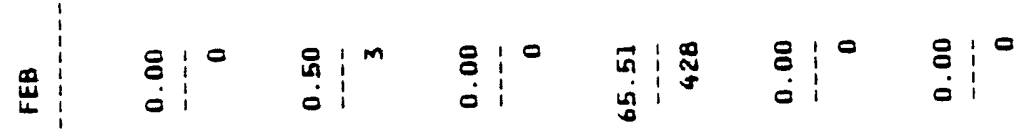

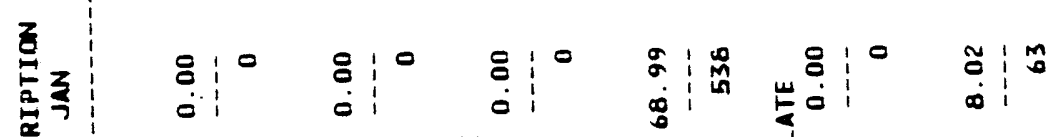

紊

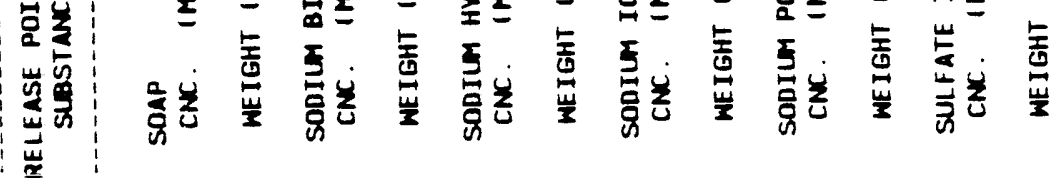

$\sim z$

蒙蜜 

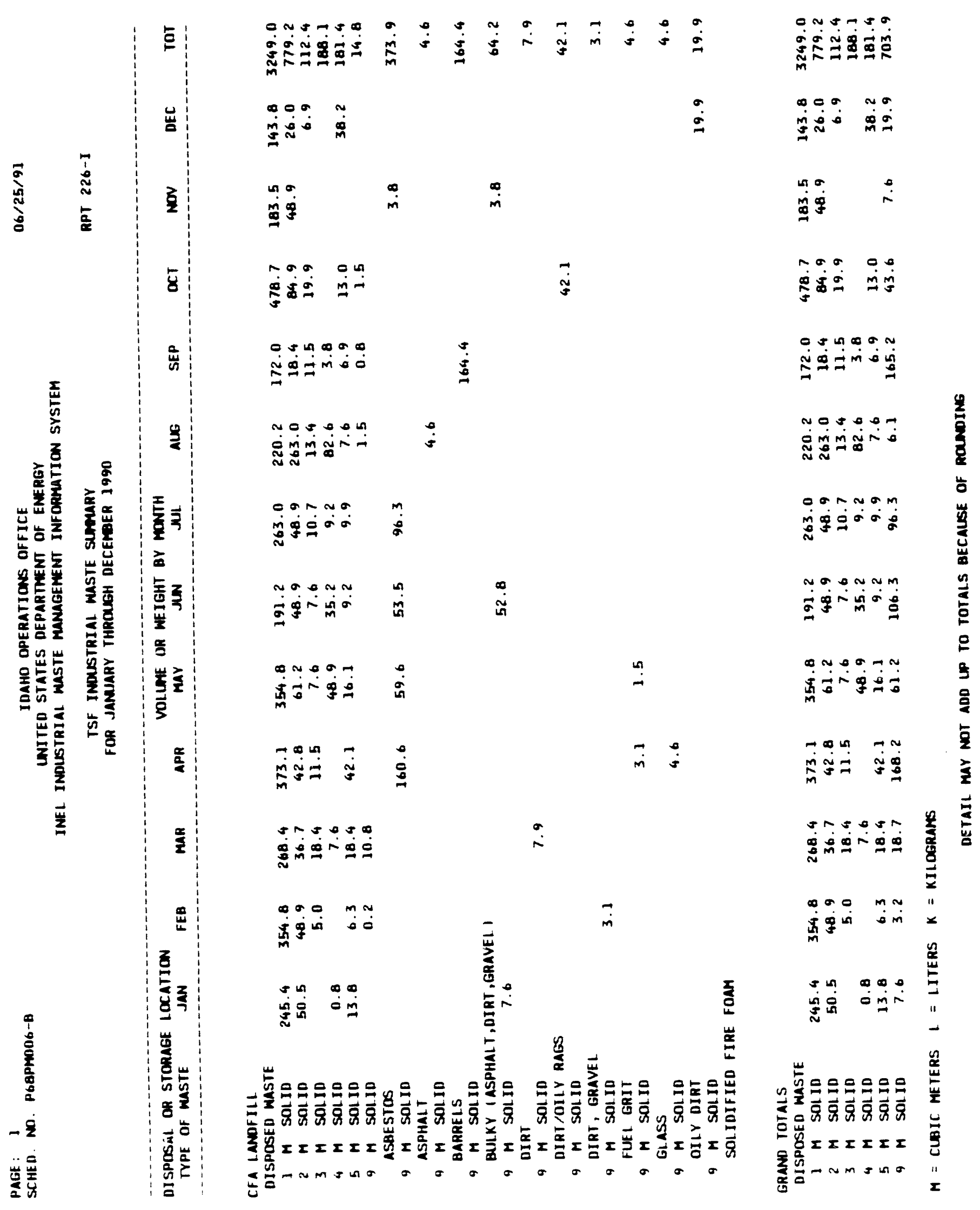

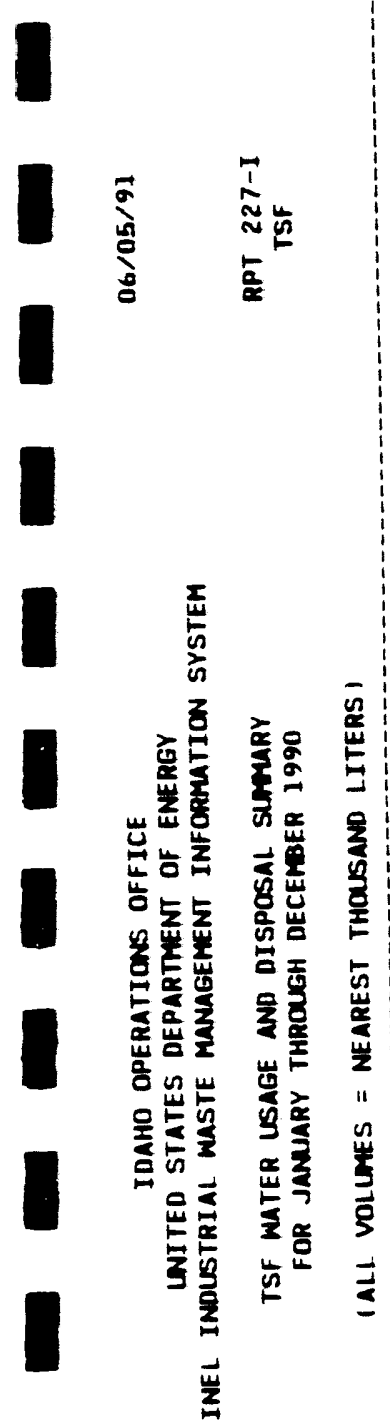

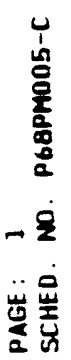

|⿱⺈⿻コ一心

$\ddot{\square}$

z

8

㟧

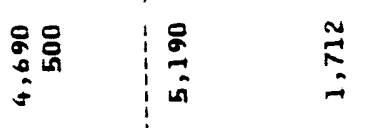

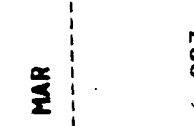

这

3

要

骂薪

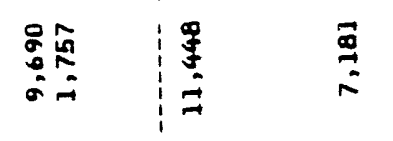

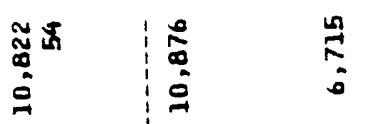

\begin{tabular}{l|l}
0 & 0 \\
\hdashline & 0
\end{tabular}

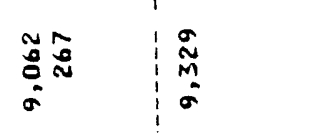

鮊

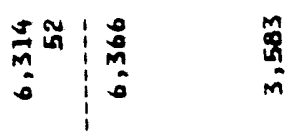

萬然

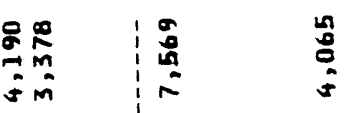

$\stackrel{5}{\circ}$

$\stackrel{0}{=}$

i

$\stackrel{n}{\hat{a}}$

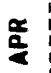

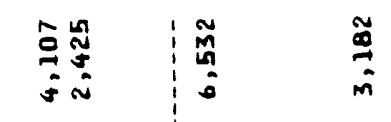

ind

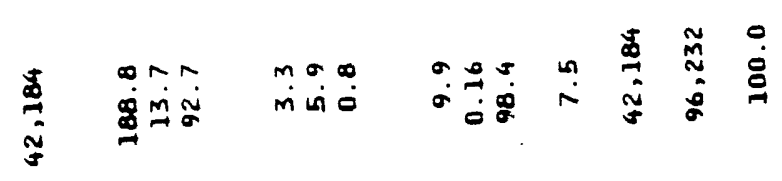

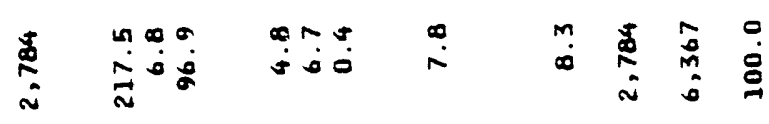

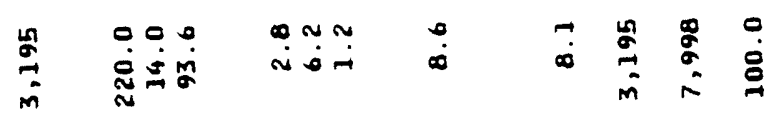

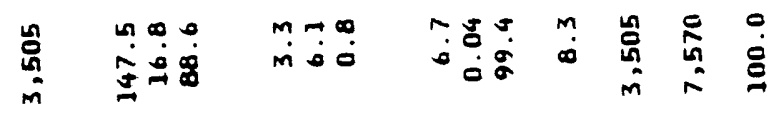

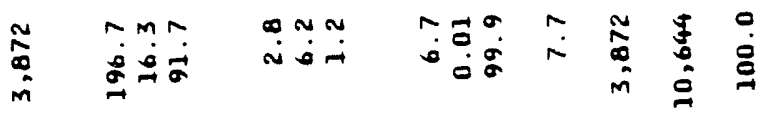

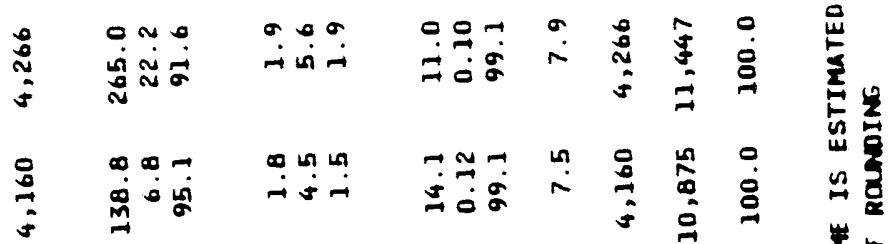

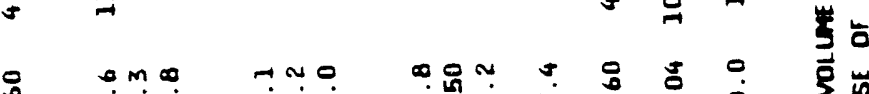

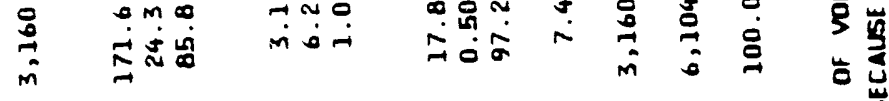

萑

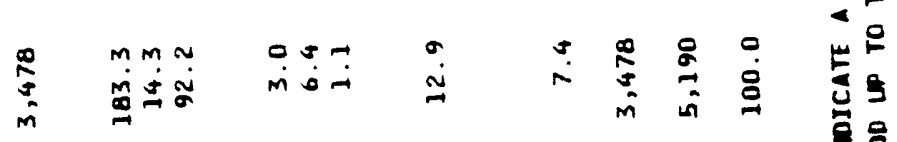

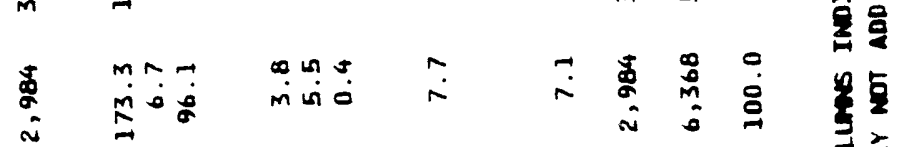

mog on o 0 a

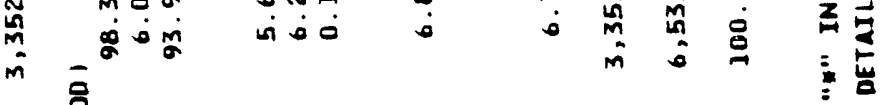

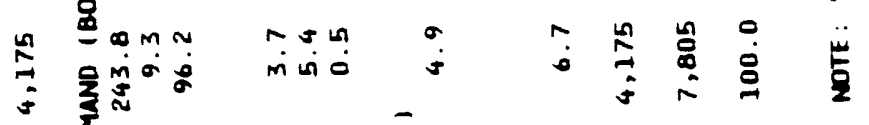

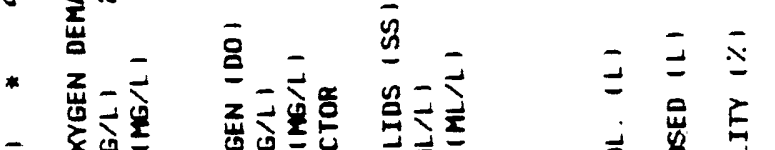

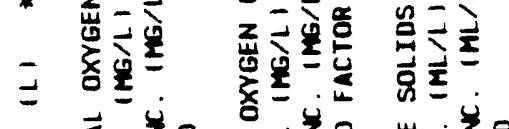

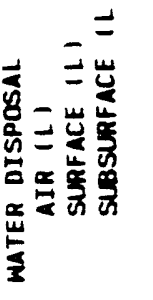

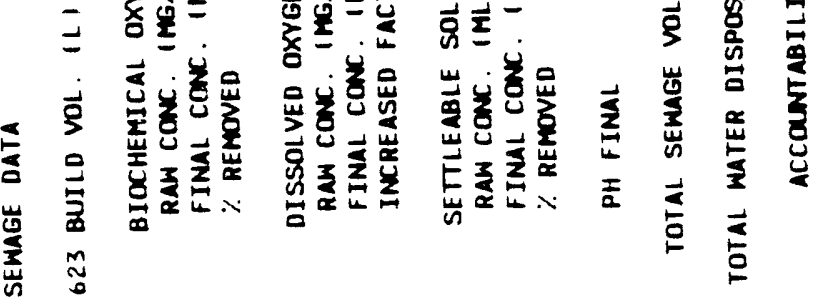




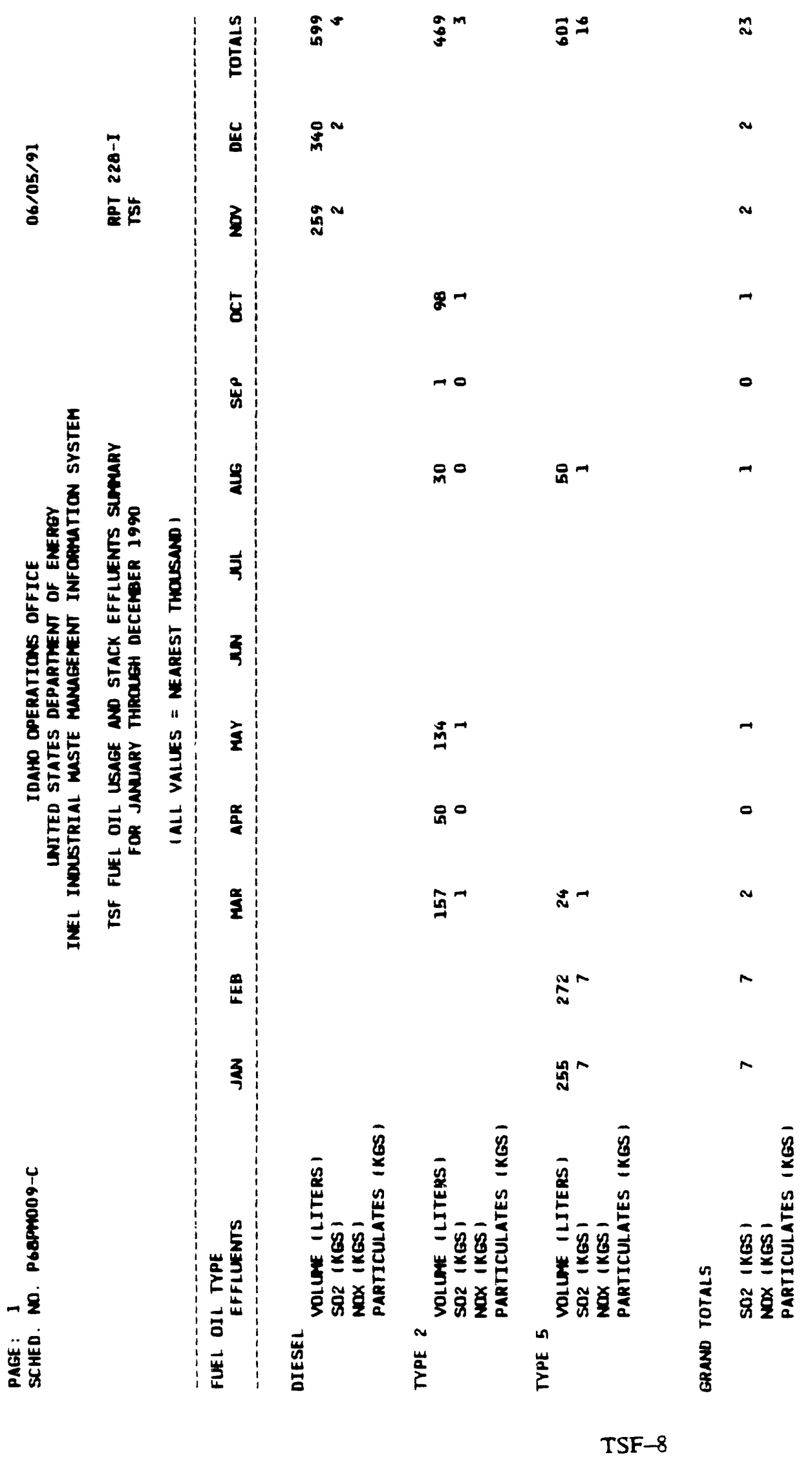


WATER REACTOR RESEARCH TEST FACILITY - TEST AREA NORTH, 1990 DETAIL DATA

Report 265 - WRF Liquid Disposed Substances Summary ............ WRF-3

Report 267 - WRF Water Usage and Disposal Summary . . . . . . . . W WRF-5

Report 268 - WRF Fuel Oil Usage and Stack Effluents Summary . . . . . . . W WRF-6 


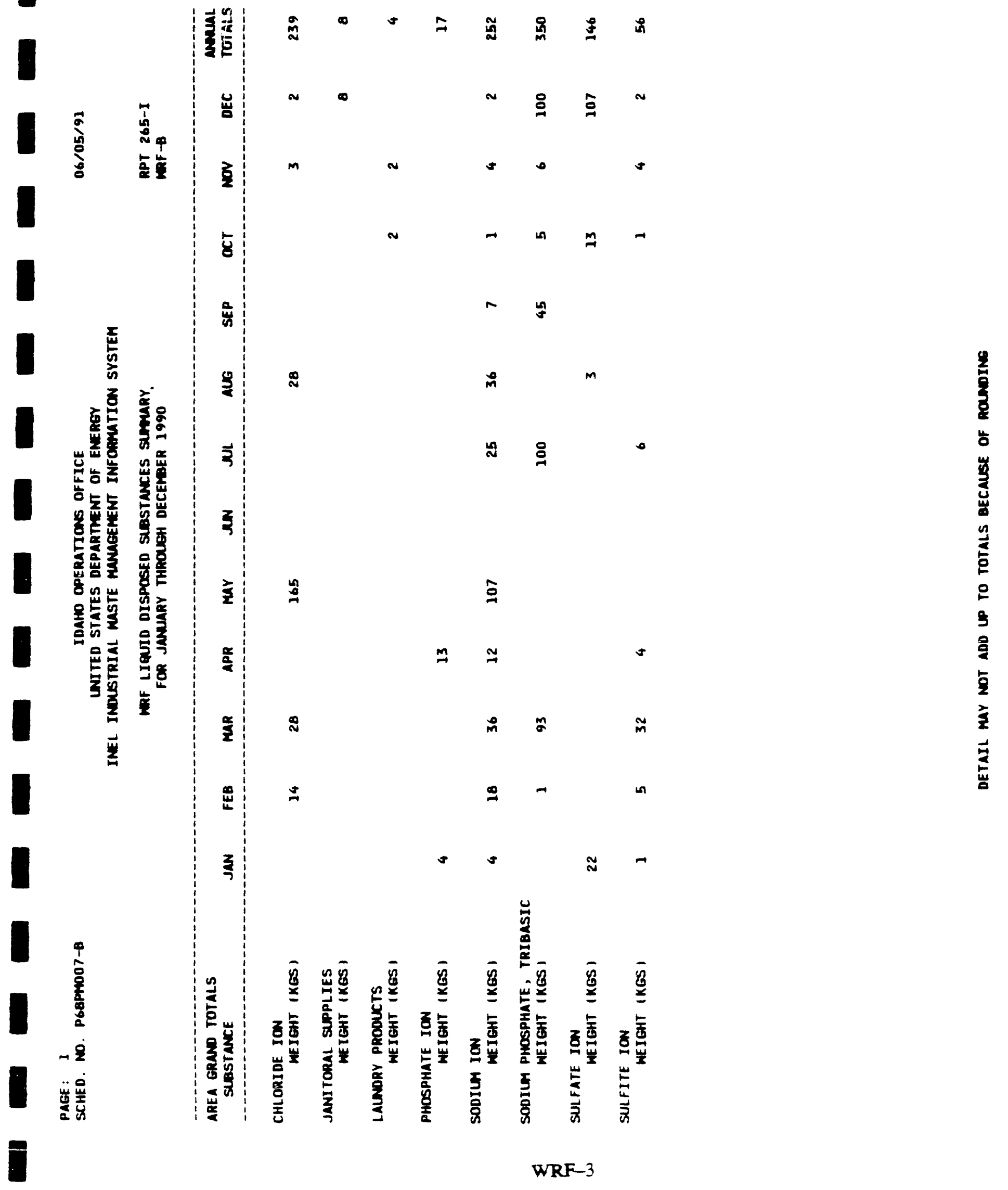




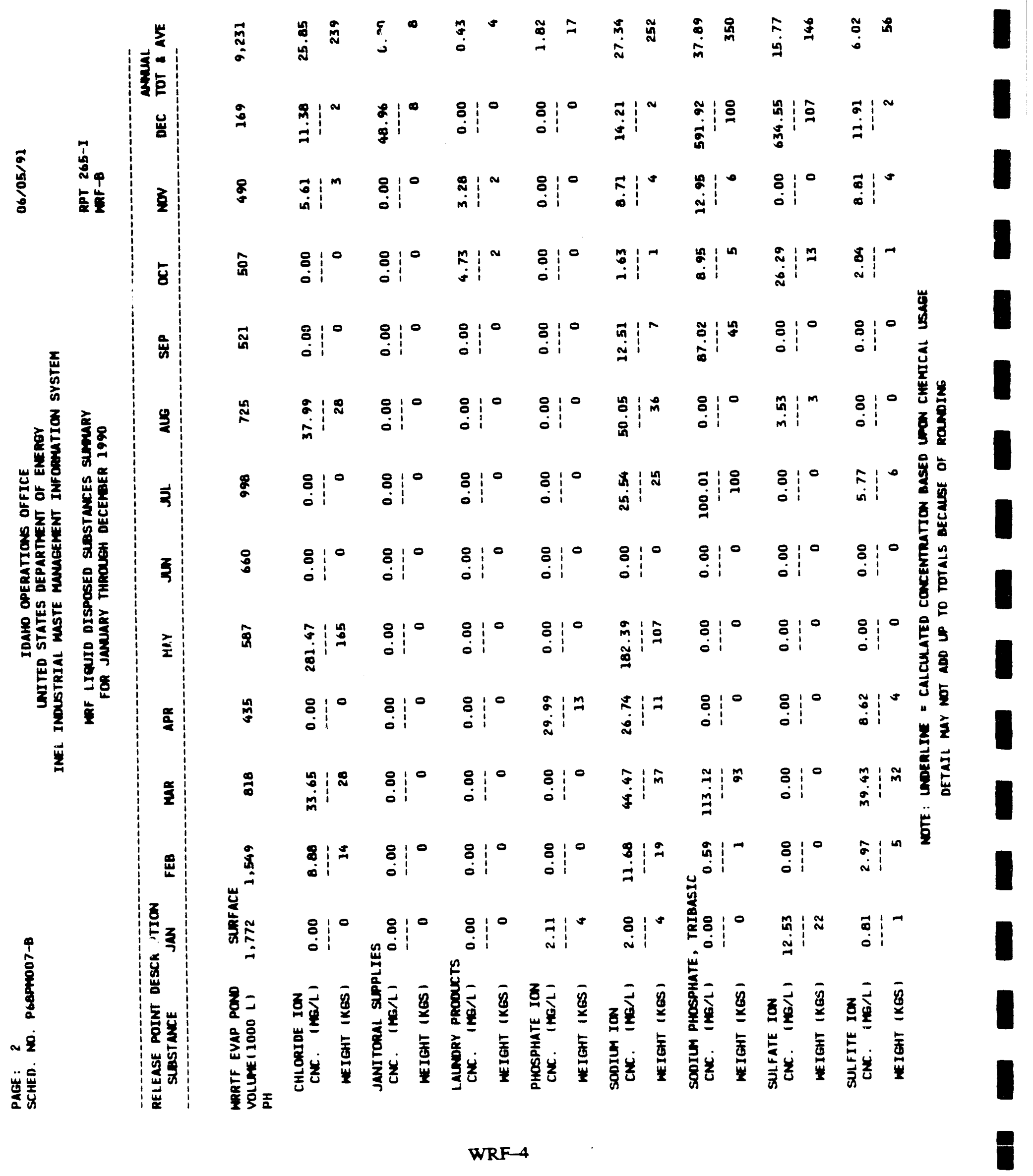




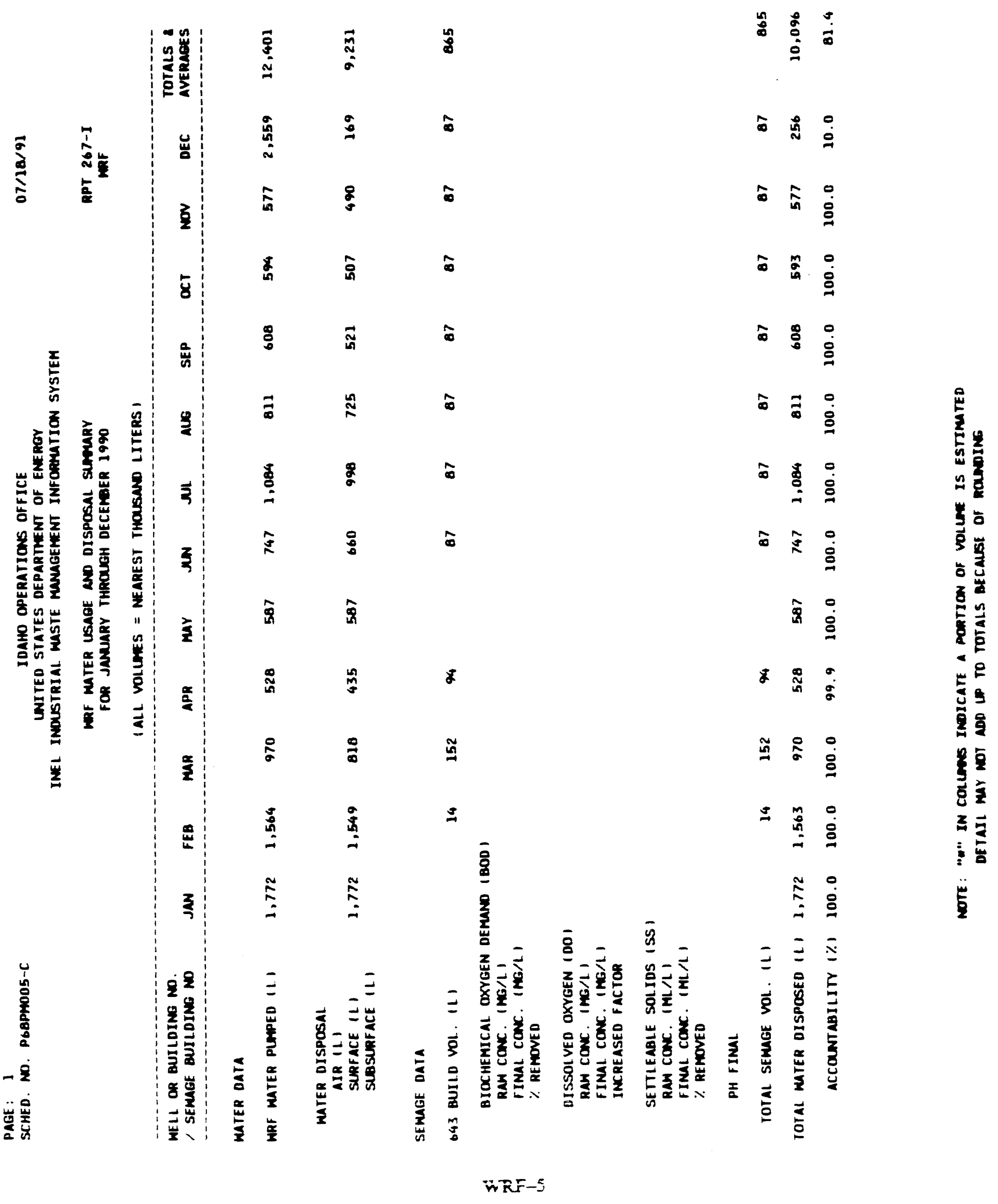




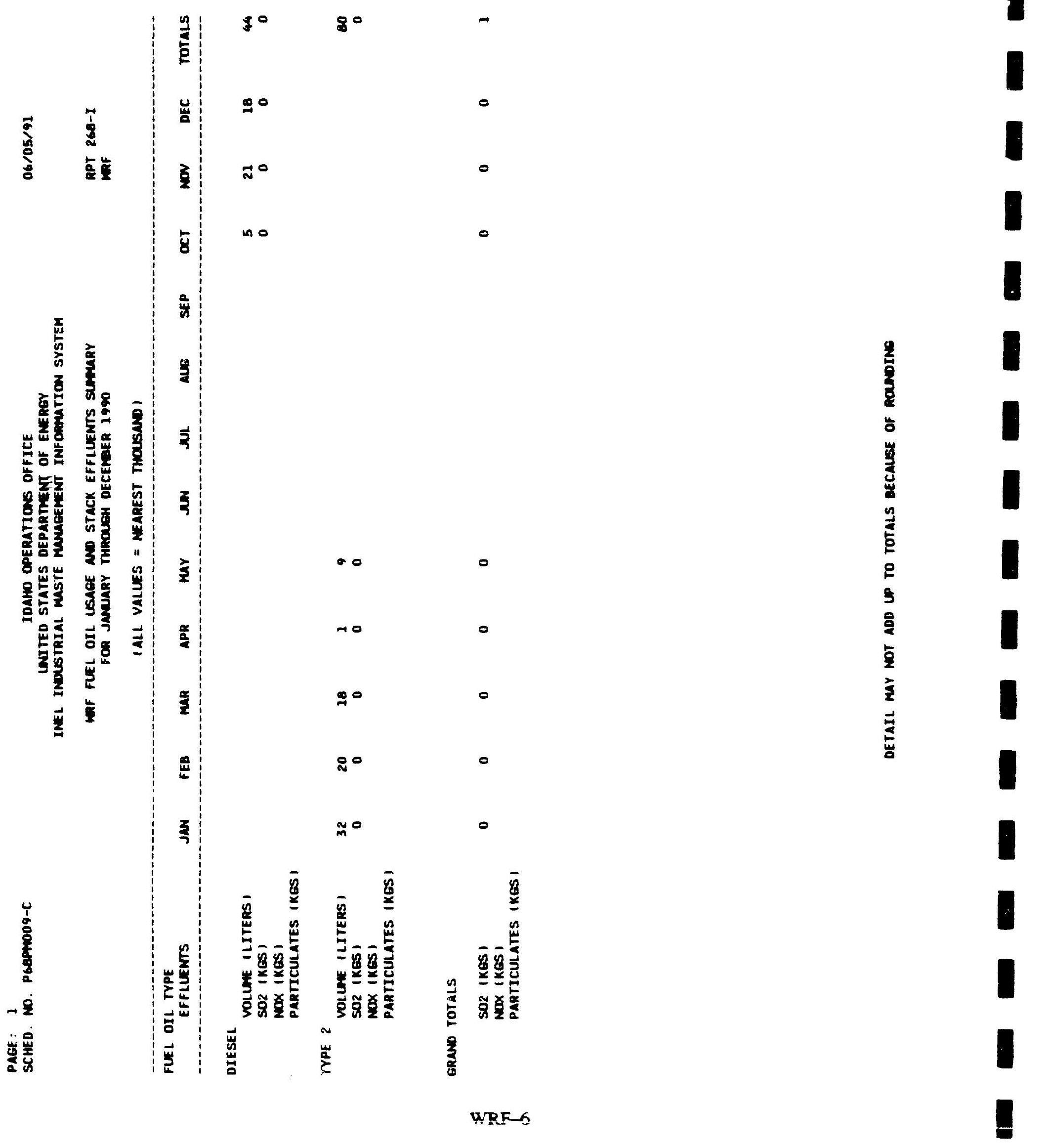



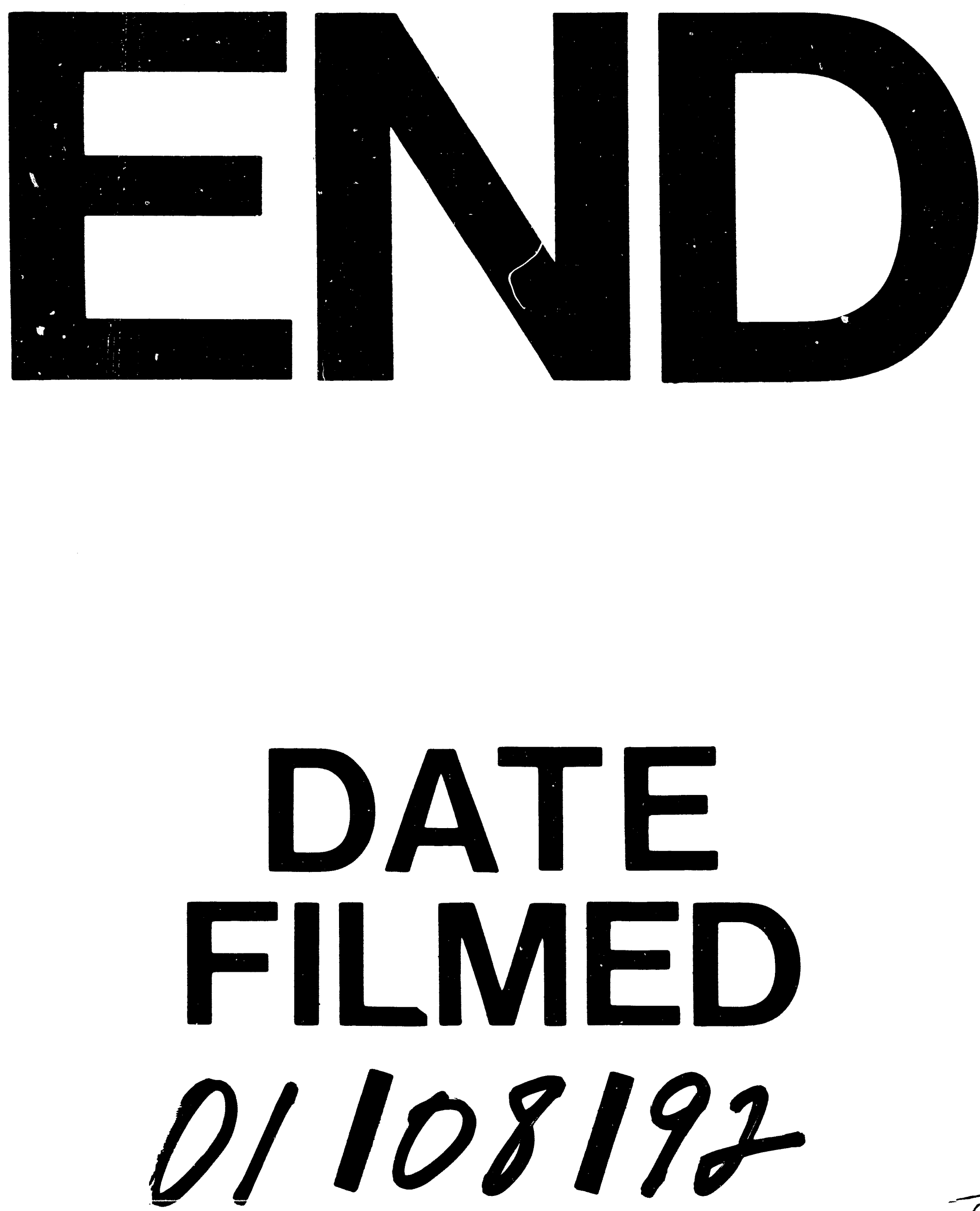
